\title{
The SRSF1/circATP5B/miR-185-5p/HOXB5 Feedback Loop Regulates the Proliferation of Glioma Stem Cells via the IL6-mediated JAK2/STAT3 Signaling Pathway
}

Junshuang Zhao

The First Affiliated Hospital of China Medical University

Yang Jiang

The First Affiliated Hospital of China Medical University

Haiying Zhang

Liaoning University of Traditional Chinese Medicine Jinpeng Zhou

The First Affiliated Hospital of China Medical University Lian Chen

The first affiliated hospital of China Medical University

Hao Li

The first affiliated hospital of China Medical University

Jinkun Xu

The first affiliated hospital of China Medical University

\section{Guoqing Zhang}

The first affiliated hospital of China Medical University

\section{Zhitao Jing ( $\nabla_{\text {jingzhitao@hotmail.com ) }}$}

China Medical University Hospital

\section{Research}

Keywords: Glioma stem cells, circATP5B, miR-185-5P, HOXB5, SRSF1, IL6, JAK2/STAT3 signaling

Posted Date: December 15th, 2020

DOl: https://doi.org/10.21203/rs.3.rs-125946/v1

License: (1) This work is licensed under a Creative Commons Attribution 4.0 International License. Read Full License 
Version of Record: A version of this preprint was published at Journal of Experimental \& Clinical Cancer Research on April 15th, 2021. See the published version at https://doi.org/10.1186/s13046-021-01931-9. 


\section{Abstract}

\section{Background}

Glioma is the most common and malignant tumor of central nervous system. The tumor initiation, selfrenewal, and multi-lineage differentiation abilities of glioma stem cells (GSCs) are responsible for glioma proliferation and recurrence. Although circular RNAs (circRNAs) play vital roles in the progression of glioma, the detailed mechanisms remain unknown.

\section{Methods}

qRT-PCR, western blotting, immunohistochemistry, and bioinformatic analysis were performed to detect the expression of circATP5B, miR-185-5p, HOXB5, and SRSF1. Patient-derived GSCs were established, and MTS, EDU, neurosphere formation, and limiting dilution assays were used to detect the proliferation of GSCs. RNA-binding protein immunoprecipitation, RNA pull-down, luciferase reporter assays, and chromatin immunoprecipitation assays were used to detect these molecules' regulation mechanisms.

Results

We found circATP5B expression was significantly upregulated in GSCs and promoted the proliferation of GSCs. Mechanistically, circATP5B acted as miR-185-5p sponge to upregulate HOXB5 expression. HOXB5 was overexpressed in glioma and transcriptionally regulated IL6 expression and promoted the proliferation of GSCs via JAK2/STAT3 signaling. Moreover, RNA binding protein SRSF1 could bind to and promote circATP5B expression and regulate the proliferation of GSCs, while HOXB5 also transcriptionally regulated SRSF1 expression.

\section{Conclusions}

Our study identified the SRSF1/circATP5B/miR-185-5p/HOXB5 feedback loop in GSCs. This provides an effective biomarker for glioma diagnosis and prognostic evaluation.

\section{Background}

Glioma is the most common and malignant tumor among central nervous system cancers and is associated with poor prognosis in patients $(1,2)$. Despite advances in surgery, radiotherapy, and chemotherapy, treatment outcomes in glioma patients remain poor, with an average survival time of approximately 15 months (3). Glioma stem cells (GSCs) have been reported to be responsible for glioma and glioblastoma proliferation, therapeutic resistance, and recurrence due to their abilities in tumor initiation, self-renewal, and multi-lineage differentiation (4). Therefore, studying the mechanisms of GSCs may provide important insights into potential strategies for glioblastoma therapy.

Circular RNA (circRNA) is an endogenous non-coding RNA mainly composed of exons and (or) introns that form a closed-loop structure through back splice sites between the $5^{\prime}$ and $3^{\prime}$ ends $(5,6)$. CircRNAs are 
more stable and higher in abundance in different tissues and cells than linear RNAs (7). Recently, circRNAs were proven to be involved in various pathological processes in cancer, such as cell proliferation, migration, invasion, apoptosis, and autophagy (8-12). CircRNAs operate via several functional mechanisms, including miRNA sponging, binding to RNA-binding proteins, modulating translation, and miRNA sponging is the most common role of circRNAs in the development of tumors $(13-15)$. Several circRNAs have been reported to be involved in the biological functions of glioma; however, little is known about the function or molecular mechanisms of the novel circRNA, circATP5B, in glioma, and this circRNA has the potential to become a new molecular diagnostic biomarker and therapeutic target.

Homeobox (HOX) genes constitute a cluster of transcription factors with vital regulatory roles in embryonic development, cell differentiation, and tumorigenesis $(16,17)$. Recent studies confirmed that HOXB5, a member of the homeobox gene family, is overexpressed and promotes cell proliferation and migration in various cancers, including breast, lung, and gastric carcinoma (18-21). However, whether HOXB5 regulates the proliferation of glioma and its specific mechanism in the proliferation of GSCs remains unclear.

MicroRNAs (miRNAs) are characterized as a group of small non-coding RNA fragments (20-25 nucleotides) that can bind to the 3'UTR of mRNAs and lead to translational repression or silencing (22). Previous studies have shown that multiple miRNAs play vital roles in glioma pathogenesis and have potential as prognostic biomarkers (23-25). MiR-185-5p has been confirmed to be downregulated in glioma and acts as a suppressor gene in several biological processes involved in glioma pathogenesis $(26,27)$. Our study speculated that circATP5B might regulate HOXB5 expression through miR-185-5p sponging.

RNA-binding proteins (RBPs) are regulators that participate in RNA synthesis, processing, transport, translation, and degradation (28). Serine/arginine-rich splicing factor 1 (SRSF1) is an RBP that regulates RNA translation, transport, and nonsense-mediated RNA decay (29). SRSF1 has also been identified as a potential oncogene that is overexpressed in several cancers including glioma (29-31). It has been reported that circRNA acts as a sponge for the RBP in glioblastoma and whether SRSF1 can regulate circATP5B expression remains unclear.

Interleukin-6 (IL-6), a pleiotropic proinflammatory cytokine, has been confirmed to play crucial roles in a wide range of biological activities, including immune regulation, inflammation, and oncogenesis through the activation of JAK2/STAT3 signaling (32). In the present study, we firstly found that circATP5B and HOXB5 were overexpressed in glioma, especially in GSCs, and that circATP5B can upregulate HOXB5 expression via miR-185-5p sponging. Furthermore, gene set enrichment analysis (GSEA) showed that higher HOXB5 expression was associated with enrichment of IL6-mediated JAK2/STAT3 signaling according to the TCGA and CGGA datasets. Besides, HOXB5 can transcriptionally regulate the expression of IL-6, and SRSF1 can upregulate circATP5B expression. Therefore, our study found a novel feedback 
loop involving the SRSF1/circATP5B/miR-185-5p/HOXB5 axis that regulates the proliferation of GSCs and may provide a novel target for glioma therapy.

\section{Materials And Methods}

\section{Patient samples and ethical approval}

Seventy clinical samples from glioma patients were collected from January 2007 to January 2012 at the First Affiliated Hospital of China Medical University. There were 20 samples of grade II, 25 samples of grade III, and 25 samples of grade IV glioma. Besides, ten acute brain injury samples from patients were collected during the same period as the control group. Clinical information for these samples is outlined in Supplementary Table 1. This study had obtained the approval of the ethics committee of the First Affiliated Hospital of China Medical University, and every patient wrote informed consent.

\section{Cell culture and GSC isolation}

Six patient-derived primary glioma stem cells from WHO grade II to IV (grade II: GSC201 and GSC203; grade III: GSC302 and GSC305; grade IV: GSC403 and GSC406) were isolated and neurosphere cultures were performed as previously described(33). The detailed clinicopathological information is presented in Supplementary Table 2. In brief, freshly resected glioma samples were dissociated into single cells and cultivated in serum-free DMEM/F12 with $2 \%$ B27, $20 \mathrm{ng} / \mathrm{mL}$ rh-bFGF, and rh-EGF (Gibco, Gaithersburg, MD, USA). The stem cell markers of GSCs were detected by immunofluorescence staining of CD133 (Abcam Technology, Cambridge, UK) and nestin antibodies (Abcam), and the multi-lineage differentiation capacity of GSCs was detected by immunofluorescence staining of GFAP and BIII tubulin (Abcam).

\section{Lentiviral vector construction and transfection}

The lentivirus transfection and efficacy measurements were performed as previously described(33). The lentivirus-based vectors for circATP5B overexpression, HOXB5 overexpression, SRSF1 overexpression, RNAi-mediated knockdown of circATP5B, HOXB5 and SRSF1, and their negative controls were all constructed by Gene-Chem (Shanghai, China). The miR-185-5p mimic, inhibitor, and negative controls were obtained from Thermo Fisher Scientific (Assay ID: MH12486 and MC12486; Thermo Fisher Scientific, Waltham, MA, USA). The sequences of all siRNAs are listed in Supplementary Table 3. The transfection efficacy was detected by qRT-PCR and western blotting.

\section{RNA extraction, nuclear-cytoplasmic fraction, RNase $R$ treatment and quantitative real-time PCR (qRT-PCR)}

qRT-PCR was performed as previously described(33). The total RNA of glioma tissues and GSCs were extracted via the Mini-BEST Universal RNA Extraction kit (TaKaRa, Kyoto, Japan) according to the manufacturer's instructions. For circRNA and relative mRNA, the RNA was reverse transcribed into cDNA through a Prime Script RT Master Mix reagent kit (TaKaRa). For miRNA, cDNA was synthesized by the Prime Script ${ }^{\text {TM }}$ RT reagent kit (TaKaRa, Shiga, Japan). Subsequently, the qRT-PCR assays were detected by 
the SYBR Green Master Mix (TaKaRa) with PCR LightCycler480 (Roche Diagnostics, Basel, Switzerland). The miR-185-5p expression was detected via the TaqMan Universal Master Mix II (Applied Biosystems, Foster

City, CA, USA). Primers used in this study are listed in Supplementary Table 4. In addition, RNase R (Epicentre Technologies, Madison, USA) was used to confirm the existence of circATP5B and eliminate the effect of linear ATP5B RNA. GAPDH, U6 or $\beta$-actin were used as internal controls for circRNA, miRNA or mRNA. Moreover, Nuclear and cytoplasmic RNA fraction was isolated with PARIS ${ }^{\text {TM }}$ Kit (Invitrogen, USA) according to the manufacturer's instruction.

\section{Western blotting}

Western blotting was performed as previously described(33). In brief, a total cell protein extraction kit (KeyGen Biotechnology, Nanjing, China) was used to isolate the total proteins of glioma tissues or GSCs. Then, protein lysates were prepared, and the total protein for each sample was transferred onto the polyvinylidene difluoride (PVDF) membranes after SDS-polyacrylamide gel electrophoresis (SDS-PAGE), and blocked 2 hours at room temperature with $2 \%$ bovine serum albumin (KeyGen Biotechnology). Subsequently, all these membranes were incubated overnight at $4{ }^{\circ} \mathrm{C}$ with the primary antibodies as below: HOXB5 (1:1000; Abcam), IL6 (1:1000; Abcam), p-JAK2 (1:1000; Abcam), JAK2 (1:1000; Abcam), pSTAT3 (1:1000; Abcam), STAT3 (1:1000; Abcam), SRSF1 (1:1000; Abcam) and $\beta$-actin (1:2000; ProteinTech, Chicago, IL, USA). Following 2 hours' secondary antibodies (ProteinTech) incubation, all the bands were detected by a chemiluminescence ECL kit (Beyotime Biotechnology, Beijing, China) and quantified by Image $\mathrm{J}$ software (National Institutes of Health, Bethesda, MD, USA). The relative expression was calculated based on the internal control $\beta$-actin.

\section{Immunohistochemistry (IHC)}

IHC was performed as previously described(33). Firstly, the tumor tissues were embedded in paraffin, sliced into $4 \mathrm{~mm}$ sections, and labeled with primary antibodies as below: HOXB5 (1:100; Abcam), SRSF1 (1:100; Abcam), IL6 (1:100; Abcam) and Ki67 (1:100; Abcam). The slices were then treated with an immunohistochemical labeling kit (MaxVision Biotechnology, Fuzhou, China) and imaged under a light microscope (Olympus, Tokyo, Japan). Finally, the staining intensity and the expression levels were evaluated according to the German immunohistochemical score(34).

\section{Immunofluorescence}

Immunofluorescence staining was performed as previously described(34). Firstly, the GSCs were fixed with $4 \%$ paraformaldehyde (solarbio, Beijing, China) for 10 minutes, permeabilized with $0.5 \%$ Triton X-100 (solarbio) for 20 minutes, blocked with $5 \%$ BSA (solarbio) for 1 hour, and probed with primary antibodies as below: CD133, nestin, GFAP, Blll-tubulin (1:100; Abcam) at $4{ }^{\circ} \mathrm{C}$ overnight. Then, all the samples were treated with fluorescein isothiocyanate or rhodamine-conjugated secondary antibodies. Subsequently, the GSCs were counterstained with DAPI (Sigma, Shanghai, China) for 5 minutes. Finally, the staining was visualized by a laser scanning confocal microscope (Olympus). 


\section{Enzyme-linked immunosorbent (ELISA)}

ELISA was performed as previously described(34). The Abcam's IL-6 Human in vitro ELISA (EnzymeLinked Immunosorbent Assay) kit (ab46027) was used to detect the concentrations of IL6 in the media supernatant of GSCs. All ELISA readings were normalized to the protein concentration in the control groups.

\section{Luciferase reporter assay}

Luciferase reporter assays were performed as described previously(33). Firstly, the luciferase reporter plasmids (circATP5B-wt and circATP5B-mt, HOXB5-3'-UTR-wt and HOXB5-3'-UTR-mt, IL6-wt and IL6-mt, and SRSF1-wt and SRSF1-mt) were constructed by Gene-Chem (Shanghai, China). The GSCs were then seeded into 96 -well plates at a density of $5 \times 10^{3}$ cells/well, transfected with luciferase reporter plasmids and performed other relative treatment for 48 hours. Finally, the relative luciferase activities were detected via a Dual-Luciferase Reporter Assay System (Promega, USA). Relative luciferase activity was calculated as the ratio of firefly luciferase activity to Renilla luciferase activity. All experiments were independently repeated in triplicate.

\section{RNA immunoprecipitation (RIP) assay}

The Imprint RNA Immunoprecipitation Kit (Sigma, USA) was used for RIP assay according to the manufacturer's instructions. All GSCs under different conditions were lysed in RIP buffer including magnetic beads conjugated with negative control IgG, anti-AG02, or anti-SRSF1 antibodies (Millipore, UK). After incubated with Proteinase K buffer (Omega, Shanghai, China), the immunoprecipitated RNAs were obtained. Finally, the qRT-PCR was performed to detect the precipitants.

\section{Chromatin immunoprecipitation (ChIP) assay}

ChIP assays were performed via the ChIP Assay Kit (Beyotime Biotechnology, China) according to the manufacturer's instructions. The chromatin complexes were immunoprecipitated via anti-HOXB5 antibody or normal rabbit lgG, and the purified DNA samples were detected by qRT-PCR. The primers are listed in Supplementary Table 4.

\section{RNA pull-down assay}

The Pierce Magnetic RNA Protein pull-down Kit (Thermo Fisher Scientific) was performed to detect the interaction between circATP5B and SRSF1 according to the manufacturer's instructions. Firstly, purified RNA was labeled by the biotinylated RNA probes, and then the positive control (input), negative control (antisense RNA), and biotinylated RNA were mixed and co-incubated with the proteins of GSCs at room temperature. The RNA-protein complex was added with magnetic beads to prepare a probe-magnetic bead complex. Finally, the complexes were detected by western blotting after being washed and boiled, and $\beta$-actin was used for the control.

\section{Cell viability assay}


Cell viability assays were performed as described previously(33). The GSCs were seeded into 96-well plates at a density of $1 \times 10^{3}$ cells/well and incubated for $0,24,48,72,96$, and 120 hours. The cell viability was then detected via the CellTiter $96 \AA$ Aqueous Non-Radioactive Cell Proliferation Assay Kit (Promega, Madison, WI, USA) according to the manufacturer's instructions.

\section{EDU assay}

EDU assays were performed as described previously(33). According to the manufacturer's instructions, the EDU assay was performed to detect the proliferation of GSCs by the EDU assay kit (Beyotime, Biotechnology, China). Firstly, the GSCs were seeded into 24 -well plates at $1 \times 10^{5}$ cells/well for $24 \mathrm{~h}$, then $10 \mu \mathrm{M}$ EDU reagent was added into the medium and incubated for 2 hours. After being fixed with $4 \%$ paraformaldehyde (solarbio) and permeabilized with $0.5 \%$ Triton X-100 (solarbio), the GSCs were counterstained. Finally, the percentage of EDU positive cells was calculated via a laser scanning confocal microscope (Olympus).

\section{Neurosphere formation assay and in vitro limiting dilution assay}

The neurosphere formation assay was performed as previously described(33). Firstly, the GSCs were seeded into 24-well plates at a density of 200 cells/well and cultured in fresh medium for 7 days. Then, the relative neurosphere size and number of neurospheres were counted via a light microscope (Olympus). For in vitro limiting dilution assay, GSCs were seeded into 96-well plates at a gradient of 1, 10, $20,30,40$ or 50 cells/well, and each gradient replicated 10 times. The number of neurospheres in each well was observed after 7 days incubation, and the neurosphere formation efficiency was calculated via the Extreme Limiting Dilution Analysis (http://bioinf.wehi.edu.au/software/elda)(35).

\section{Xenograft experiments}

Xenograft experiments were performed according to the Animal Care Committee of China Medical University as described previously (33). Firstly, the Six-week-old female BALB/c nude mice (Beijing Vital River Laboratory Animal Technology, Beijing, China) were divided into eight groups: the control, circATP5B-KD1, miR-185-5p-mimic, HOXB5-OE, SRSF1-OE, circATP5B-KD1 + HOXB5-OE, miR-185-5pmimic + HOXB5-OE, SRSF1-OE + circATP5B-KD1 group. Each group with five mice were bred in the Laboratory Animal Center of China Medical University under specific pathogen-free conditions. The GSCs treated with different conditions were orthotopically injected into the mouse brain at $2 \mathrm{~mm}$ lateral and $2 \mathrm{~mm}$ anterior to the bregma by a stereotaxic apparatus $\left(5 \times 10^{4}\right.$ cells per mouse). Each group was observed daily for distress or death signs, the mice were sacrificed and tumors were isolated, the tumor volume was calculated according to the formula: $V=\left(D \times d^{2}\right) / 2$, (D represented the longest diameter and $\mathrm{d}$ represented the shortest diameter). The overall survival time of survived mice was performed KaplanMeier survival analysis.

\section{Bioinformatics analysis}


Gene set enrichment analysis (GSEA, http://www.broadinstitute.org/gsea/index.jsp) was used to analyze the enrichment of signaling pathways between the high and low HOXB5 expression. Four online databases, Starbase (http://starbase.sysu.edu.cn), TargetScan (www. targetscan.org), microRNA (http://www.microrna. org/microrna/home.do), and miRDB (http://mirdb.org) were used to predict possible miRNAs targeting HOXB5. Starbase and circBase (http://www.circbase.org/) databases were used to predict the potential circRNAs as sponges of miRNA and RBPs.

\section{Statistical analysis}

All experiments were repeated at least three times, the results were expressed as the mean \pm SD and the statistical analysis was performed by SPSS 22.0 software (SPSS, Chicago, IL, USA). The comparisons of two independent groups were detected by the chi-square test and two-tailed Student's t-test. The statistical significance among three or more groups was evaluated by One-way analysis of variance. Pearson's correlation analysis was used to detect the correlation between two groups. The Kaplan-Meier analysis and log-rank test were performed to analyze the survival rates of each group. Two-tailed $P$ values $<0.05$ were considered significant.

\section{Results}

CircATP5B upregulation in glioma correlates with poor patient survival.

We first performed qRT-PCR on both normal brain tissue and glioma specimens, and the relative expression of circATP5B in glioma tissue was higher than in normal brain tissue, and was especially increased in higher WHO grade tissues (Fig. 1a). We found that circATP5B, also named hsa_circ_0027068 according to the annotation of circBase (http://www.circbase.org/), was spliced from exons 8 and 9 of the ATP5B gene (chr12: 57031958-57033091) and formed a sense-overlapping circular transcript of 451 nt (Fig. 1b). Sanger sequencing certified the head-to-tail splicing of circATP5B (Fig. 1c). We then determined whether the head-to-tail splicing of circATP5B results from trans-splicing or genomic rearrangement. To certify the stability of circATP5B, both GSC406 and GSC201 were treated with RNase $R$, which is a processive $3^{\prime}$ to $5^{\prime}$ exoribonuclease. It was found that circATP5B resisted digestion by RNase $\mathrm{R}$, but the linear form of ATP5B was readily digested (Fig. 1f). Moreover, the results of nuclear-cytoplasm separation illustrated that circATP5B was predominantly localized in the cytoplasm (Fig. 1g) and indicated that it might be an appropriate diagnostic or prognostic marker. In addition, Kaplan-Meier survival analyses showed that the median survival times of lower-grade glioma patients, glioblastoma multiforme (GBM) patients, or total glioma patients with higher expression of circATP5B were all shorter than those in patients with lower circATP5B expression (Fig. 1e).

We cultured six patient-derived primary GSCs and hematoxylin and eosin were used to stain patientderived glioma tissues (Fig. S1a). Immunofluorescence staining confirmed the enrichment of stem cell markers, CD133 and nestin (Fig. S1b). We also confirmed the differentiation capacity of GSCs with differentiation markers, GFAP and BIII tubulin (Fig. S1C). qRT-PCR showed that the expression of circATP5B was highest in WHO grade IV GSCs (GSC403 and GSC406), followed by WHO grade III GSCs 
(GSC302 and GSC305) and was lowest in WHO grade II GSCs (GSC201 and GSC203) (Figure. 1d). We found that the expression level of circATP5B in GSC406 was the highest and was the lowest in GSC201. Taken together, these results confirmed that circATP5B is overexpressed in glioma and correlates with poor patient survival.

CircATP5B regulates the proliferation of GSCs.

To detect the functions of circATP5B in GSCs, we selected GSC406 and GSC201 for circATP5B silencing or overexpression. qRT-PCR was performed to detect the transfection efficiency (Fig. S2a, b). Then, we evaluated the effects of circATP5B on the proliferation of GSCs via MTS and EDU assays. All of the results showed that the cell viability and EDU-positive rates were decreased in circATP5B-silenced GSC406, while the opposite results were acquired in circATP5B-overexpressed GSC201 (Fig. 1h, i). Furthermore, the relative size of the neurospheres formed by GSC406 was significantly smaller than those of the control group following circATP5B knockdown, while the opposite result was obtained in GSC201 after circATP5B overexpression (Fig. 1j). Limiting dilution assays also showed that the tumor formation incidence decreased in circATP5B-silenced GSC406 but increased in circATP5B-overexpressed GSC201 (Fig. 1k). Together, these results confirmed that circATP5B plays a vital role in promoting the proliferation of GSCs.

HOXB5 is overexpressed in glioma and correlates with poor patient survival.

HOXB5, as a member of the homeobox gene family, is a vital transcription factor, and HOXB5 overexpression is significantly correlated with cancer progression and a poor prognosis $(36,37)$. However, the relationship between HOXB5 and glioma remains largely unknown. We found that the expression levels of HOXB5 in glioma tissues were higher than in normal brain tissues by qRT-PCR, western blotting, and immunohistochemical analysis, and were especially increased in higher glioma WHO grades (Fig. 2a, b, d). Moreover, Kaplan-Meier survival analyses showed that the median survival times of lower-grade glioma patients, GBM patients, or total glioma patients with higher HOXB5 expression were shorter than those for patients with lower HOXB5 expression (Fig. 2c, e, f). Both qRT-PCR and western blotting showed that HOXB5 was most highly expressed in WHO grade IV GSCs (GSC403 and GSC406), followed by WHO grade III GSCs (GSC302 and GSC305) and was lowest in WHO grade II GSCs (GSC201 and GSC203) (Fig. S1d, f). Furthermore, we found that the expression level of HOXB5 was higher in each type of GSC compared with other non-GSC types (Fig. S1e, g). Taken together, these results suggested that HOXB5 is overexpressed in glioma and associated with poor patient survival.

\section{HOXB5 regulates the proliferation of GSCs}

To confirm whether HOXB5 correlated with the proliferation of glioma, we firstly performed qRT-PCR and western blotting to detect the efficiency of HOXB5 knockdown or overexpression (Fig. S2c-f). Then, we performed MTS and EDU assays and the results showed that cell viability and the rates of EDU-positive GSCs were decreased in HOXB5-silenced GSC406 but increased in HOXB5-overexpressed GSC201

(Fig. $2 \mathrm{~g}-\mathrm{i}$ ). Moreover, the relative size of neurospheres formed by GSC406 was significantly smaller than 
those of the control group following HOXB5 knockdown, while the opposite result was obtained in HOXB5-overexpressed GSC201 (Fig. 2k). In addition, limiting dilution assays showed that the incidence of tumor formation decreased in HOXB5-silenced GSC406, but increased in HOXB5-overexpressed GSC201 (Fig. 2j, I). In summary, our findings confirmed that HOXB5 is overexpressed in glioma and actively regulates the proliferation of GSCs.

\section{MiR-185-5p negatively regulates HOXB5 expression.}

We furtherly predicted that miR-185-5p was the only intersection bound to the 3'-UTR of HOXB5 according to microRNA, miRDB, TargetScan, and Starbase databases (Fig. 3a). We performed qRT-PCR and western blotting to confirm whether miR-185-5p regulated HOXB5 expression, and the results showed that HOXB5 expression levels were downregulated in miR-185-5p mimic-treated GSC406 but upregulated in miR-1855p inhibitor-treated GSC201 (Fig. 3b-e). Pearson's correlation analyses also confirmed a negative correlation between the expression levels of miR-185-5p and HOXB5 in each WHO grade glioma and in all glioma samples (Fig. 3h). Furthermore, we constructed luciferase reporter plasmids with wild-type and mutant forms of the HOXB5 3'-UTR (Fig. 3f), and luciferase reporter assays showed that the luciferase activity of HOXB5-wt vector was significantly decreased in miR-185-5p mimic-treated GSC406, while obviously increased in miR-185-5p inhibitor-treated GSC201. However, the luciferase activity of the HOXB5-mt vector showed no significant changes (Fig. 3g). Taken together, these results suggested that miR-185-5p negatively regulates HOXB5 expression through binding to the 3'-UTR of HOXB5.

\section{MiR-185-5p suppresses the proliferation of GSCs via HOXB5 inhibition.}

To confirm the effects of miR-185-5p and HOXB5 in the proliferation of GSCs, we performed rescue experiments. Both MTS and EDU assays showed that the cell viability and rates of EDU-positive GSCs were decreased in miR-185-5p mimic-treated GSC406, while these effects were reversed after HOXB5 overexpression. The opposite results were obtained in miR-185-5p inhibitor-treated GSC201, and the reverse was observed after HOXB5 knockdown (Fig. 3i-k). Furthermore, the relative size of neurospheres formed by GSC406 was significantly smaller than that of the control group after miR-185-5p mimic treatment, but became larger after HOXB5 overexpression. The opposite results were obtained in miR-1855p inhibitor-treated GSC201, and this effect was reversed following HOXB5 knockdown (Fig. 3l). Limiting dilution assays showed that the tumor formation incidence decreased in miR-185-5p mimic-treated GSC406, but increased following HOXB5 overexpression. The opposite results were obtained in miR-185$5 p$ inhibitor-treated GSC201, and the effect was reversed following HOXB5 knockdown (Fig. 3m, n). Together, miR-185-5p negatively regulated HOXB5 expression and suppressed the proliferation of GSCs.

CircATP5B acts as a miRNA sponge of miR-185-5p.

CircRNAs have been confirmed to play crucial roles in several molecular mechanisms, such as miRNAs sponging, protein translation, and RNA-binding protein sponging. Increasing evidence has shown that miRNA sponging is the most common role of circRNAs in the development of tumors, including glioma $(38-41)$. First, we predicted the potential target miRNAs of circATP5B according to Starbase and found 
that miR-185-5p possessed an accurate binding site for circATP5B (Fig. 4a). Second, qRT-PCR showed that the expression of circATP5B was decreased in miR-185-5p mimic-treated GSC406, but increased in miR-185-5p inhibitor-treated GSC201 (Fig. 4b). However, we found that the expression of miR-185-5p increased in circATP5B-silenced GSC406, but decreased in circATP5B-overexpressed GSC201 (Fig. 4c). To confirm the possibility that miR-185-5p binds directly to circATP5B, we constructed luciferase reporter plasmids with wild-type and mutant circATP5B (Fig. 4a). The luciferase activity of circATP5B-wt vector significantly decreased in miR-185-5p mimic-treated GSC406, while obviously increased in miR-185-5p inhibitor-treated GSC201. However, the luciferase activity of the circATP5B-mt vector did not significantly change (Fig. 4d). Moreover, previous studies have shown that miRNAs bind to microRNA response elements (MREs) through RNA-induced silencing complex (RISC), an important component of which is AGO2 protein $(42,43)$. Therefore, we performed an anti-AGO2 RIP assay to determine whether miR-185-5p and circATP5B were co-enriched in the RISC, and the results showed that both circATP5B and miR-185-5p were efficiently pulled down by anti-AGO2 antibody, compared with the IgG group. Furthermore, significant enrichment of circATP5B and miR-185-5p were observed after miR-185-5p mimic treatment, compared with the miR-185-5p negative control group (Fig. 4e). We also found a negative correlation between the expression levels of circATP5B and miR-185-5p in each WHO grade glioma and in all glioma samples via Pearson's correlation analyses (Fig. 4g). In summary, these results demonstrated the direct interaction between circATP5B and miR-185-5p, and indicated that circATP5B may sponge miR-185-5p.

\section{CircATP5B promotes the proliferation of GSCs through miRNA sponging of miR-185-5p}

To confirm the effects of circATP5B and miR-185-5p in the proliferation of GSCs, we performed rescue experiments. MTS and EDU assays showed that cell viability and the rates of EDU-positive GSCs were decreased in circATP5B-silenced GSC406, while this effect was reversed after miR-185-5p inhibitor treatment. However, the opposite results were obtained in circATP5B-overexpressed GSC201, and these upregulations were also reversed after miR-185-5p mimic treatment (Fig. 4f, h). In addition, the relative size of the neurospheres formed by GSC406 was significantly smaller than those of the control group following circATP5B knockdown, but became larger after miR-185-5p inhibitor treatment. While the relative size of the neurospheres formed by GSC201 was obviously larger than that of the control group after circATP5B overexpression, and this reversed following miR-185-5p mimic treatment (Fig. 4j). Limiting dilution assays showed that the tumor formation incidence decreased in circATP5B-silenced GSC406, but increased following miR-185-5p inhibitor treatment. While the opposite results were acquired in circATP5B-overexpressed GSC201, and the increased tumor formation incidence was reversed following miR-185-5p mimic treatment (Fig. 4i, k). Taken together, circATP5B promoted the proliferation of GSCs through sponging miR-185-5p, and there was a negative interaction between circATP5B and miR185-5P.

\section{CircATP5B can upregulate the expression of HOXB5 through miRNA sponging of miR-185-5p}

Page $12 / 49$ 
Since both circATP5B and HOXB5 had specific binding sites for miR-185-5p, to confirm whether circATP5B regulated HOXB5 expression via a miR-185-5p-mediated ceRNA mechanism in GSCs, we firstly detected the expression of HOXB5 via qRT-PCR and western blotting. The results showed that HOXB5 expression was downregulated in circATP5B-silenced GSC406, but upregulated in circATP5Boverexpressed GSC201 (Fig. 5a-C). In addition, we performed rescue experiments by additional treatment with miR-185-5p mimic or miR-185-5p inhibitor. Both the qRT-PCR and western blotting results showed that HOXB5 expression was increased in circATP5B-silenced GSC406 after miR-185-5p inhibitor treatment, while the expression of HOXB5 was decreased in circATP5B-overexpressed GSC201 after miR185-5p mimic treatment (Fig. $5 \mathrm{~d}$-f). Pearson's correlation analyses among clinical glioma specimens showed strong positive correlations between circATP5B and HOXB5 expression in each WHO grade glioma and among the total glioma samples (Fig. 5g). In summary, circATP5B upregulated HOXB5 expression through sponging miR-185-5p.

CircATP5B promotes the proliferation of GSCs by upregulating the expression of HOXB5.

To confirm the effects of circATP5B and HOXB5 in the proliferation of GSCs, we performed rescue experiments. Both MTS and EDU assays showed that cell viability and the rates of EDU-positive GSCs were decreased in circATP5B-silenced GSC406, while these effects were reversed after HOXB5 overexpression. However, cell viability and the rates of EDU-positive GSCs were increased in circATP5Boverexpressed GSC201, and these effects were reversed after HOXB5 knockdown (Fig. 5h-j). Furthermore, the relative size of the neurospheres formed by GSC406 was significantly smaller than that of the control group after circATP5B knockdown, but became larger following HOXB5 overexpression. The opposite results were obtained in circATP5B-overexpressed GSC201, and were reversed after HOXB5 knockdown (Fig. 5k). Limiting dilution assays showed that the incidence of tumor formation decreased in circATP5Bsilenced GSC406, but increased following HOXB5 overexpression. The opposite results were obtained in circATP5B-overexpressed GSC201, but reversed after HOXB5 knockdown (Fig. 5l, m). Taken together, these results suggested that circATP5B actively regulates HOXB5 expression through a miR-185-5pmediated ceRNA mechanism, and promotes the proliferation of GSCs by upregulating HOXB5 expression.

\section{HOXB5 transcriptionally regulates IL6 expression and activates JAK2/STAT3 signaling.}

To confirm the possible downstream mechanism of HOXB5 on glioma, we performed GSEA based on the expression of HOXB5. Both TCGA and CGGA datasets showed that higher HOXB5 expression was associated with enrichment of IL6-mediated JAK2/STAT3 signaling (Fig. 6a). Moreover, Pearson's correlation analyses among clinical glioma specimens revealed significant positive correlations between HOXB5 and IL6 expression in each WHO grade glioma and among the total glioma samples (Fig. 6b). Then, qRT-PCR, western blotting, and ELISA assays showed that IL6 expression was downregulated in HOXB5-silenced GSC406, whereas IL6 expression was upregulated in HOXB5-overexpressed GSC201 (Fig. 6c, d, l, m). Furthermore, since HOXB5 is a transcription factor, we investigated whether HOXB5 transcriptionally regulated the expression of IL6 according to the Jaspar database (Fig. 6e). We performed luciferase reporter assays and found that the luciferase activity of the IL6-wt vector 
significantly decreased in HOXB5-silenced GSC406, while obviously increased in HOXB5-overexpressed GSC201. However, the luciferase activity of the IL6-mt vector showed no significant changes (Fig. 6f). ChIP assays also revealed that the enrichment of IL6 was decreased in GSC406 following HOXB5 knockdown, whereas it was increased in GSC201 after HOXB5 overexpression (Fig. 6g). In addition, we detected the downstream molecules of the JAK2/STAT3 signaling pathway by western blotting and found that the expression levels of p-JAK2 and p-STAT3 were significantly downregulated in HOXB5silenced GSC406, whereas the opposite results were obtained in HOXB5-overexpressed GSC201 (Fig. 6l, m). In summary, HOXB5 could transcriptionally regulate IL6 expression and activate JAK2/STAT3 signaling.

\section{HOXB5 regulates the proliferation of GSCs via IL6/JAK2/STAT3 signaling.}

We furtherly performed rescue experiments to confirm the effects of HOXB5 and IL6 in the proliferation of GSCs. Both MTS and EDU assays showed that cell viability and the rates of EDU-positive GSCs were decreased in HOXB5-silenced GSC406, while these effects were reversed following additional human recombinant IL6 treatment. The opposite results were obtained in HOXB5-overexpressed GSC201, and these effects were also reversed following additional IL6-neutralizing antibody treatment (Fig. 6h, i). In addition, the relative size of neurospheres formed by GSC406 was significantly smaller than that of the control group after HOXB5 knockdown, but became larger following additional human recombinant IL6 treatment. The opposite results were obtained in HOXB5-overexpressed GSC201, and the effects were reversed following additional IL6-neutralizing antibody treatment (Fig. 6j). Limiting dilution assays showed that the incidence of tumor formation decreased in HOXB5-silenced GSC406, but increased after additional human recombinant IL6 treatment. The opposite results were obtained in HOXB5overexpressed GSC201 and reversed following additional IL6-neutralizing antibody treatment (Fig. 6k). Taken together, HOXB5 transcriptionally regulated IL6 expression and promoted the proliferation of GSCs via JAK2/STAT3 signaling.

SRSF1 can bind to and upregulate circATP5B expression.

Splicing factor SRSF1 is a typical splicing factor protein that, in addition to its function in splicing, also plays a crucial role in nonsense-mediated mRNA decay, mRNA export, and translation (44). Splicing is considered the main mechanism by which circRNAs originate, and SRSF1 is upregulated and functions as an oncoprotein in several cancers (45). We found that SRSF1 was the most probable RBP to interact with circATP5B, according to the Starbase database with the highest "ClipExpNum". We firstly selected GSC406 and GSC201 to perform SRSF1 knockdown and overexpression assays, and both qRT-PCR and western blotting were used to detect the efficiency of SRSF1 knockdown or overexpression (Fig. S2g-j). Then, qRT-PCR showed that circATP5B expression was downregulated in SRSF1-silenced GSC406, but upregulated in SRSF1-overexpressed GSC201 (Fig. 7a). Furthermore, we performed a RIP assay to detect whether SRSF1 bound to circATP5B and found that the relative enrichment of circATP5B in the antiSRSF1 group was significantly increased compared with the lgG-treated group. The relative enrichment of circATP5B in the anti-SRSF1 group was obviously decreased after SRSF1 knockdown, but increased after 
SRSF1 overexpression. However, the relative enrichment of circATP5B in the IgG-treated group showed no significant changes (Fig. 7b, c). Moreover, RNA pull-down assays showed that circATP5B-wt pulled down SRSF1 in GSC406 and GSC201, while this effect was not seen with circATP5B-mt (Fig. 7d, e). Together, these results suggested that, as an RBP and splicing factor, SRSF1 promotes the expression of circATP5B.

SRSF1 regulates the proliferation of GSCs by upregulating circATP5B expression.

To confirm the effects of SRSF1 and circATP5B in the proliferation of GSCs, MTS and EDU assays were performed. Both assays showed that cell viability and the rates of EDU-positive GSCs were decreased in SRSF1-silenced GSC406, and these effects were reversed following circATP5B overexpression. The opposite results were obtained in SRSF1-overexpressed GSC201, and the effects were also reversed following circATP5B knockdown (Fig. 7f, h). In addition, the relative size of neurospheres formed by GSC406 was significantly smaller than that of the control group after SRSF1 knockdown, but became larger following circATP5B overexpression. The opposite results were obtained in SRSF1-overexpressed GSC201, and the effects were reversed following circATP5B knockdown (Fig. 7j). Limiting dilution assays showed that the tumor formation incidence decreased in SRSF1-silenced GSC406, but increased after circATP5B overexpression. While the opposite results were obtained in SRSF1-overexpressed GSC201, and these effects were reversed following circATP5B knockdown (Fig. 7i, k). Taken together, SRSF1 promoted the proliferation of GSCs by binding to and upregulating circATP5B expression.

\section{HOXB5 transcriptionally regulates SRSF1 expression in GSCs}

Since HOXB5 is a transcription factor and SRSF1 is an RBP, we determined whether HOXB5 transcriptionally regulates SRSF1 expression in GSCs. We designed two binding sites for HOXB5 in the promoter of SRSF1 according to the Jaspar database (Fig. 7l), then performed luciferase reporter assays. The luciferase activity of SRSF1-wt vector was significantly decreased in HOXB5-silenced GSC406, while obviously increased in HOXB5-overexpressed GSC201. However, the luciferase activity of SRSF1-mt vector showed no obvious changes (Fig. 7m, n). In addition, Pearson's correlation analyses among clinical glioma specimens showed significant positive correlations between HOXB5 and SRSF1 expression in each WHO grade glioma and among the total glioma samples (Fig. 7g). ChIP assays also showed that the enrichment of SRSF1 was decreased in HOXB5-silenced GSC406, while increased in HOXB5-overexpressed GSC201 (Fig. 7o). Finally, qRT-PCR and western blotting showed that SRSF1 expression was downregulated in HOXB5-silenced GSC406, while upregulated in HOXB5-overexpressed GSC201 (Fig. 7p, q, r). Together, these results suggested that HOXB5 transcriptionally regulates SRSF1 expression in GSCs.

The SRSF1/circATP5B/miR-185-5p/HOXB5 feedback loop regulates glioma tumorigenesis in vivo.

We performed orthotopic xenografts to confirm the effects of the SRSF1/circATP5B/miR-185-5p/HOXB5 axis in glioma tumorigenesis in vivo. Compared with the control group, tumor volumes were decreased in 
the circATP5B knockdown group, the miR-185-5p mimic group, and the SRSF1 overexpression combined with circATP5B knockdown group. In contrast, tumor volumes were increased in the HOXB5 overexpression group, the SRSF1 overexpression group, the circATP5B knockdown combined with the HOXB5 overexpression group, and the miR-185-5p mimic combined with the HOXB5 overexpression group (Fig. 8a, b). Kaplan-Meier survival analysis showed similar results with the circATP5B knockdown group, the miR-185-5p mimic group, and the SRSF1 overexpression combined with circATP5B knockdown group showing longer median survival times compared with the normal control group. The opposite results were obtained in the HOXB5 overexpression group, the SRSF1 overexpression group, the circATP5B knockdown combined with HOXB5 overexpression group, and the miR-185-5p mimic combined with the HOXB5 overexpression group (Fig. 8c). Immunohistochemistry was performed to detect the effects of the SRSF1/circATP5B/miR-185-5p/HOXB5 axis on tumor tissues. The results confirmed that the circATP5B knockdown group, the miR-185-5p mimic group, and the SRSF1 overexpression combined with circATP5B knockdown group had lower expression of HOXB5, SRSF1, IL6, and Ki67, whereas higher expression of HOXB5, SRSF1, IL6, and Ki67 was found in the HOXB5 overexpression group, the SRSF1 overexpression group, the circATP5B knockdown combined with HOXB5 overexpression group, and the miR-185-5p mimic combined with the HOXB5 overexpression group (Fig. 8d). To illustrate our findings, the schematic diagram in Fig. 8e shows that the SRSF1/circATP5B/miR-185-5p/HOXB5 feedback loop promotes the tumorigenesis and proliferation of glioma stem cells through the IL6-mediated JAK2/STAT3 signaling pathway. In summary, our results suggested that the SRSF1/circATP5B/miR-185-5p/HOXB5 axis regulates glioma tumorigenesis and proliferation in vivo.

\section{Discussion}

CircRNAs are a type of highly stable and abundant endogenous non-coding RNA formed in the process of RNA splicing. Recently, an increasing number of circRNAs have been confirmed to regulate the development and progression of various human cancers, including breast cancer, hepatocellular carcinoma, and glioma $(11,46-48)$. Several circRNAs are involved in the biological processes of glioma; however, little is known about the role of the novel circRNA, circATP5B, in glioma development and progression. This study found that circATP5B expression was significantly upregulated in clinical glioma specimens and patient-derived primary GSCs. According to Kaplan- Meier survival analyses, the higher expression of circATP5B correlated with a poorer prognosis in glioma patients, especially in higher WHO grade glioma patients. Furthermore, we confirmed the stability of circATP5B and found that it was mainly localized in the cytoplasm. The capacity for active proliferation is regarded as a crucial feature of glioma, which correlates with patients' poor prognosis (11). Functionally, circATP5B promoted the proliferation of GSCs according to MTS, EDU, neurosphere formation, and limiting dilution assays, which implied that circATP5B is a tumor promoter in glioma. CircATP5B has potential as an efficient diagnostic biomarker and therapeutic target for glioma.

CircRNAs have been reported to have diverse molecular mechanisms in the development and progression of various cancers, among which competitive endogenous RNAs are the most frequently reported (12, 
49). CircRNAs contain one or more MREs that act as miRNA sponges to regulate miRNA-targeted genes $(50,51)$. For example, circPTN sponges $\mathrm{miR}-145-5 \mathrm{p} / \mathrm{miR}-330-5 \mathrm{p}$ to promote proliferation and stemness in glioma (11). CircHIPK3 serves as a prognostic marker to promote glioma progression by regulating miR654/IGF2BP3 signaling (41). CircRNA hsa-circ-0014359 promotes glioma progression by regulating miR153/PI3K signaling (2). In our study, we confirmed that both circATP5B and HOXB5 possessed miR-185$5 p$ binding sites, which implied the formation of a circATP5B/miR-185-5p/HOXB5 axis. We first confirmed the direct interaction between circATP5B and miR-185-5p and found that circATP5B negatively regulated miR-185-5p expression according to qRT-PCR, luciferase reporter assays, RIP assays, and Pearson's correlation analyses. Functionally, we also confirmed that circATP5B promoted the proliferation of GSCs and that this effect was reversed by miR-185-5p, which suggested that circATP5B promoted the proliferation of GSCs by acting as a miR-185-5p sponge. Previous studies also demonstrated that miR185-5p was downregulated in glioma, and played an inhibitory role in the development and progression of glioma $(27,52)$.

HOXB5 is a transcription factor that is overexpressed in several cancers and participates in the proliferation, migration, and invasion of cancer cells (18-20). In our study, we certified that HOXB5 was overexpressed in clinical glioma specimens and patient-derived primary GSCs, and the higher expression of HOXB5 correlated with a poorer prognosis in glioma patients, as confirmed by qRT-PCR, western blotting, and Kaplan-Meier survival analyses. Functionally, HOXB5 was also confirmed to promote the proliferation of GSCs. Furthermore, miR-185-5p was shown to bind to the 3'UTR of HOXB5 mRNA, according to luciferase reporter assays, and negatively regulated the expression of HOXB5. We also confirmed that miR-185-5p suppressed the proliferation of GSCs, and that these suppressor effects were reversed by HOXB5, as demonstrated by MTS, EDU, and limiting dilution assays.

We further investigated the relationship between circATP5B and HOXB5 and confirmed that circATP5B actively upregulated HOXB5 expression, as confirmed by qRT-PCR and western blotting. This upregulation could be reversed by treatment with miR-185-5p mimic. Functionally, we also certified that circATP5B promoted the proliferation of GSCs by upregulating HOXB5 expression. Therefore, it is suggested that the circATP5B/miR-185-5p/HOXB5 axis is involved in the proliferation of GSCs.

We further studied the possible downstream effects of HOXB5 and confirmed that higher HOXB5 expression was associated with enrichment of IL6-mediated JAK2/STAT3 signaling. Increasing evidence shows that the activation of IL6-mediated JAK2/STAT3 signaling is frequently associated with glioma, and promotes the cell growth, proliferation, and invasion of glioma cells $(32,53,54)$. In our study, we confirmed that HOXB5 transcriptionally regulated IL6 expression via qRT-PCR and western blotting, as well as ELISA, luciferase reporter, and ChIP assays. We also confirmed significantly positive correlations between HOXB5 and IL6 expression in each WHO grade glioma and among the total glioma samples. In addition, we showed that HOXB5 promoted the expression of downstream molecules of the JAK2/STAT3 signaling pathway by western blotting. Functionally, we confirmed that HOXB5 promoted the proliferation of GSCs via activating IL6-mediated JAK2/STAT3 signaling. 
RBPs are involved in the post-transcriptional regulation of RNAs, as well as gene transcription and translation. They also play vital roles in both physiological and pathological processes (55). RBPs have also been reported to participate in circRNA splicing and expression and promote the production of circRNAs $(56,57)$. SRSF1, a splicing factor and a type of RBP, has been reported to be overexpressed in several cancers including glioma, and participates in diverse biological functions, including translation, nonsense-mediated RNA decay, and RNA transport (29-31). In our study, we confirmed that SRSF1 bound to circATP5B and promoted circATP5B expression in GSCs via qRT-PCR, RIP and RNA pull-down assays. SRSF1 did not affect the expression of the ATP5B linear form. Functionally, we confirmed that SRSF1 promoted the proliferation of GSCs by binding to circATP5B, and synergetic effects were detected between SRSF1 and circATP5B in the proliferation of GSCs through MTS, EDU, and limiting dilution assays.

As a transcriptional factor, our study found two binding sites for HOXB5 in the promoter of SRSF1 and confirmed that HOXB5 transcriptionally regulated SRSF1 expression in GSCs. We also certified that there were significant positive correlations between HOXB5 and SRSF1 expression in clinical glioma specimens. Finally, we conclude that the SRSF1/circATP5B/miR-185-5p/HOXB5 feedback loop is involved in glioma tumorigenesis and proliferation.

\section{Conclusions}

In summary, circATP5B was upregulated in glioma and this correlated with poor patient survival. We confirmed that circATP5B promoted the proliferation of glioma using patient-derived GSCs. Furthermore, HOXB5 transcriptionally regulated IL6 expression, and promoted the proliferation of GSCs via IL6mediated JAK2/STAT3 signaling. Mechanistically, circATP5B upregulated the expression of HOXB5 in GSCs via miR-185-5p sponging. In addition, SRSF1 bound to circATP5B and promoted circATP5B expression, while HOXB5 also transcriptionally regulated and promoted SRSF1 expression in GSCs. Therefore, the SRSF1/circATP5B/miR-185-5p/HOXB5 feedback loop is considered to be involved in glioma proliferation. Our study has identified a novel potential biomarker for glioma diagnosis and prognosis evaluation, and may also offer a new target for glioma treatment.

\section{Abbreviations}

Glioma stem cells: GSCs; Circular RNA: circRNA; Homeobox: HOX; MicroRNAs: miRNAs; RNA-binding proteins: RBPs; interleukin-6: IL6; Gene set enrichment analysis: GSEA; The Cancer Genome Atlas:TCGA ; Chinese Glioma Genome Atlas:CGGA ; Real-Time Quantitative Reverse Transcription PCR: qRT-PCR; Immunohistochemistry: IHC; Enzyme-linked immunosorbent assay: ELISA; RNA immunoprecipitation: RIP; Chromatin immunoprecipitation: ChIP; RNA-induced silencing complex: RISC; competitive endogenous RNAs: ceRNAs; lower-grade glioma: LGG; glioblastoma: GBM.

\section{Declarations}




\section{Ethics approval and consent to participate}

Patients and controls were acquired with informed consent, under the protocol approved by the First Hospital of China Medical University research ethics committee.

\section{Consent for publication}

Consent to publish has been obtained from all authors.

\section{Availability of data and materials}

The datasets obtained and analyzed during the current study were made available from the corresponding authors through request.

\section{Conflict of interest}

The authors declare no potential conflicts of interest.

\section{Funding}

This work was supported by the National Natural Science Foundation of China (Nos. 82072794), the Major Disease Prevention and Control Technology Action Plan of China (2018ZX-07S-006), the Liaoning BaiQianWan Talents Program (No. 2019-B45), the Social Development Program from Shenyang Science and Technology Bureau, China (20-205-4-075), the Project of JiangSu XianSheng Pharmaceutical Co. (JSXSZD-SA2020-06-004).

\section{Authors' contributions}

ZTJ conceived and designed the study; JSZ and YJ performed the experiments; HYZ, JPZ and LC performed bioinformatics analysis; $\mathrm{HL}$, JKX and GQZ collected and analyzed the data. JSZ and YJ interpreted the results and wrote the manuscript. JSZ and YJ contributed equally to this work. All authors read and approved the final version of the manuscript.

\section{Acknowledgments}

We would like to acknowledge our lab colleagues for their support in the development of this article.

\section{References}

1. Zhou Q, Liu J, Quan J, Liu W, Tan H, Li W. MicroRNAs as potential biomarkers for the diagnosis of glioma: A systematic review and meta-analysis. Cancer Sci. 2018;109(9):2651-9.

2. Shi F, Shi Z, Zhao Y, Tian J. CircRNA hsa-circ-0014359 promotes glioma progression by regulating miR-153/PI3K signaling. Biochem Biophys Res Commun. 2019;510(4):614-20.

3. Hombach-Klonisch S, Mehrpour M, Shojaei S, Harlos C, Pitz M, Hamai A, et al. Glioblastoma and chemoresistance to alkylating agents: Involvement of apoptosis, autophagy, and unfolded protein response. Pharmacol Ther. 2018;184:13-41.

4. Zhan X, Guo S, Li Y, Ran H, Huang H, Mi L, et al. Glioma stem-like cells evade interferon suppression through MBD3/NuRD complex-mediated STAT1 downregulation. The Journal of experimental medicine. 2020;217(5). 
5. Chen S, Huang V, Xu X, Livingstone J, Soares F, Jeon J, et al. Widespread and Functional RNA Circularization in Localized Prostate Cancer. Cell. 2019;176(4):831-43.e22.

6. Bhandari V, Hoey C, Liu LY, Lalonde E, Ray J, Livingstone J, et al. Molecular landmarks of tumor hypoxia across cancer types. Nat Genet. 2019;51(2):308-18.

7. Han YN, Xia SQ, Zhang YY, Zheng JH, Li W. Circular RNAs: A novel type of biomarker and genetic tools in cancer. Oncotarget. 2017;8(38):64551-63.

8. Zhang M, Zhao K, Xu X, Yang Y, Yan S, Wei P, et al. A peptide encoded by circular form of LINC-PINT suppresses oncogenic transcriptional elongation in glioblastoma. Nat Commun. 2018;9(1):4475.

9. Yu J, Xu QG, Wang ZG, Yang Y, Zhang L, Ma JZ, et al. Circular RNA cSMARCA5 inhibits growth and metastasis in hepatocellular carcinoma. Journal of hepatology. 2018;68(6):1214-27.

10. Hsiao KY, Lin YC, Gupta SK, Chang N, Yen L, Sun HS, et al. Noncoding Effects of Circular RNA CCDC66 Promote Colon Cancer Growth and Metastasis. Cancer Res. 2017;77(9):2339-50.

11. Chen J, Chen T, Zhu Y, Li Y, Zhang Y, Wang Y, et al. circPTN sponges miR-145-5p/miR-330-5p to promote proliferation and stemness in glioma. J Exp Clin Cancer Res. 2019;38(1):398.

12. Qu Y, Zhu J, Liu J, Qi L. Circular RNA circ_0079593 indicates a poor prognosis and facilitates cell growth and invasion by sponging miR-182 and miR-433 in glioma. J Cell Biochem. 2019;120(10):18005-13.

13. Kristensen LS, Hansen TB, Ven $\varnothing$ MT, Kjems J. Circular RNAs in cancer: opportunities and challenges in the field. Oncogene. 2018;37(5):555-65.

14. Rong D, Sun H, Li Z, Liu S, Dong C, Fu K, et al. An emerging function of circRNA-miRNAs-mRNA axis in human diseases. Oncotarget. 2017;8(42):73271-81.

15. Li Y, Zheng Q, Bao C, Li S, Guo W, Zhao J, et al. Circular RNA is enriched and stable in exosomes: a promising biomarker for cancer diagnosis. Cell Res. 2015;25(8):981-4.

16. Mallo $\mathrm{M}$, Alonso $\mathrm{CR}$. The regulation of Hox gene expression during animal development. Development. 2013;140(19):3951-63.

17. Armat M, Ramezani F, Molavi O, Sabzichi M, Samadi N. Six family of homeobox genes and related mechanisms in tumorigenesis protocols. Tumori. 2016;2016(3):236-43.

18. Lee JY, Hur H, Yun HJ, Kim Y, Yang S, Kim SI, et al. HOXB5 Promotes the Proliferation and Invasion of Breast Cancer Cells. Int J Biol Sci. 2015;11(6):701-11.

19. Zhang B, Li N, Zhang H. Knockdown of Homeobox B5 (HOXB5) Inhibits Cell Proliferation, Migration, and Invasion in Non-Small Cell Lung Cancer Cells Through Inactivation of the Wnt/ $\beta$-Catenin Pathway. Oncol Res. 2018;26(1):37-44.

20. Hong CS, Jeong O, Piao Z, Guo C, Jung MR, Choi C, et al. HOXB5 induces invasion and migration through direct transcriptional up-regulation of $\beta$-catenin in human gastric carcinoma. Biochem $\mathrm{J}$. 2015;472(3):393-403.

21. Luo J, Cai Q, Wang W, Huang $\mathrm{H}$, Zeng $\mathrm{H}$, He W, et al. A microRNA-7 binding site polymorphism in HOXB5 leads to differential gene expression in bladder cancer. PLoS One. 2012;7(6):e40127. 
22. Agarwal V, Bell GW, Nam JW, Bartel DP. Predicting effective microRNA target sites in mammalian mRNAs. Elife. 2015;4.

23. He X, Fan S. hsa-miR-212 modulates the radiosensitivity of glioma cells by targeting BRCA1. Oncol Rep. 2018;39(3):977-84.

24. Li L, Zhang H. MicroRNA-379 inhibits cell proliferation and invasion in glioma via targeting metadherin and regulating PTEN/AKT pathway. Mol Med Rep. 2018;17(3):4049-56.

25. Wang L, Liu Y, Song J. MicroRNA-103 suppresses glioma cell proliferation and invasion by targeting the brain-derived neurotrophic factor. Mol Med Rep. 2018;17(3):4083-9.

26. Agrawal R, Pandey P, Jha P, Dwivedi V, Sarkar C, Kulshreshtha R. Hypoxic signature of microRNAs in glioblastoma: insights from small RNA deep sequencing. BMC Genomics. 2014;15(1):686.

27. Ni W, Xia Y, Bi Y, Wen F, Hu D, Luo L. FoxD2-AS1 promotes glioma progression by regulating miR-1855P/HMGA2 axis and PI3K/AKT signaling pathway. Aging (Albany NY). 2019;11(5):1427-39.

28. Castello A, Fischer B, Eichelbaum K, Horos R, Beckmann BM, Strein $C$, et al. Insights into RNA biology from an atlas of mammalian mRNA-binding proteins. Cell. 2012;149(6):1393-406.

29. Sheng J, Zhao Q, Zhao J, Zhang W, Sun Y, Qin P, et al. SRSF1 modulates PTPMT1 alternative splicing to regulate lung cancer cell radioresistance. EBioMedicine. 2018;38:113-26.

30. Anczukow O, Akerman M, Clery A, Wu J, Shen C, Shirole NH, et al. SRSF1-Regulated Alternative Splicing in Breast Cancer. Mol Cell. 2015;60(1):105-17.

31. Zhou X, Li X, Yu L, Wang R, Hua D, Shi C, et al. The RNA-binding protein SRSF1 is a key cell cycle regulator via stabilizing NEAT1 in glioma. Int J Biochem Cell Biol. 2019;113:75-86.

32. Cheng P, Wang J, Waghmare I, Sartini S, Coviello V, Zhang Z, et al. FOXD1-ALDH1A3 Signaling Is a Determinant for the Self-Renewal and Tumorigenicity of Mesenchymal Glioma Stem Cells. Cancer research. 2016;76(24):7219-30.

33. Jiang Y, Zhou J, Zhao J, Hou D, Zhang H, Li L, et al. MiR-18a-downregulated RORA inhibits the proliferation and tumorigenesis of glioma using the TNF-alpha-mediated NF-kappaB signaling pathway. EBioMedicine. 2020;52:102651.

34. Jiang Y, Zhou J, Luo P, Gao H, Ma Y, Chen YS, et al. Prosaposin promotes the proliferation and tumorigenesis of glioma through toll-like receptor 4 (TLR4)-mediated NF-kappaB signaling pathway. EBioMedicine. 2018;37:78-90.

35. Hu Y, Smyth GK. ELDA: extreme limiting dilution analysis for comparing depleted and enriched populations in stem cell and other assays. Journal of immunological methods. 2009;347(1-2):70-8.

36. Lee K, Chang JW, Oh C, Liu L, Jung S-N, Won H-R, et al. HOXB5 acts as an oncogenic driver in head and neck squamous cell carcinoma via EGFR/Akt/Wnt/ $\beta$-catenin signaling axis. European Journal of Surgical Oncology. 2019.

37. Li ZX, Wu G, Jiang WJ, Li J, Wang YY, Ju XM, et al. HOXB5 promotes malignant progression in pancreatic cancer via the miR-6732 pathway. Cell Cycle. 2020;19(2):233-45. 
38. Kristensen LS, Hansen TB, Veno MT, Kjems J. Circular RNAs in cancer: opportunities and challenges in the field. Oncogene. 2018;37(5):555-65.

39. Zhu Z, Yu Z, Rong Z, Luo Z, Zhang J, Qiu Z, et al. The novel GINS4 axis promotes gastric cancer growth and progression by activating Rac1 and CDC42. Theranostics. 2019;9(26):8294-311.

40. Zheng J, Liu X, Xue Y, Gong W, Ma J, Xi Z, et al. TTBK2 circular RNA promotes glioma malignancy by regulating miR-217/HNF1beta/Derlin-1 pathway. Journal of hematology \& oncology. 2017;10(1):52.

41. Jin P, Huang Y, Zhu P, Zou Y, Shao T, Wang O. CircRNA circHIPK3 serves as a prognostic marker to promote glioma progression by regulating miR-654/IGF2BP3 signaling. Biochem Biophys Res Commun. 2018;503(3):1570-4.

42. Zhu Z, Rong Z, Luo Z, Yu Z, Zhang J, Qiu Z, et al. Circular RNA circNHSL1 promotes gastric cancer progression through the miR-1306-3p/SIX1/vimentin axis. Molecular Cancer. 2019;18(1).

43. Gregory RI, Chendrimada TP, Cooch N, Shiekhattar R. Human RISC couples microRNA biogenesis and posttranscriptional gene silencing. Cell. 2005;123(4):631-40.

44. Das S, Krainer AR. Emerging functions of SRSF1, splicing factor and oncoprotein, in RNA metabolism and cancer. Mol Cancer Res. 2014;12(9):1195-204.

45. Barbagallo D, Caponnetto A, Brex D, Mirabella F, Barbagallo C, Lauretta G, et al. CircSMARCA5 Regulates VEGFA mRNA Splicing and Angiogenesis in Glioblastoma Multiforme Through the Binding of SRSF1. Cancers (Basel). 2019;11(2).

46. Gao D, Qi X, Zhang X, Fang K, Guo Z, Li L. hsa_circRNA_0006528 as a competing endogenous RNA promotes human breast cancer progression by sponging miR-7-5p and activating the MAPK/ERK signaling pathway. Mol Carcinog. 2019;58(4):554-64.

47. Xu L, Feng X, Hao X, Wang P, Zhang Y, Zheng X, et al. CircSETD3 (Hsa_circ_0000567) acts as a sponge for microRNA-421 inhibiting hepatocellular carcinoma growth. J Exp Clin Cancer Res. 2019;38(1):98.

48. Peng H, Qin C, Zhang C, Su J, Xiao Q, Xiao Y, et al. circCPA4 acts as a prognostic factor and regulates the proliferation and metastasis of glioma. J Cell Mol Med. 2019;23(10):6658-65.

49. He Q, Zhao L, Liu Y, Liu X, Zheng J, Yu H, et al. circ-SHKBP1 Regulates the Angiogenesis of U87 Glioma-Exposed Endothelial Cells through miR-544a/FOXP1 and miR-379/FOXP2 Pathways. Molecular therapy Nucleic acids. 2018;10:331-48.

50. Piwecka M, Glažar P, Hernandez-Miranda LR, Memczak S, Wolf SA, Rybak-Wolf A, et al. Loss of a mammalian circular RNA locus causes miRNA deregulation and affects brain function. Science. 2017;357(6357).

51. Zheng Q, Bao C, Guo W, Li S, Chen J, Chen B, et al. Circular RNA profiling reveals an abundant circHIPK3 that regulates cell growth by sponging multiple miRNAs. Nat Commun. 2016;7:11215.

52. Shen F, Chang H, Gao G, Zhang B, Li X, Jin B. Long noncoding RNA FOXD2-AS1 promotes glioma malignancy and tumorigenesis via targeting miR-185-5p/CCND2 axis. J Cell Biochem. 2019;120(6):9324-36. 
53. Hou J, Lv A, Deng Q, Zhang G, Hu X, Cui H. TROP2 promotes the proliferation and metastasis of glioblastoma cells by activating the JAK2/STAT3 signaling pathway. Oncol Rep. 2019;41(2):753-64.

54. Zhong C, Tao B, Chen Y, Guo Z, Yang X, Peng L, et al. B7-H3 Regulates Glioma Growth and Cell Invasion Through a JAK2/STAT3/Slug-Dependent Signaling Pathway. Onco Targets Ther. 2020;13:2215-24.

55. Zang J, Lu D, Xu A. The interaction of circRNAs and RNA binding proteins: An important part of circRNA maintenance and function. Journal of neuroscience research. 2020;98(1):87-97.

56. Conn SJ, Pillman KA, Toubia J, Conn VM, Salmanidis M, Phillips CA, et al. The RNA binding protein quaking regulates formation of circRNAs. Cell. 2015;160(6):1125-34.

57. Lyu D, Huang S. The emerging role and clinical implication of human exonic circular RNA. RNA biology. 2017;14(8):1000-6.

\section{Figures}


a

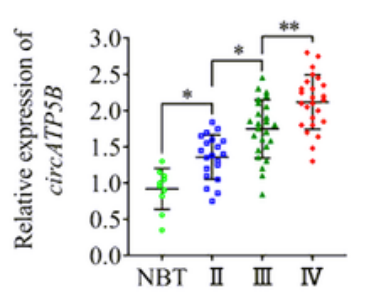

e

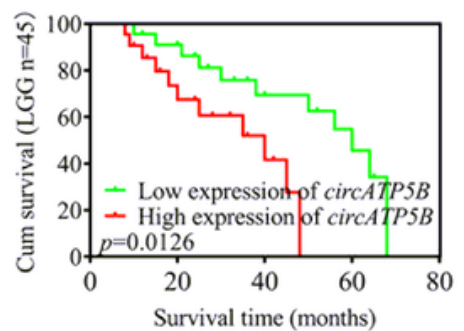

f
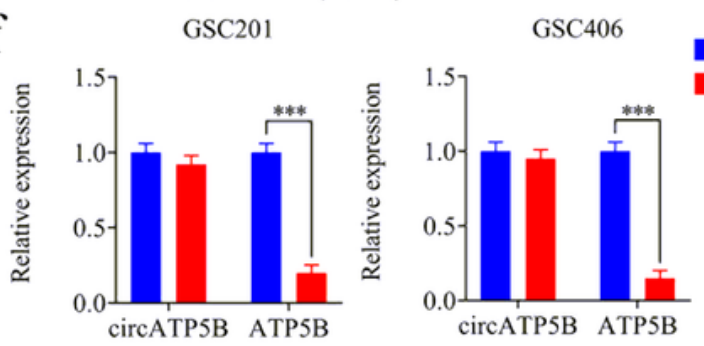

b
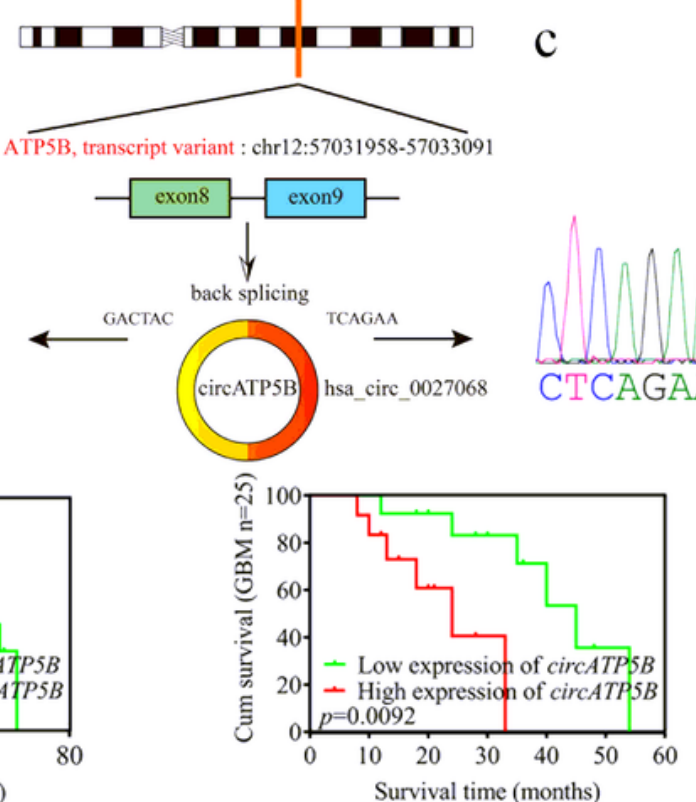

Survival time (months) $\mathrm{c}$

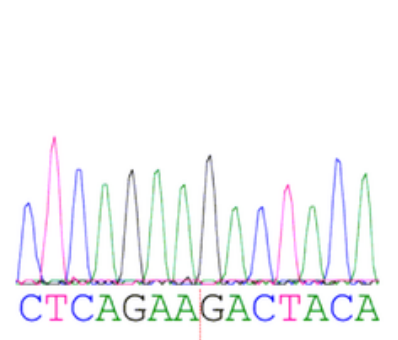

d

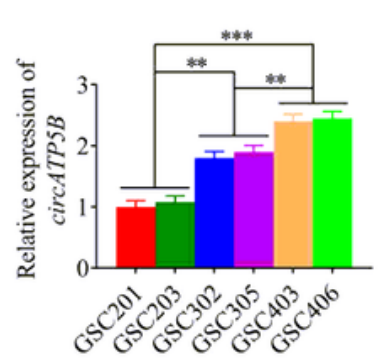

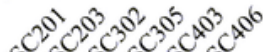

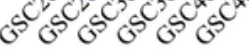

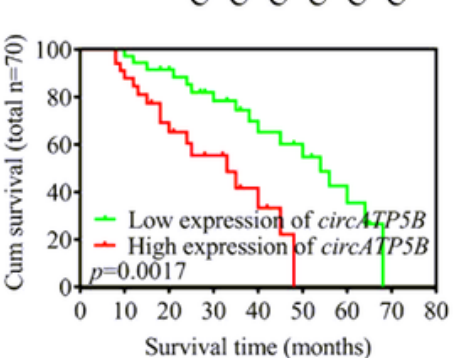

- g GSC201 Survival time (months)

Mock
RNase R
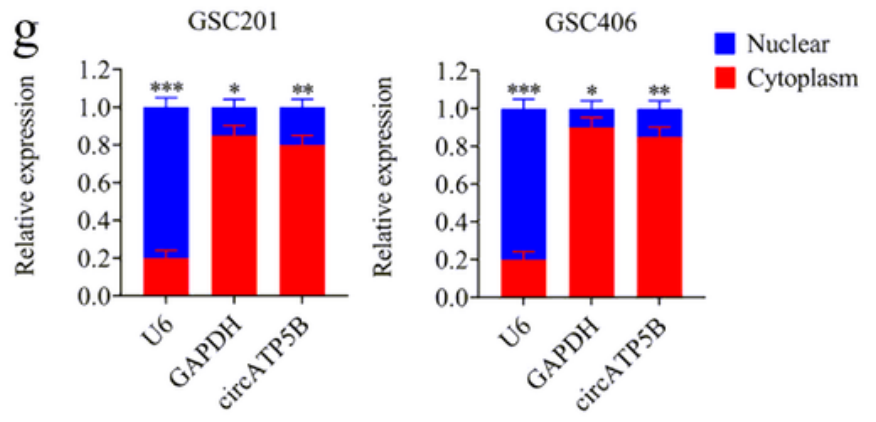

h $\quad$ GSC406
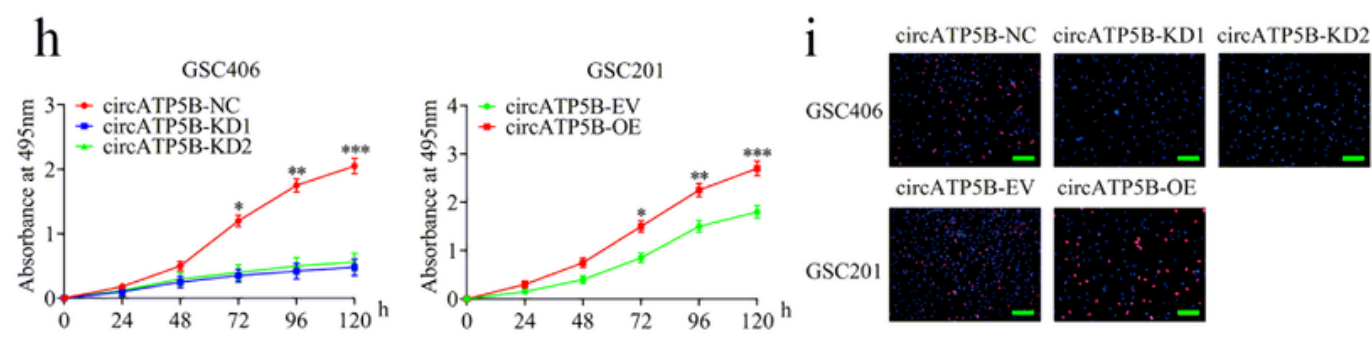

circATP5B-NC

circATP5B-KD1 0 circATP5B-EV circATP5B-KD2

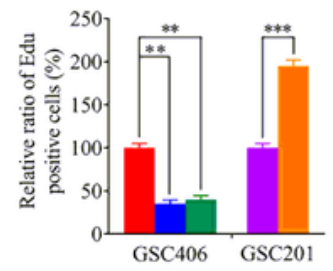

$\mathrm{j}$
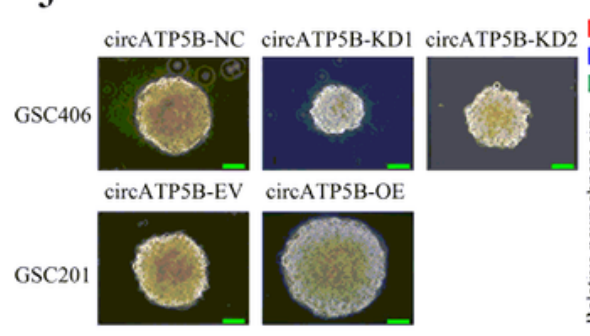

$\mathrm{k}_{\circ}$
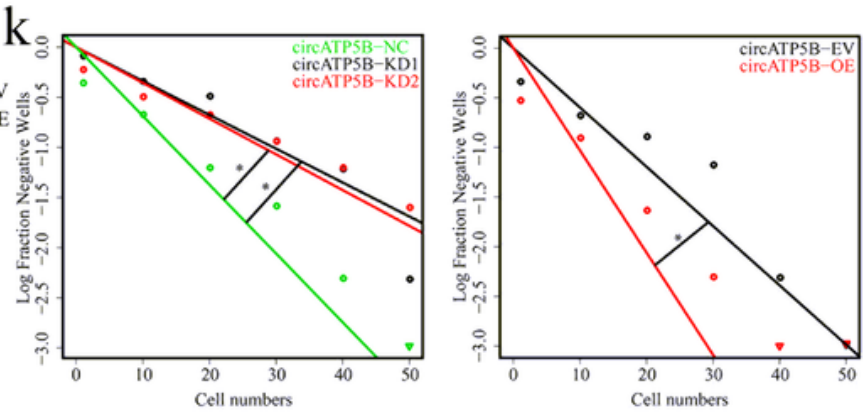

Figure 1

CircATP5B was upregulated in glioma and correlated with poor patient survival, and regulated the proliferation of GSCs. a CircATP5B was expressed at higher levels in different grade glioma tissues, compared with normal brain tissues as detected by qRT-PCR. (NBT, $n=10$; grade II, $n=20$; grade III, $n=$ 25 ; grade IV, $n=25$; one-way analysis of variance). b Schematic illustration of the formation of circATP5B via the circularization of exons in the ATP5B gene. c Sanger sequencing confirmed the head-to-tail 
splicing of circATP5B. d CircATP5B was expressed at higher levels in high grade patient-derived primary GSCs as detected by qRT-PCR. e Kaplan-Meier analysis showed the prognostic significance of the 70 glioma patients, 45 LGG patients, and 25 GBM patients with high versus low circATP5B expression detected by qRT-PCR. $f$ Relative expressions of circATP5B and ATP5B in both GSC406 and GSC201 were detected by qRT-PCR in the presence or absence of RNase R. $g$ circATP5B was mainly located in the cytoplasm by nuclear-cytoplasmic fractionation assay. h MTS assays showed that circATP5B knockdown or overexpression affected the cell viability of GSCs (one-way analysis of variance). $i$ The EDU assays showed that circATP5B knockdown or overexpression affected the proliferation of GSCs (Student's t-test). Scale bar $=50 \mu \mathrm{m}$. j The neurospheres formation assays showed that circATP5B knockdown or overexpression affected the self-renewing capacity of GSCs (Student's t-test). Scale bar = $20 \mu \mathrm{m}$. $\mathrm{k}$ circATP5B knockdown or overexpression affected the tumor formation incidence of GSCs as detected by limiting dilution assays (Student's t-test). EV: empty vector, OE: overexpression, NC: negative control, KD: knockdown. All data were expressed as the mean $\pm \mathrm{SD}$ (three independent experiments). ${ }^{*} \mathrm{p}<0.05 ;{ }^{* \star} \mathrm{p}<0.01 ; * \star * \mathrm{p}<0.001$. 
$\mathrm{a}$

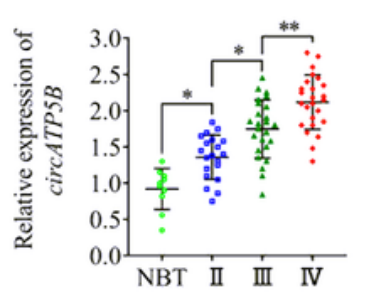

e

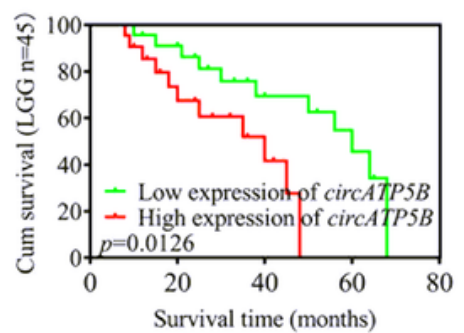

$\mathrm{f}$
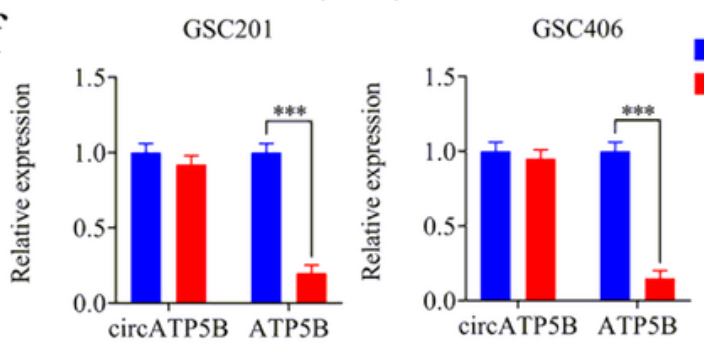

b
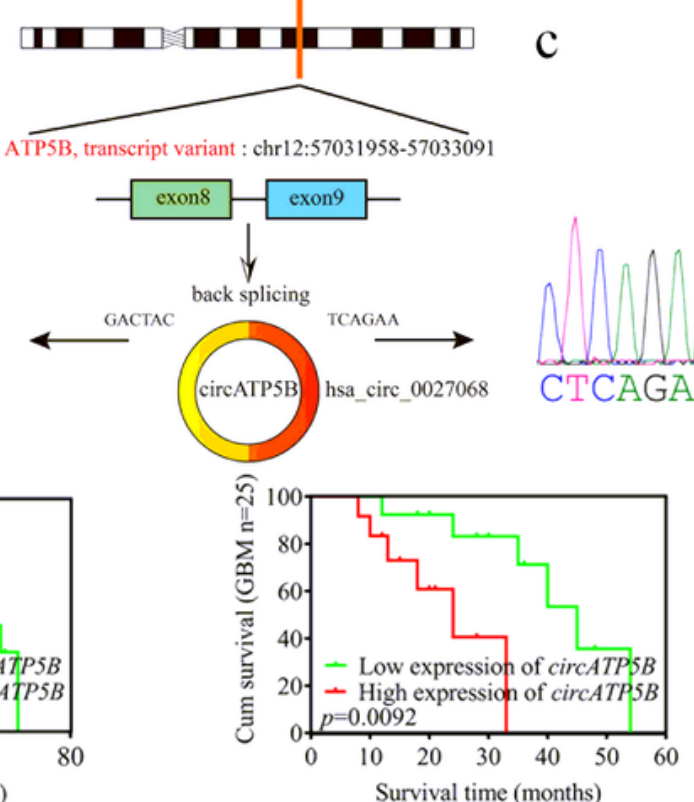

Survival time (months) $\mathrm{c}$

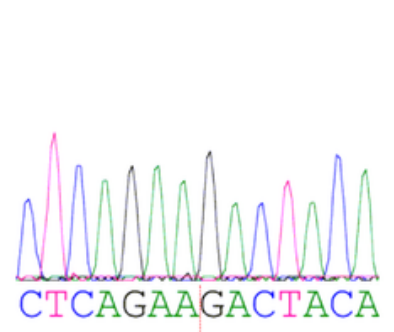

d

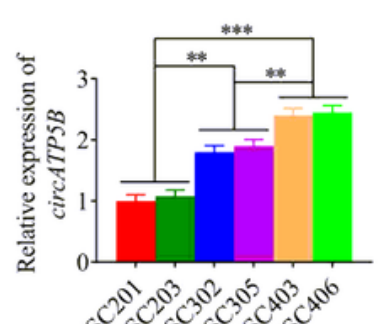

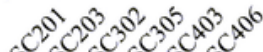

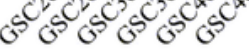

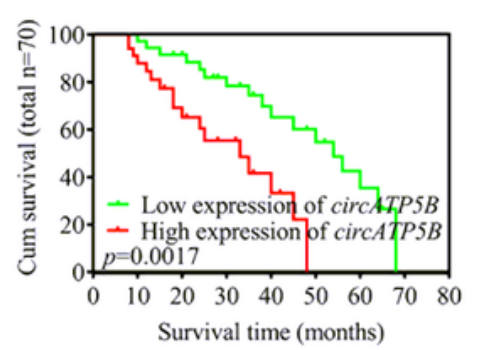

g GSC201

GSC406

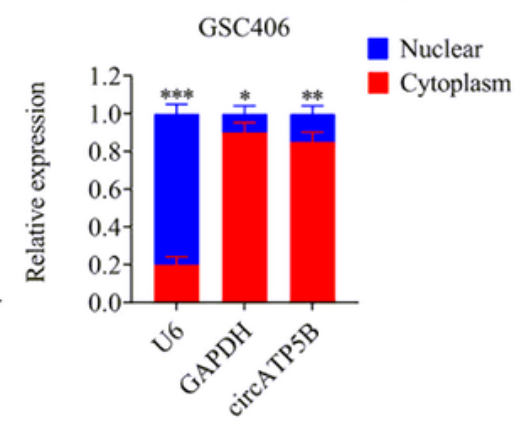

h $\quad$ GSC406
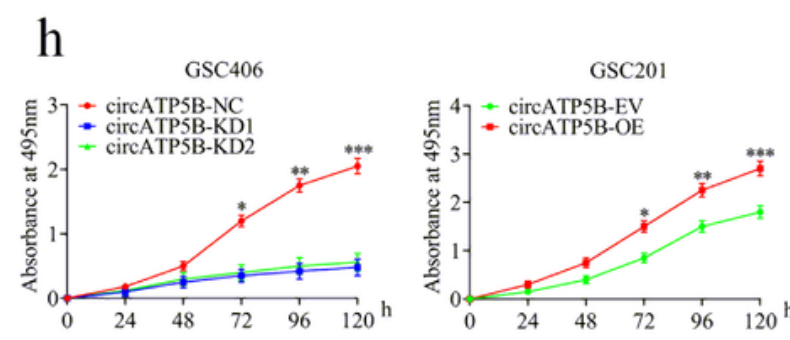

i circATPSB-NC circATPSB-KD1 circATPSB-KD2

circATP5B-NC

circATP5B-KD1 0 circATP5B-EV circATP5B-KD2
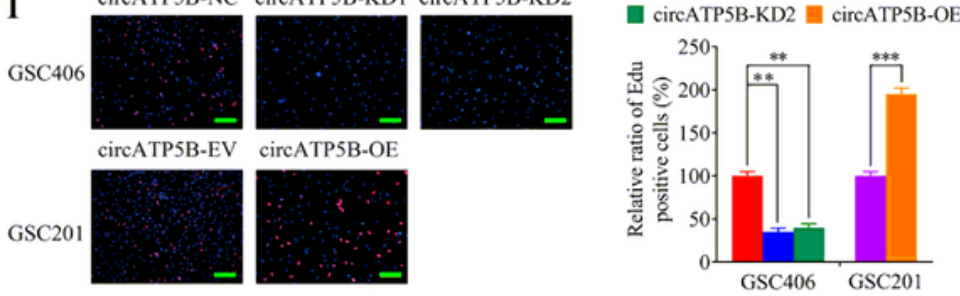

$\mathrm{j}$
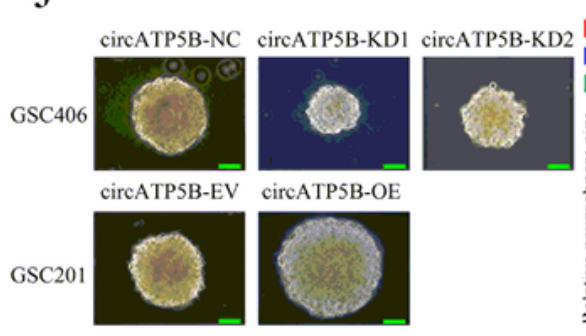

$\mathrm{k}_{\circ}$
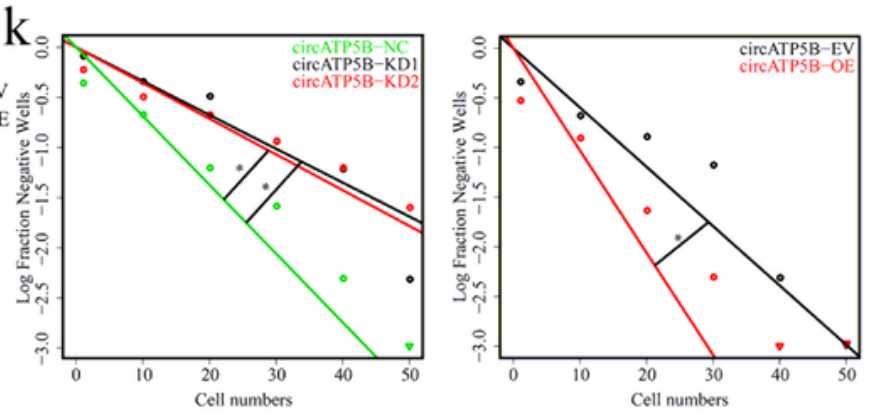

Figure 1

CircATP5B was upregulated in glioma and correlated with poor patient survival, and regulated the proliferation of GSCs. a CircATP5B was expressed at higher levels in different grade glioma tissues, compared with normal brain tissues as detected by qRT-PCR. (NBT, $n=10$; grade II, $n=20$; grade III, $n=$ 25 ; grade IV, $n=25$; one-way analysis of variance). b Schematic illustration of the formation of circATP5B via the circularization of exons in the ATP5B gene. c Sanger sequencing confirmed the head-to-tail 
splicing of circATP5B. d CircATP5B was expressed at higher levels in high grade patient-derived primary GSCs as detected by qRT-PCR. e Kaplan-Meier analysis showed the prognostic significance of the 70 glioma patients, 45 LGG patients, and 25 GBM patients with high versus low circATP5B expression detected by qRT-PCR. $f$ Relative expressions of circATP5B and ATP5B in both GSC406 and GSC201 were detected by qRT-PCR in the presence or absence of RNase R. $g$ circATP5B was mainly located in the cytoplasm by nuclear-cytoplasmic fractionation assay. h MTS assays showed that circATP5B knockdown or overexpression affected the cell viability of GSCs (one-way analysis of variance). $i$ The EDU assays showed that circATP5B knockdown or overexpression affected the proliferation of GSCs (Student's t-test). Scale bar $=50 \mu \mathrm{m}$. j The neurospheres formation assays showed that circATP5B knockdown or overexpression affected the self-renewing capacity of GSCs (Student's t-test). Scale bar = $20 \mu \mathrm{m}$. $\mathrm{k}$ circATP5B knockdown or overexpression affected the tumor formation incidence of GSCs as detected by limiting dilution assays (Student's t-test). EV: empty vector, OE: overexpression, NC: negative control, KD: knockdown. All data were expressed as the mean $\pm \mathrm{SD}$ (three independent experiments). ${ }^{*} \mathrm{p}<0.05 ;{ }^{* \star} \mathrm{p}<0.01 ; * \star * \mathrm{p}<0.001$. 
a
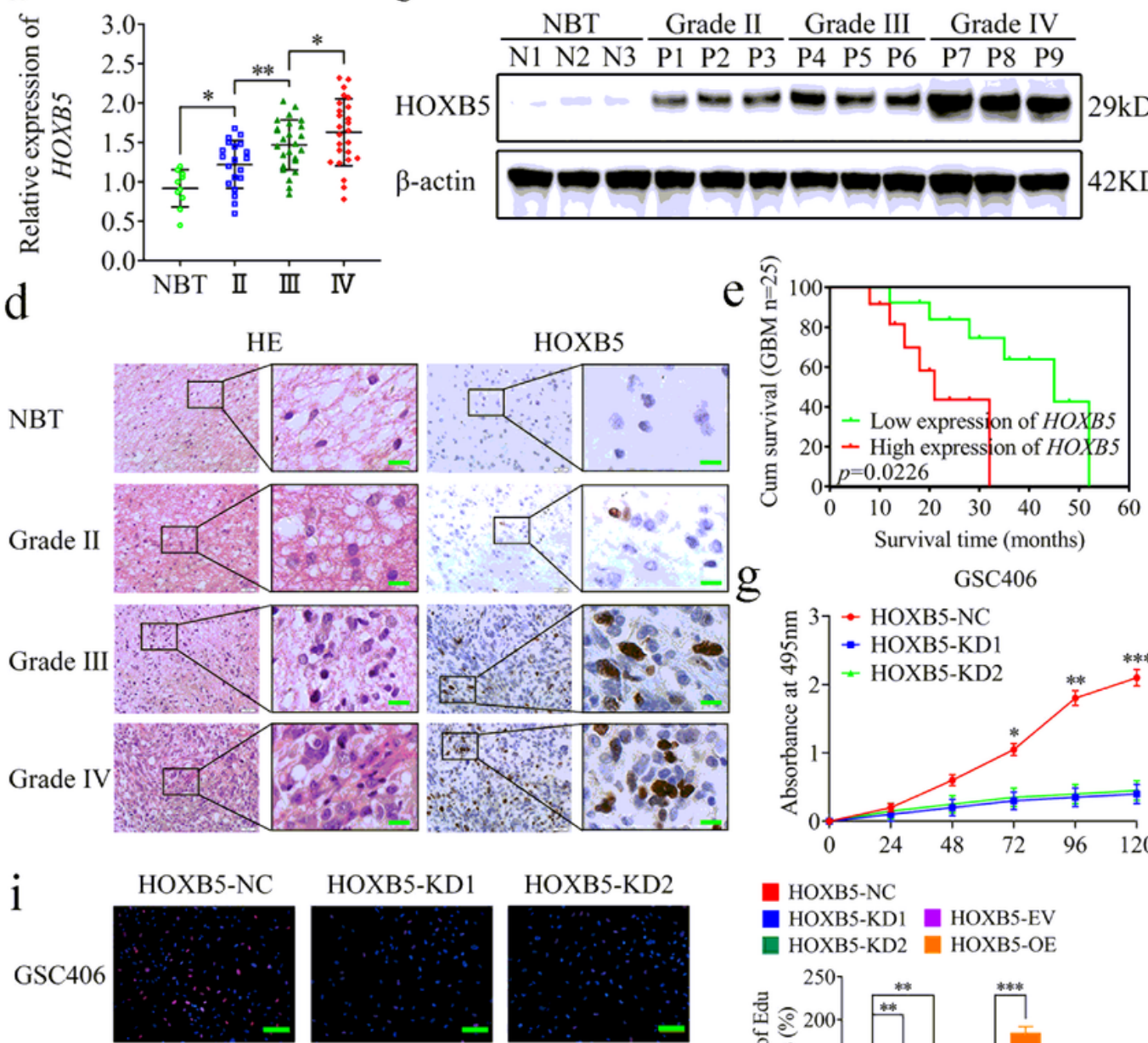

$$
\text { HOXB5-EV }
$$

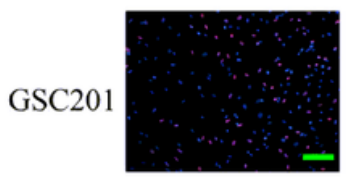

k

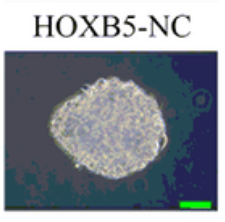

HOXB5-EV

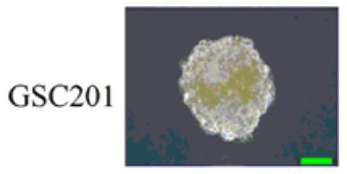

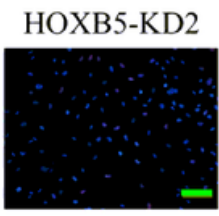

HOXB5-OE

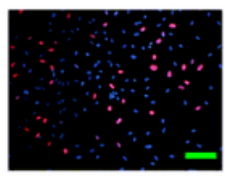

HOXB5-KD1

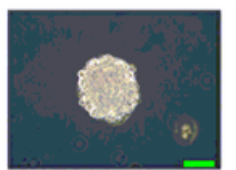

HOXB5-OE

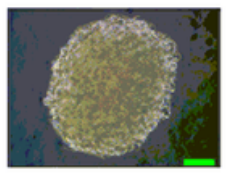

HOXB5-KD2

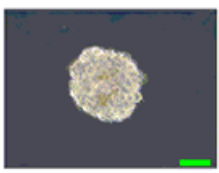

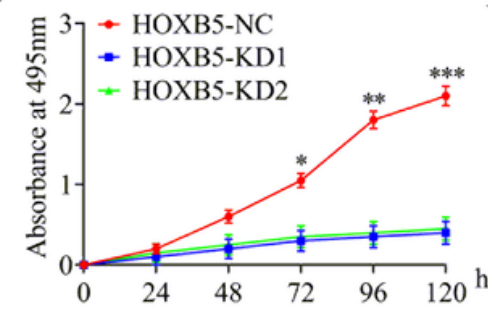

HOXB5-NC

- HOXB5-KD1 HOXB5-EV

[ HOXB5-KD2 HOXB5-OE

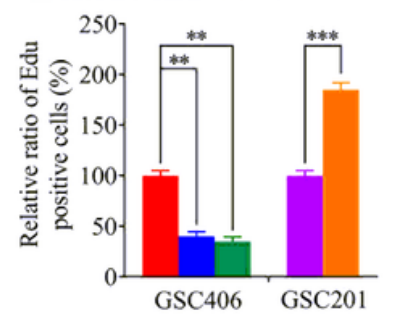

- HOXB5-NC

- HOXB5-KD1 HOXB5-EV

- HOXB5-KD2 $\square$ HOXB5-OE

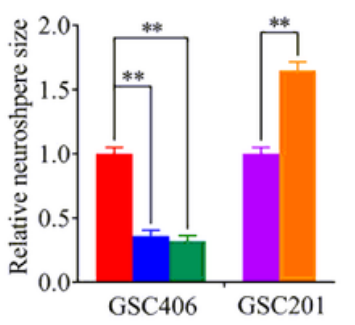

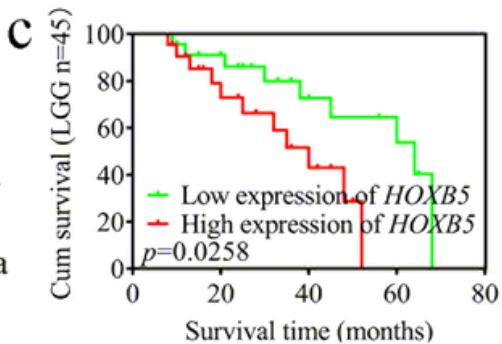

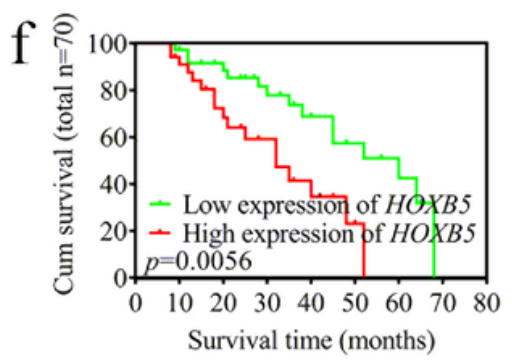

$\mathrm{h}$

GSC201
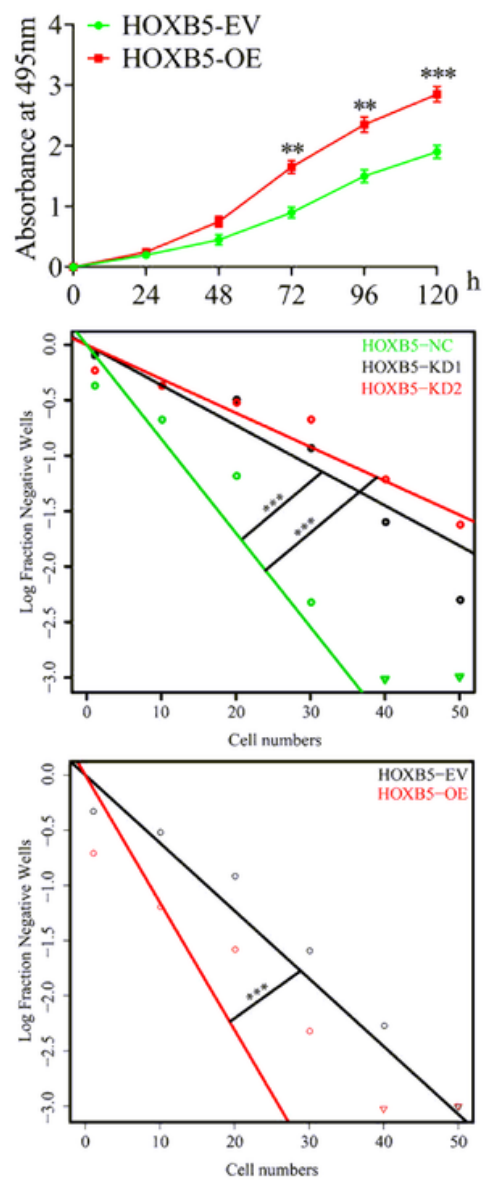

Figure 2

HOXB5 was overexpressed in glioma and correlated with poor patient survival, and regulated the proliferation of GSCs. a, b, d HOXB5 was expressed at higher levels in different grade glioma tissues, compared with normal brain tissues as detected by qRT-PCR (a), western blotting (b), and immunohistochemistry (d). Scale bar $=50 \mu \mathrm{m}$. c, e, f Kaplan-Meier analysis showed the prognostic significance of the 70 glioma patients, 45 LGG patients, and 25 GBM patients with high versus low HOXB5 expressions detected by qRT-PCR. $g$ and $\mathrm{h}$ MTS assays showed that HOXB5 knockdown or 
overexpression affected the cell viability of GSCs. i The EDU assays showed that HOXB5 knockdown or overexpression affected the proliferation of GSCs. Scale bar $=50 \mu \mathrm{m}$. $\mathrm{k}$ The neurospheres formation assays showed that HOXB5 knockdown or overexpression affected the self-renewing capacity of GSCs. Scale bar $=20 \mu \mathrm{m}$. j and I Limiting dilution assays showed that HOXB5 knockdown or overexpression affected the tumor formation incidence of GSCs. All data were expressed as the mean \pm SD (three independent experiments). ${ }^{*} \mathrm{p}<0.05 ;{ }^{* *} \mathrm{p}<0.01 ;{ }^{* * *} \mathrm{p}<0.001$.

a
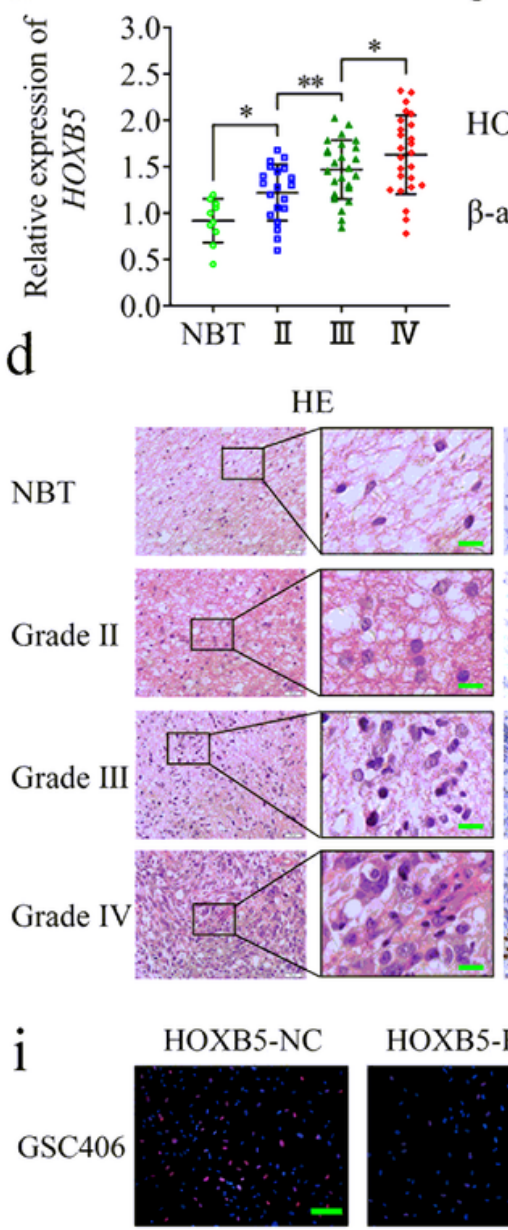

HOXB5-EV

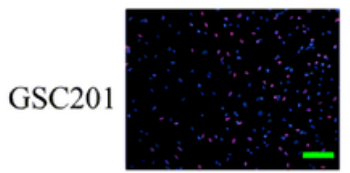

$\mathrm{k}$

GSC406
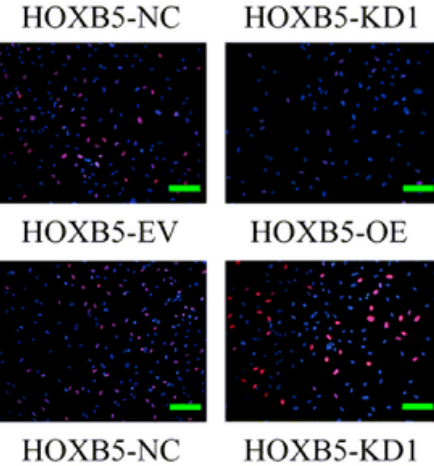

HOXB5-OE

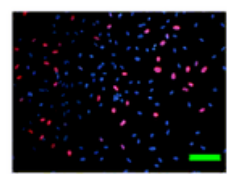

HOXB5-KD1

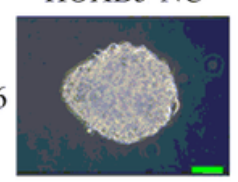

HOXB5-EV

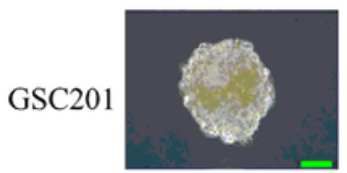

HOXB5-OE

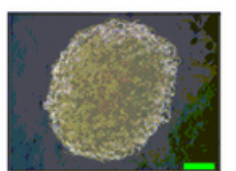

HOXB5-KD2

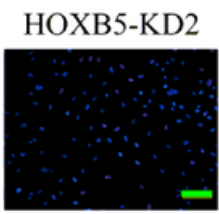

$\mathrm{e}$

\section{$\mathrm{g}$}

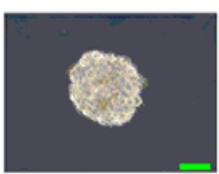

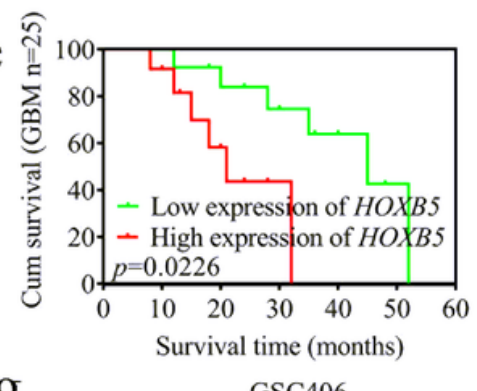

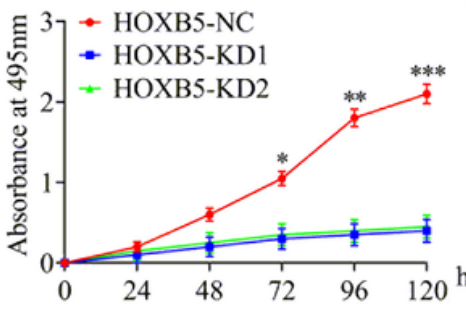

HOXB5-NC

- HOXB5-KD1 HOXB5-EV

- HOXB5-KD2 HOXB5-OE

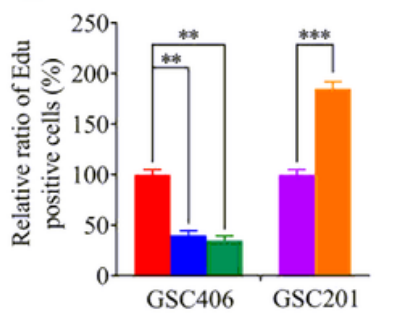

c

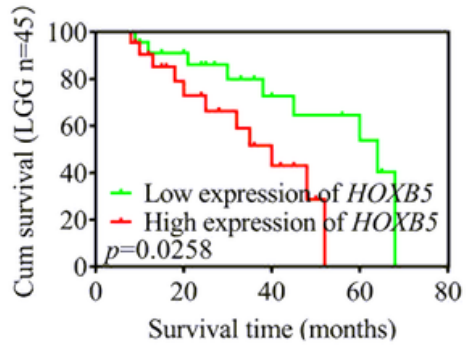

Survival time (months)

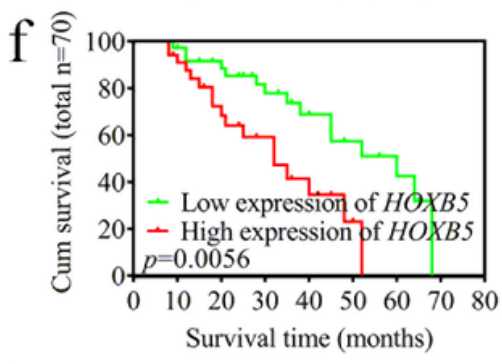

h GSC201

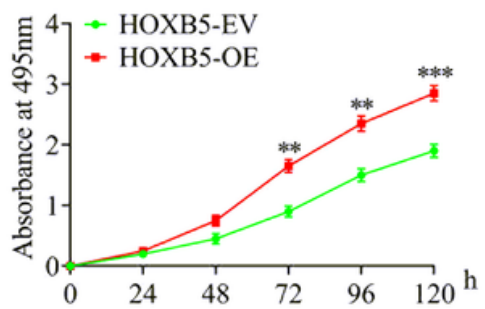

j

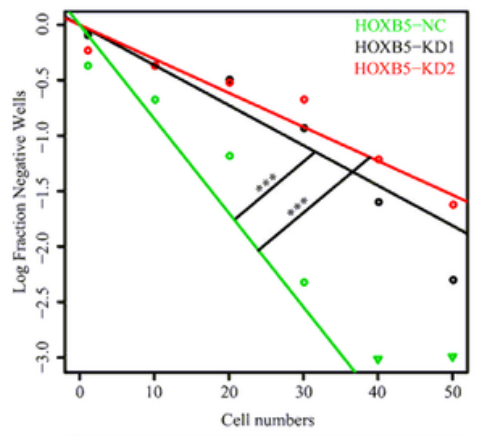

HOXB5-NC

HOXB5-KD1 HOXB5-EV HOXB5-KD2 $=$ HOXB5-OE
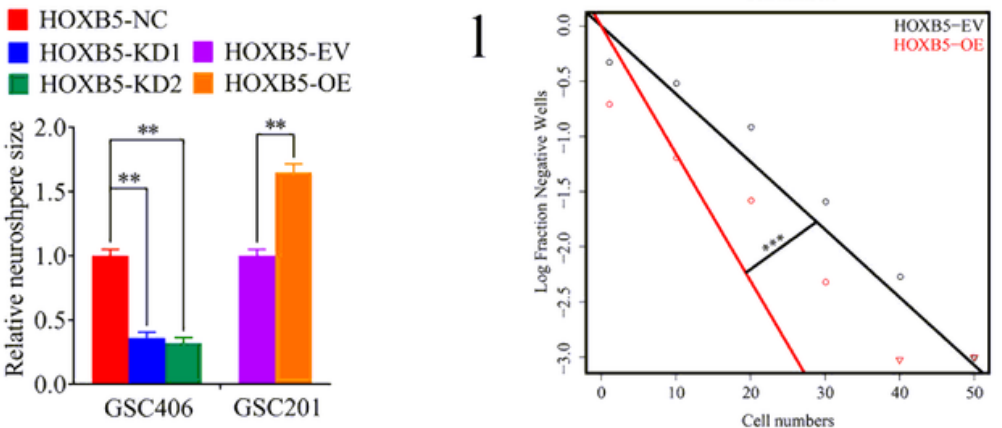

Figure 2 
HOXB5 was overexpressed in glioma and correlated with poor patient survival, and regulated the proliferation of GSCs. a, b, d HOXB5 was expressed at higher levels in different grade glioma tissues, compared with normal brain tissues as detected by qRT-PCR (a), western blotting (b), and immunohistochemistry (d). Scale bar $=50 \mu \mathrm{m}$. c, e, f Kaplan-Meier analysis showed the prognostic significance of the 70 glioma patients, 45 LGG patients, and 25 GBM patients with high versus low HOXB5 expressions detected by qRT-PCR. $g$ and $\mathrm{h}$ MTS assays showed that HOXB5 knockdown or overexpression affected the cell viability of GSCs. i The EDU assays showed that HOXB5 knockdown or overexpression affected the proliferation of GSCs. Scale bar $=50 \mu \mathrm{m}$. $\mathrm{k}$ The neurospheres formation assays showed that HOXB5 knockdown or overexpression affected the self-renewing capacity of GSCs. Scale bar $=20 \mu \mathrm{m}$. $\mathrm{j}$ and I Limiting dilution assays showed that HOXB5 knockdown or overexpression affected the tumor formation incidence of GSCs. All data were expressed as the mean \pm SD (three independent experiments). ${ }^{*} p<0.05 ;{ }^{* *} p<0.01 ;{ }^{* *} p<0.001$.

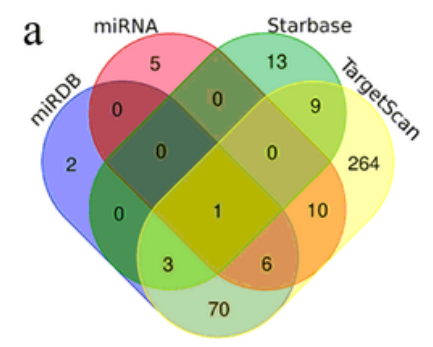

e

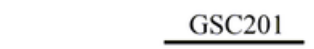

miR-185-5p-NC

miR-185-5p-inhibitor

HOXB5

$\beta$-actin

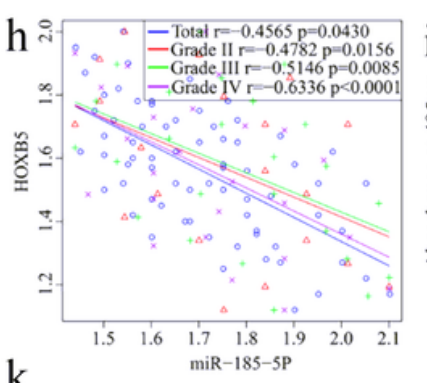

K

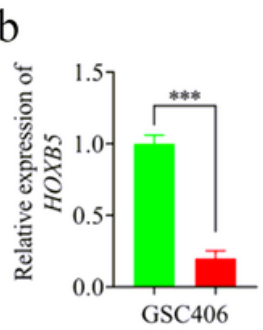

$\mathrm{f}_{\text {HOXB5 }}^{\text {hsa-miR-185-5p }} 3^{\prime}$

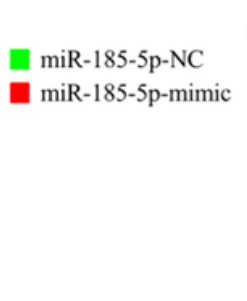

AGUCCUUGACGGAAAGAGAGG c

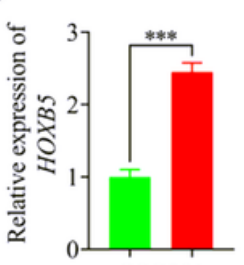

GSC201
C

miR-185-5p-NC
miR-185-5p-inhibi

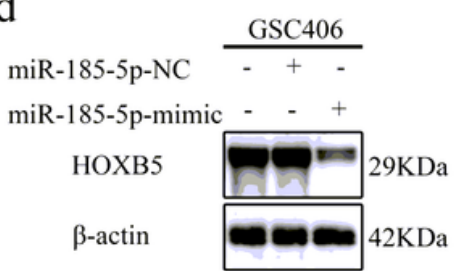

$\mathrm{g}$

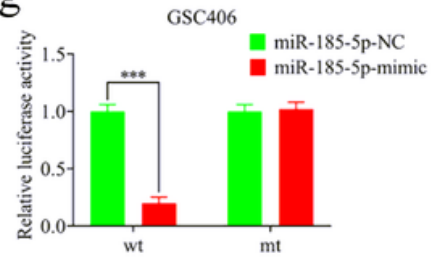

pGL3-HOXB5

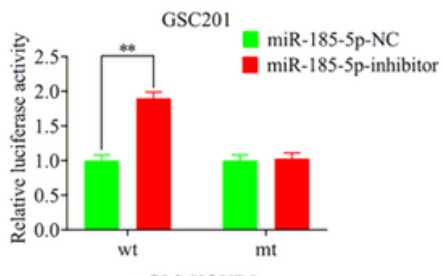

pGL3-HOXB5

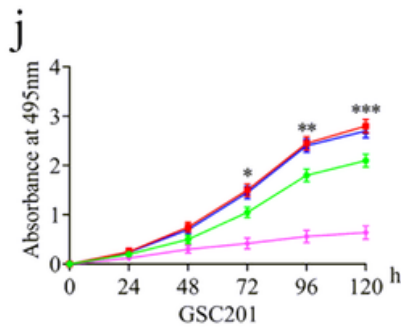

$\mathrm{m}$

$\underset{+}{H O X B 5-E V} \quad$ HOXB5-OE

- miR-185-5p-NC

- miR-185-5p-mimic

- miR-185-5p-mimic+HOXB5-EV

- miR-185-5p-mimic+HOXB5-O
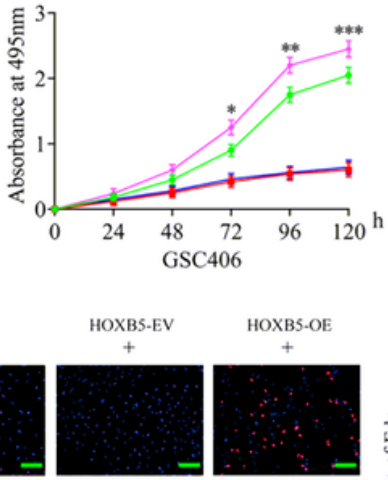

HOXB5-KDI

HOXB5-NC

miR-185-5p-inhibitor

GSC406
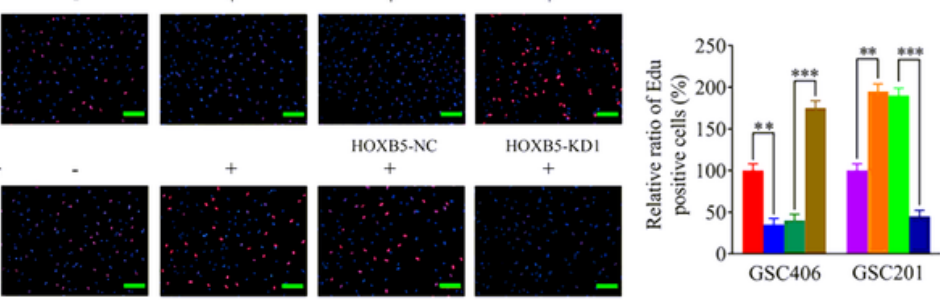

miR-185-5p-NC

miR-185-5p-mimic

miR-185-5p-mimic+HOXB5-EV

miR-185-5p-mimic+HOXB5-OE

miR-185-5p-NC

miR-185-5p-inhibitor

miR-185-5p-inhibitor+HOXB5-NC

miR-185-5p-inhibitor+HOXB5-KDI

HOXB5-EV

HOXBS-OE

miR-185-5p-mimi

GSC406

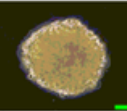

miR-185-Sp-inhibitor

GSC20

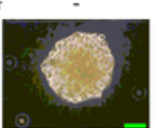

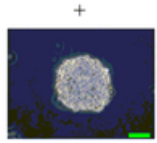

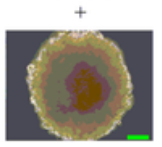

HOXB5-NC

HOXB5-KDI
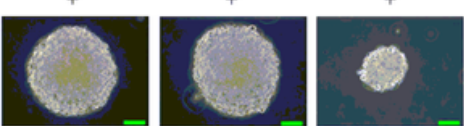
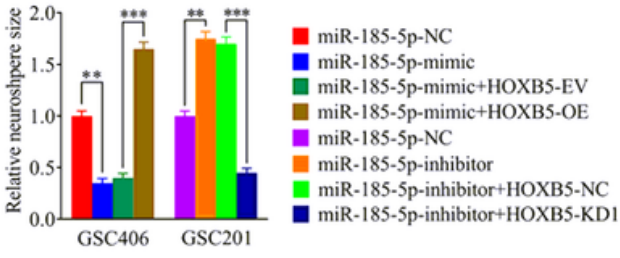

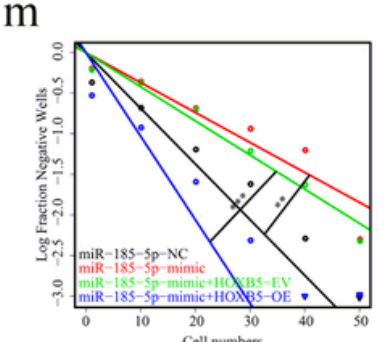

n

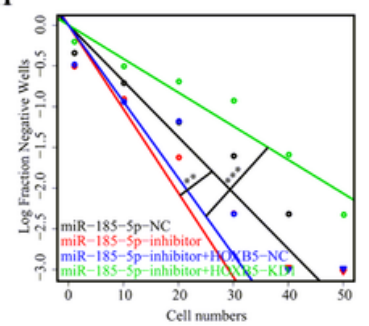




\section{Figure 3}

MiR-185-5p negatively regulated HOXB5 expression and suppressed the proliferation of GSCs. a Identification of a miRNA that potentially regulated HOXB5 expression based on microRNA, miRDB, Starbase, and TargetScan databases. b, c and d, e qRT-PCR (b, c) and western blotting (d, e) showed HOXB5 expression in GSCs after miR-185-5p mimic or inhibitor treatment. f Schematic diagram of the putative miR-185-5p binding site in the 3'-UTR of HOXB5. $g$ The luciferase reporter assays showed that miR-185-5p mimic or inhibitor affected the luciferase activities of HOXB5 in GSCs. $h$ The relative expression correlation between miR-185-5p and HOXB5 in 70 cases of glioma patients were detected by qRT-PCR. $i$ and $j$ MTS assays showed that miR-185-5p mimic or inhibitor treatment affected the cell viability of GSCs and was reversed by HOXB5 overexpression or knockdown, respectively. $k$ The EDU assays showed that miR-185-5p mimic or inhibitor treatment affected the proliferation of GSCs and was reversed by HOXB5 overexpression or knockdown, respectively. Scale bar $=50 \mu \mathrm{m}$. I The neurospheres formation assays showed that miR-185-5p mimic or inhibitor treatment affected the self-renewing capacity of GSCs and was reversed by HOXB5 overexpression or knockdown, respectively. Scale bar = $20 \mu \mathrm{m}$. $\mathrm{m}$ and $\mathrm{n}$ Limiting dilution assays showed that miR-185-5p mimic or inhibitor treatment affected the tumor formation incidence of GSCs and was reversed by HOXB5 overexpression or knockdown, respectively. All data were expressed as the mean $\pm S D$ (three independent experiments). ${ }^{*} p<0.05$; ${ }^{\star *} p<0.01 ; * \star * p<0.001$. 


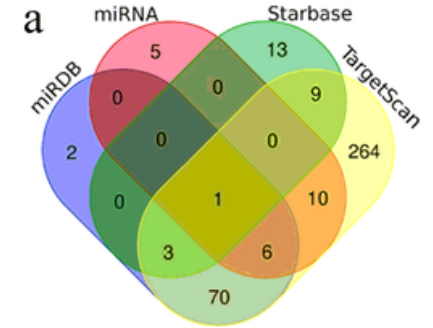

$\mathrm{e}$

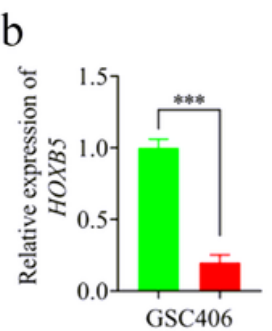

miR-185-5p-NC

miR-185-5p-inhibitor

HOXB5

$\beta$-actin

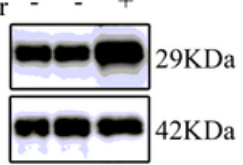

f
HохB5
miR-185-5p-NC
miR-185-5p-mimic

c

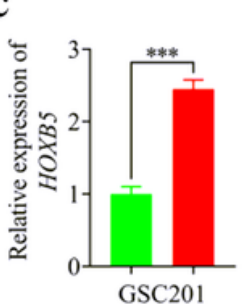

d

miR-185-5p-NC

miR-185-5p-inhibitor

miR-185-5p-NC $\frac{\text { GSC406 }}{}$

miR-185-5p-mimic

HOXB5

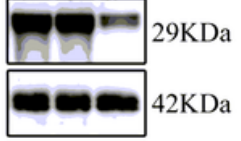

$\beta$-actin

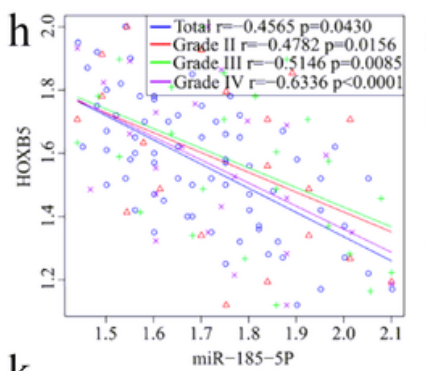

K

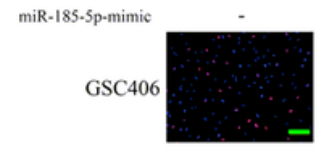

GSC201

1

miR-185-5p-mimic GSC406
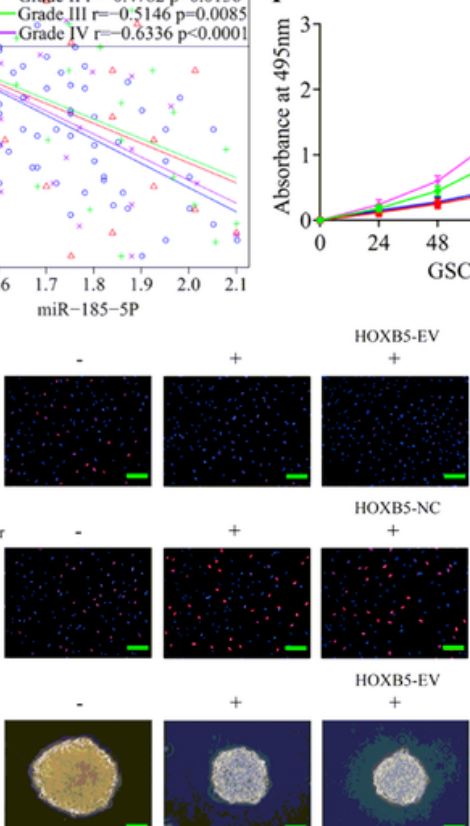

HOXB5-EV

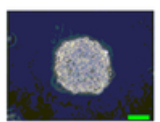
GSC201

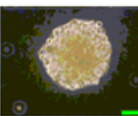

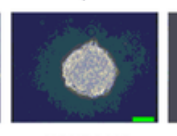

нохB5-NC

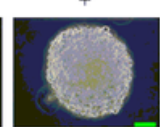

HOXB5-OE

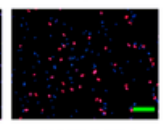

HOXB5-KDI

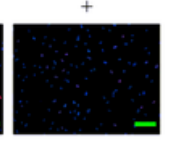

HOXB5-OE

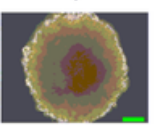

HOXBS-KDI

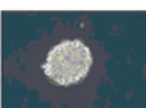

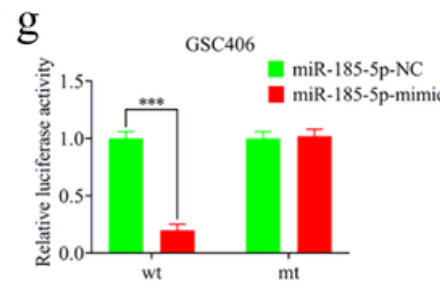

pGL3-HOXB5

j
- miR-185-5p-NC

- miR-185-5p-mimic

- miR-185-5p-mimic+HOXB5-EV

- miR-185-5p-mimic+HOXB5-OE

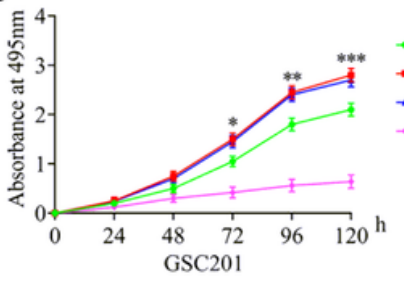

$\mathrm{m}$

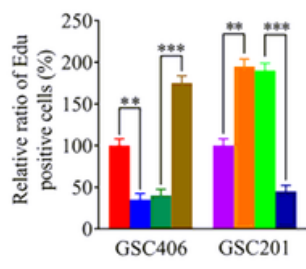

miR-185-5p-NC

IiR-185-5p-mimic

miR-185-5p-mimic+HOXB5-EV

miR-185-5p-mimic+HOXB5-OE

miR-185-5p-NC

miR-185-5p-inhibitor

miR-185-5p-inhibitor+HOXB5-NC

miR-185-5p-inhibitor+HOXB5-KDI

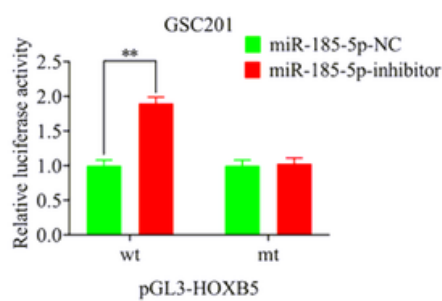

pGL3-HOXB5

- miR-185-5p-NC

- miR-185-5p-inhibitor

- miR-185-5p-inhibitor+HOXB5-NC - miR-185-5p-inhibitor+HOXB5-KD1

\section{Figure 3}

MiR-185-5p negatively regulated HOXB5 expression and suppressed the proliferation of GSCs. a Identification of a miRNA that potentially regulated HOXB5 expression based on microRNA, miRDB, Starbase, and TargetScan databases. b, c and d, e qRT-PCR (b, c) and western blotting (d, e) showed HOXB5 expression in GSCs after miR-185-5p mimic or inhibitor treatment. $f$ Schematic diagram of the putative miR-185-5p binding site in the 3'-UTR of HOXB5. $g$ The luciferase reporter assays showed that miR-185-5p mimic or inhibitor affected the luciferase activities of HOXB5 in GSCs. $h$ The relative expression correlation between miR-185-5p and HOXB5 in 70 cases of glioma patients were detected by qRT-PCR. $i$ and $j$ MTS assays showed that miR-185-5p mimic or inhibitor treatment affected the cell viability of GSCs and was reversed by HOXB5 overexpression or knockdown, respectively. $k$ The EDU assays showed that miR-185-5p mimic or inhibitor treatment affected the proliferation of GSCs and was 
reversed by HOXB5 overexpression or knockdown, respectively. Scale bar $=50 \mu \mathrm{m}$. I The neurospheres formation assays showed that miR-185-5p mimic or inhibitor treatment affected the self-renewing capacity of GSCs and was reversed by HOXB5 overexpression or knockdown, respectively. Scale bar = $20 \mu \mathrm{m}$. $\mathrm{m}$ and $\mathrm{n}$ Limiting dilution assays showed that miR-185-5p mimic or inhibitor treatment affected the tumor formation incidence of GSCs and was reversed by HOXB5 overexpression or knockdown, respectively. All data were expressed as the mean \pm SD (three independent experiments). ${ }^{*}<<0.05$; ${ }^{* *} p<0.01 ; * * * p<0.001$.

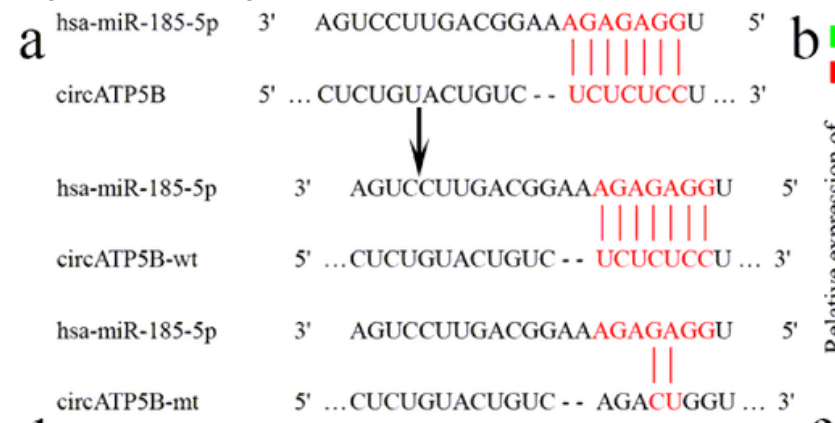

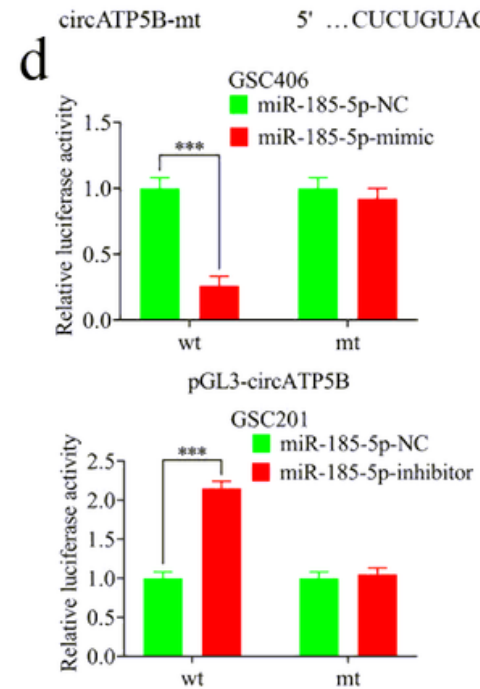

h
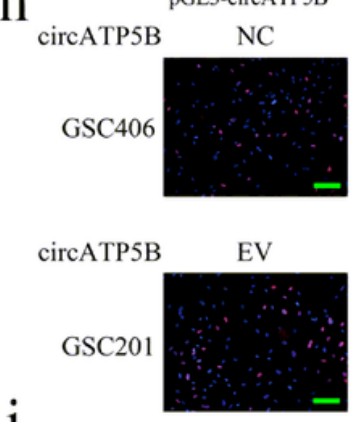

J

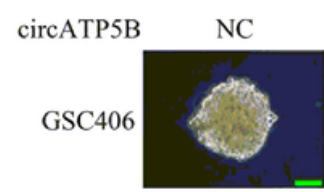

circATP5B

EV

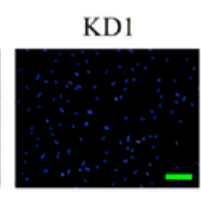

OE

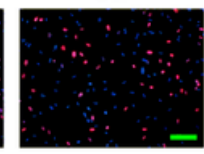

KD1

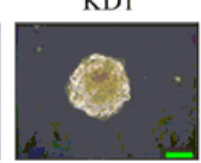

$\mathrm{OE}$
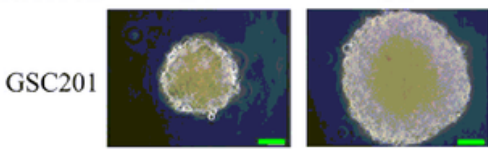

e

e $\begin{gathered}\text { GSC406 } \\ \text { miR-185-5p-NC } \\ \text { miR-185-5p-mimic }\end{gathered}$

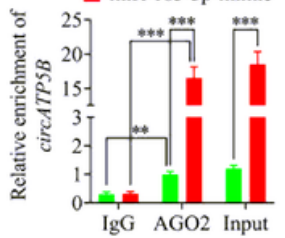

GSC406

miR-185-5p-NC

miR-185-5p-mimic

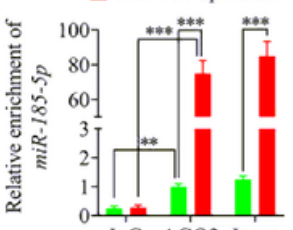

IgG $\mathrm{AGO} 2$ Input

miR-185-5p-NC miR-185-5p-inhibitor

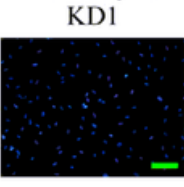

$\mathrm{OE}$

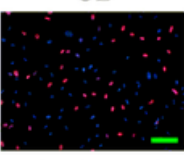

miR-185-5p-NC

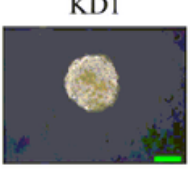

miR-185-5p-NC

$\mathrm{OE}$
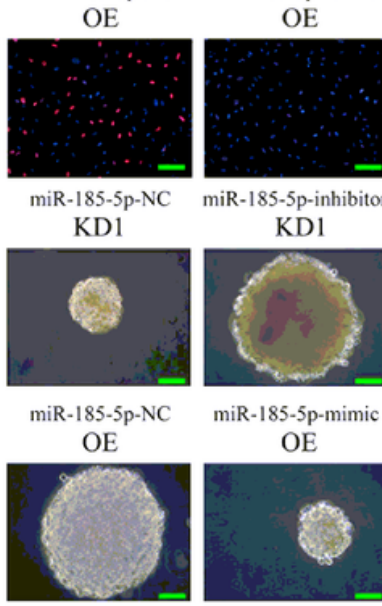

KD1

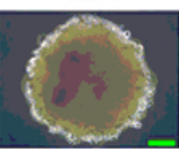

miR-185-5p-mimic

$\mathrm{OE}$

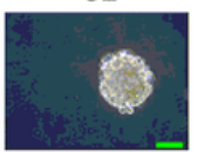

$\mathrm{O}$ miR-185-5p-NC
miR-185-5p-mimic

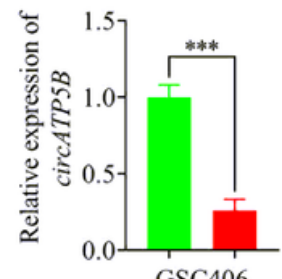

GSC406

\section{- circATP5B-NC}

- circATP5B-KD1

- circATP5B-KD1+miR-185-5p-NC

- circATP5B-KD1+miR-185-5p-inhibitor

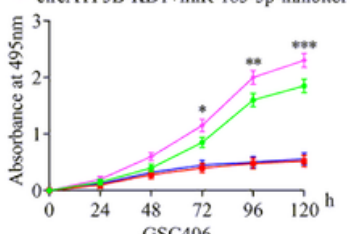

- circATP5B-EV

- circATP5B-OE

- circATP5B-OE+miR-185-5p-NC

- circATP5B-OE+miR-185-5p-mimic
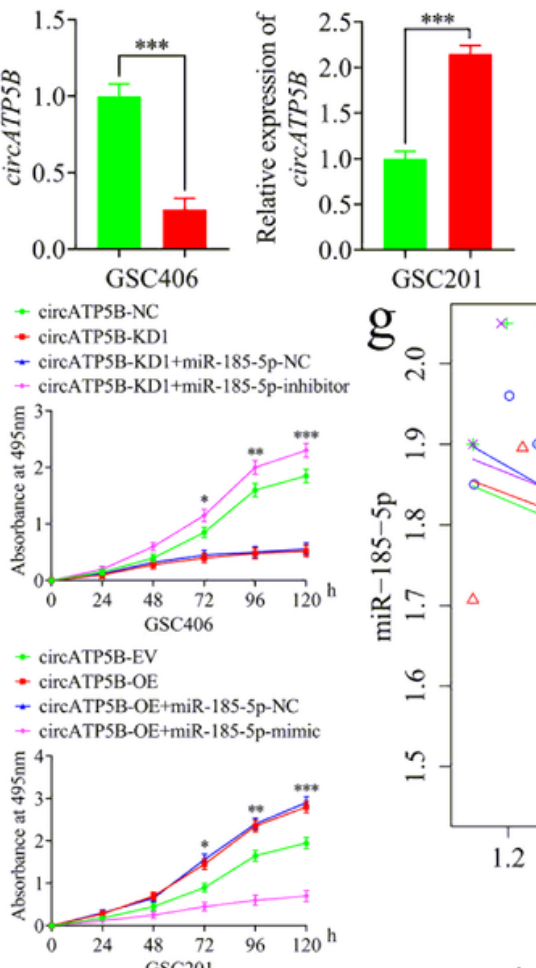

GSC201

circATP5B-KD

circATP5B-KD2

circATP5B-EV

circATP5B-OE
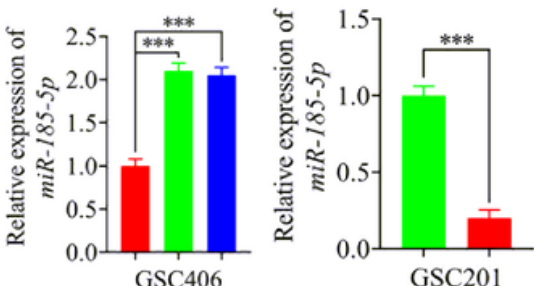

GSC406

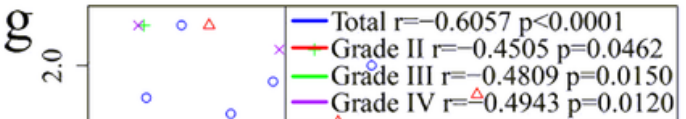

GSC201

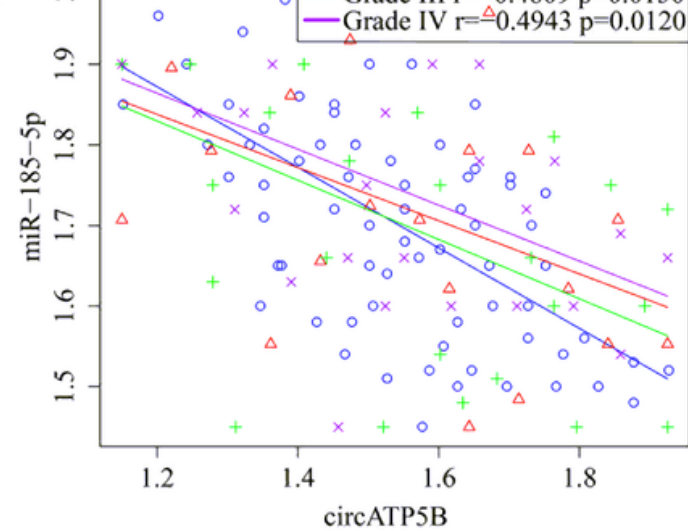

circATP5B-NC

cirCATPSB-KDI

circATP5B-KD1+miR-185-5p-NC

circATPSB-KDI+miR-185-5p-inhibitor

circATP5B-EV

circATP5B-OE

circATP5B-OE+miR-185-5p-NC

- circATP5B-OE+miR-185-5p-mimic
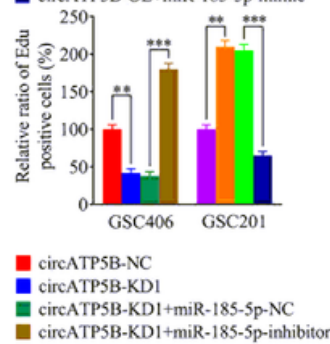

= circATPSB-KDI+miR-185-5p-NC

circATP5B-KD

= circATPSB-OE

circATP5B-OE+miR-185-5p-NC

- circATPSB-OE+miR-185-5p-mimic

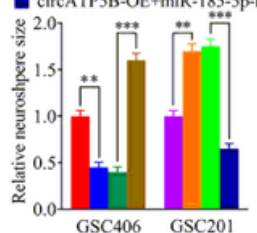

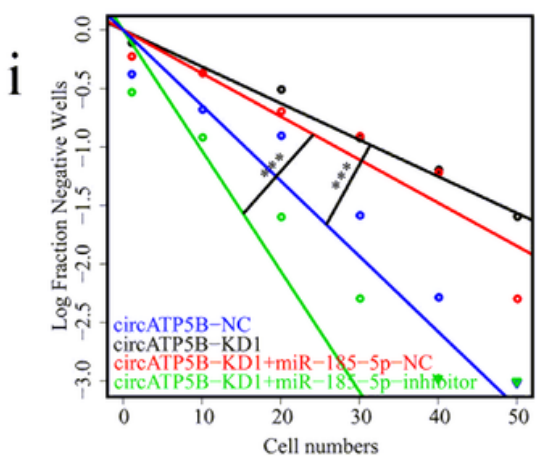

$\mathrm{k}$

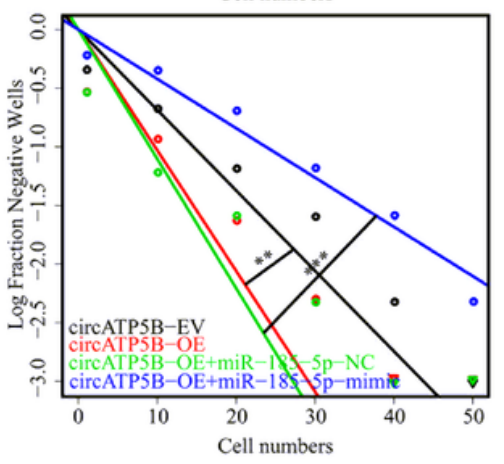

Figure 4 
circATP5B promoted the proliferation of GSCs through miRNA sponging of miR-185-5p. a Graphical illustration showing the predicted position of the circATP5B target on the miR-185-5p sequence. $b$ and $c$ qRT-PCR showed the relative expression of circATP5B in GSCs after miR-185-5p mimic or inhibitor treatment (b), and the relative expression of miR-185-5p in GSCs following circATP5B knockdown or overexpression (c). $d$ The luciferase reporter assays showed that miR-185-5p mimic or inhibitor affected the luciferase activities of circATP5B in GSCs. e The RNA immunoprecipitation (RIP) assay was performed in GSC406 after the miR-185-5p mimic or negative control was transfected, followed by qRTPCR to detect the enrichment of circATP5B and miR-185-5p. f MTS assays showed that circATP5B knockdown or overexpression affected the cell viability of GSCs and was reversed by the miR-185-5p inhibitor or mimic treatment, respectively. $g$ The relative expression correlation between circATP5B and miR-185-5p in 70 cases of glioma patients were detected by qRT-PCR. $\mathrm{h}$ The EDU assays showed that circATP5B knockdown or overexpression affected the proliferation of GSCs and was reversed by miR-185$5 p$ inhibitor or mimic treatment, respectively. Scale bar $=50 \mu \mathrm{m}$. $j$ The neurospheres formation assays showed that circATP5B knockdown or overexpression affected the self-renewing capacity of GSCs and was reversed by miR-185-5p inhibitor or mimic treatment, respectively. Scale bar $=20 \mu \mathrm{m}$. i and $\mathrm{k}$ Limiting dilution assays showed that circATP5B knockdown or overexpression affected the tumor formation incidence of GSCs and was reversed by miR-185-5p inhibitor or mimic treatment, respectively. All data were expressed as the mean \pm SD (three independent experiments). ${ }^{*} p<0.05 ;{ }^{* \star} p<0.01 ;{ }^{* \star \star} p<0.001$. 
hsa-miR-185-5p 3' AGUCCUUGACGGAAAGAGAGGU

circATP5B $\quad 5^{\prime}$... CUCUGUACUGUC .. UCUCUCCU ... $3^{\prime}$

$\mathrm{O}=\mathrm{miR}-185-5 \mathrm{p}-\mathrm{NC}$
$\mathrm{miR}-185-5 \mathrm{p}-\mathrm{mimic}$

miR-185-5p-NC C circATP5B-NC

miR-185-5p-inhibitor

circATP5B-KD1

circATP5B-OE

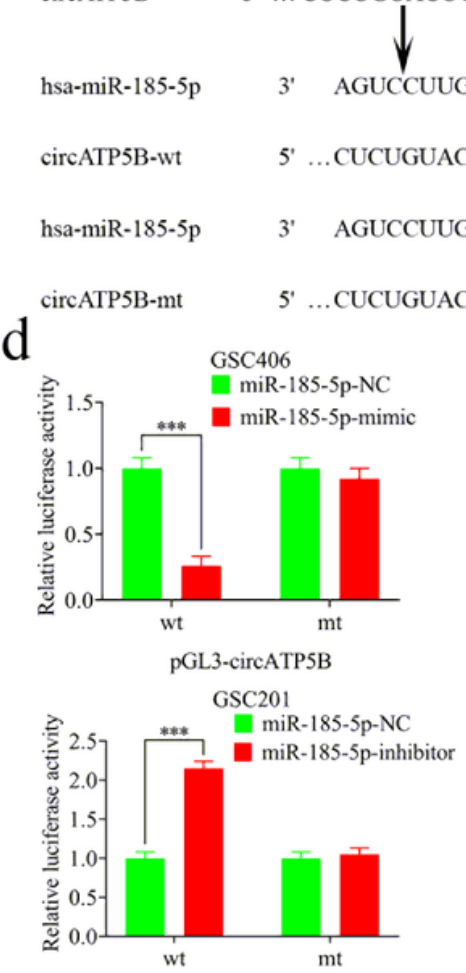

$\mathrm{h}$

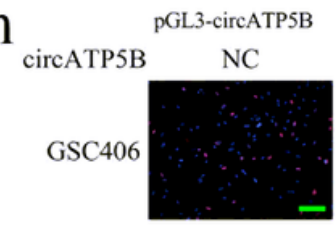

circATP5B

EV

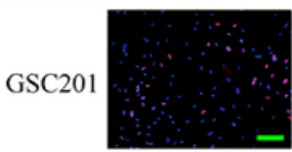

j

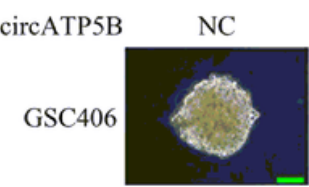

circATP5B

EV
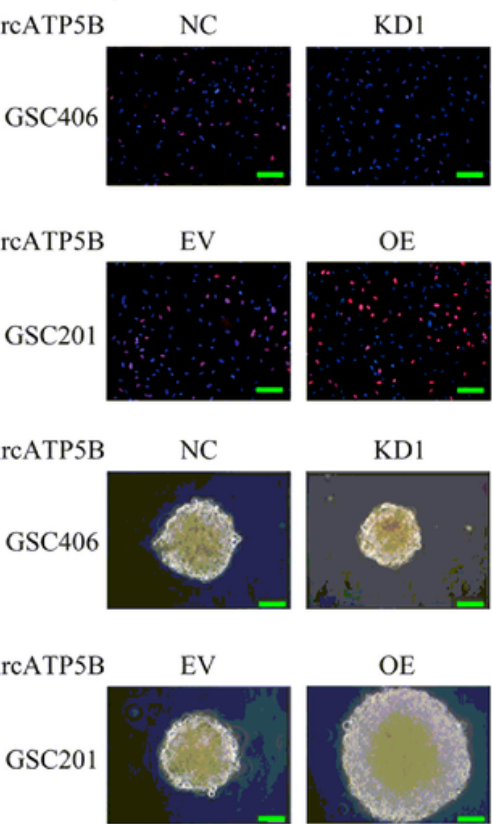

e

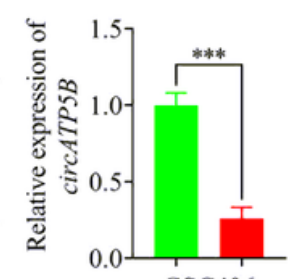

GSC406

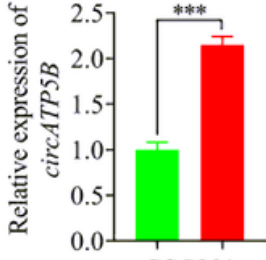

GSC201

circATP5B-KD2

\section{$\rightarrow$ circATP5B-NC}

- circATP5B-KD1

- circATP5B-KD1+miR-185-5p-NC

- circATP5B-KD1+miR-185-5p-NC
- circATP5B-KD1+miR-185-5p-inhibitor
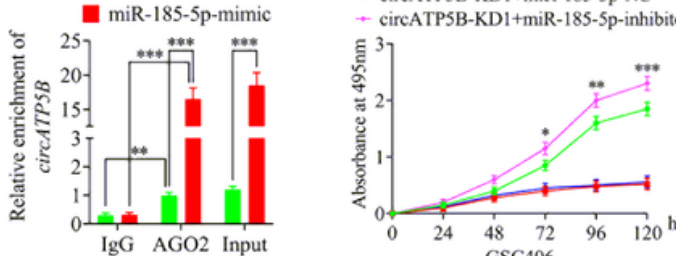

GSC406

miR-185-5p-NC

miR-185-5p-mimic

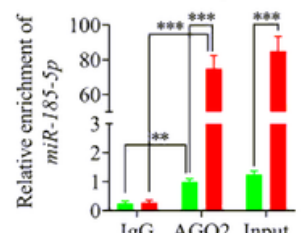

- circATP5B-EV
- circATP5B-OE
- circATP5B-OE+miR-185-5p-NC

- circATP5B-OE+miR-185-5p-mimic

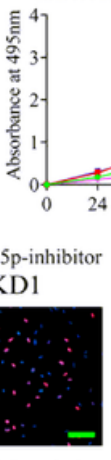

miR-185-5p-NC miR-185-5p-inhibitor

$\mathrm{OE}$

KD1

$\mathrm{OE}$

KD1

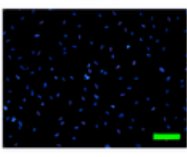

$\mathrm{OE}$

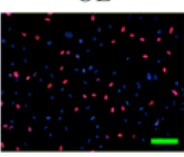

miR-185-5p-NC

KD1

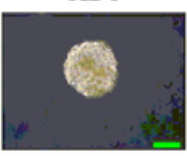

miR-185-5p-NC

OE
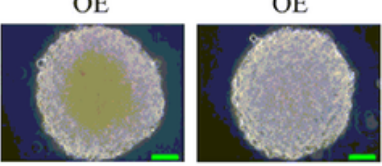

$\mathrm{OE}$

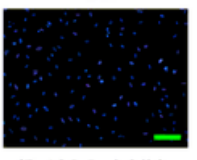

KD1

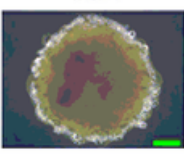

miR-185-5p-mimic

$\mathrm{OE}$

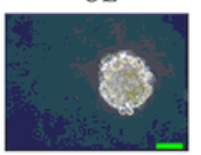

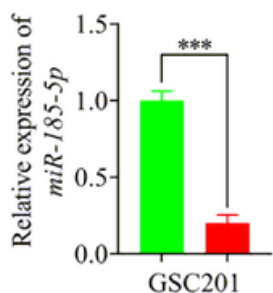
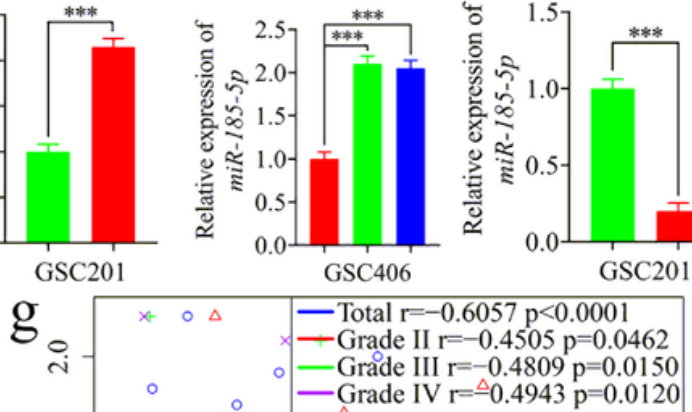

GSC406

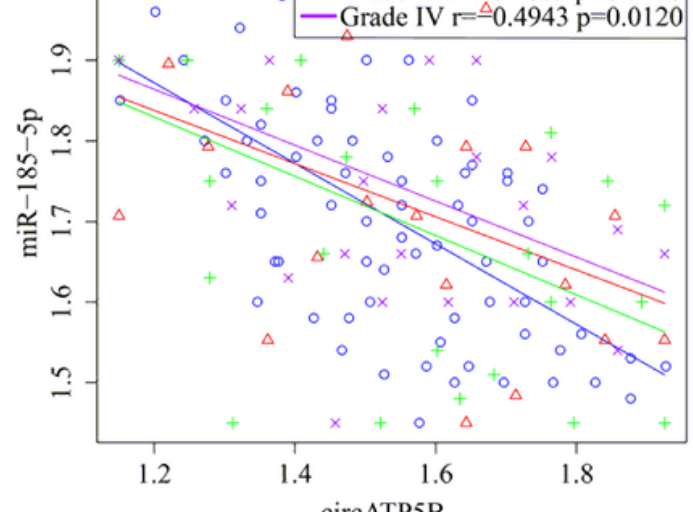

circATP5B

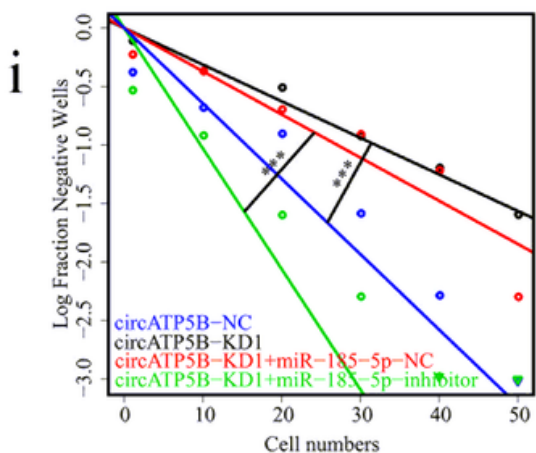

k

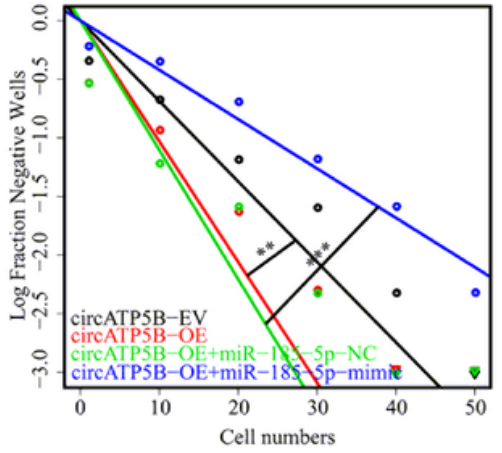

Figure 4

circATP5B promoted the proliferation of GSCs through miRNA sponging of miR-185-5p. a Graphical illustration showing the predicted position of the circATP5B target on the miR-185-5p sequence. $b$ and $c$ qRT-PCR showed the relative expression of circATP5B in GSCs after miR-185-5p mimic or inhibitor treatment (b), and the relative expression of miR-185-5p in GSCs following circATP5B knockdown or overexpression (c). d The luciferase reporter assays showed that miR-185-5p mimic or inhibitor affected the luciferase activities of circATP5B in GSCs. e The RNA immunoprecipitation (RIP) assay was 

performed in GSC406 after the miR-185-5p mimic or negative control was transfected, followed by qRT-
PCR to detect the enrichment of circATP5B and miR-185-5p. f MTS assays showed that circATP5B
knockdown or overexpression affected the cell viability of GSCs and was reversed by the miR-185-5p
inhibitor or mimic treatment, respectively. $g$ The relative expression correlation between circATP5B and
miR-185-5p in 70 cases of glioma patients were detected by qRT-PCR. $h$ The EDU assays showed that
circATP5B knockdown or overexpression affected the proliferation of GSCs and was reversed by miR-185performed in GSC406 after the miR-185-5p mimic or negative control was transfected, followed by qRT-
PCR to detect the enrichment of circATP5B and miR-185-5p. f MTS assays showed that circATP5B
knockdown or overexpression affected the cell viability of GSCs and was reversed by the miR-185-5p
inhibitor or mimic treatment, respectively. $g$ The relative expression correlation between circATP5B and
miR-185-5p in 70 cases of glioma patients were detected by qRT-PCR. $h$ The EDU assays showed that
circATP5B knockdown or overexpression affected the proliferation of GSCs and was reversed by miR-185performed in GSC406 after the miR-185-5p mimic or negative control was transfected, followed by qRT-
PCR to detect the enrichment of circATP5B and miR-185-5p. f MTS assays showed that circATP5B
knockdown or overexpression affected the cell viability of GSCs and was reversed by the miR-185-5p
inhibitor or mimic treatment, respectively. $g$ The relative expression correlation between circATP5B and
miR-185-5p in 70 cases of glioma patients were detected by qRT-PCR. $h$ The EDU assays showed that
circATP5B knockdown or overexpression affected the proliferation of GSCs and was reversed by miR-185performed in GSC406 after the miR-185-5p mimic or negative control was transfected, followed by qRT-
PCR to detect the enrichment of circATP5B and miR-185-5p. f MTS assays showed that circATP5B
knockdown or overexpression affected the cell viability of GSCs and was reversed by the miR-185-5p
inhibitor or mimic treatment, respectively. $g$ The relative expression correlation between circATP5B and
miR-185-5p in 70 cases of glioma patients were detected by qRT-PCR. $h$ The EDU assays showed that
circATP5B knockdown or overexpression affected the proliferation of GSCs and was reversed by miR-185performed in GSC406 after the miR-185-5p mimic or negative control was transfected, followed by qRT-
PCR to detect the enrichment of circATP5B and miR-185-5p. f MTS assays showed that circATP5B
knockdown or overexpression affected the cell viability of GSCs and was reversed by the miR-185-5p
inhibitor or mimic treatment, respectively. $g$ The relative expression correlation between circATP5B and
miR-185-5p in 70 cases of glioma patients were detected by qRT-PCR. $h$ The EDU assays showed that
circATP5B knockdown or overexpression affected the proliferation of GSCs and was reversed by miR-185performed in GSC406 after the miR-185-5p mimic or negative control was transfected, followed by qRT-
PCR to detect the enrichment of circATP5B and miR-185-5p. f MTS assays showed that circATP5B
knockdown or overexpression affected the cell viability of GSCs and was reversed by the miR-185-5p
inhibitor or mimic treatment, respectively. $g$ The relative expression correlation between circATP5B and
miR-185-5p in 70 cases of glioma patients were detected by qRT-PCR. $h$ The EDU assays showed that
circATP5B knockdown or overexpression affected the proliferation of GSCs and was reversed by miR-185$5 p$ inhibitor or mimic treatment, respectively. Scale bar $=50 \mu \mathrm{m}$. $\mathrm{j}$ The neurospheres formation assays showed that circATP5B knockdown or overexpression affected the self-renewing capacity of GSCs and was reversed by miR-185-5p inhibitor or mimic treatment, respectively. Scale bar $=20 \mu \mathrm{m}$. $\mathrm{i}$ and $\mathrm{k}$ Limiting dilution assays showed that circATP5B knockdown or overexpression affected the tumor formation incidence of GSCs and was reversed by miR-185-5p inhibitor or mimic treatment, respectively. All data were expressed as the mean \pm SD (three independent experiments). ${ }^{*} p<0.05 ;{ }^{* \star} p<0.01 ; * \star \star x p 0.001$.
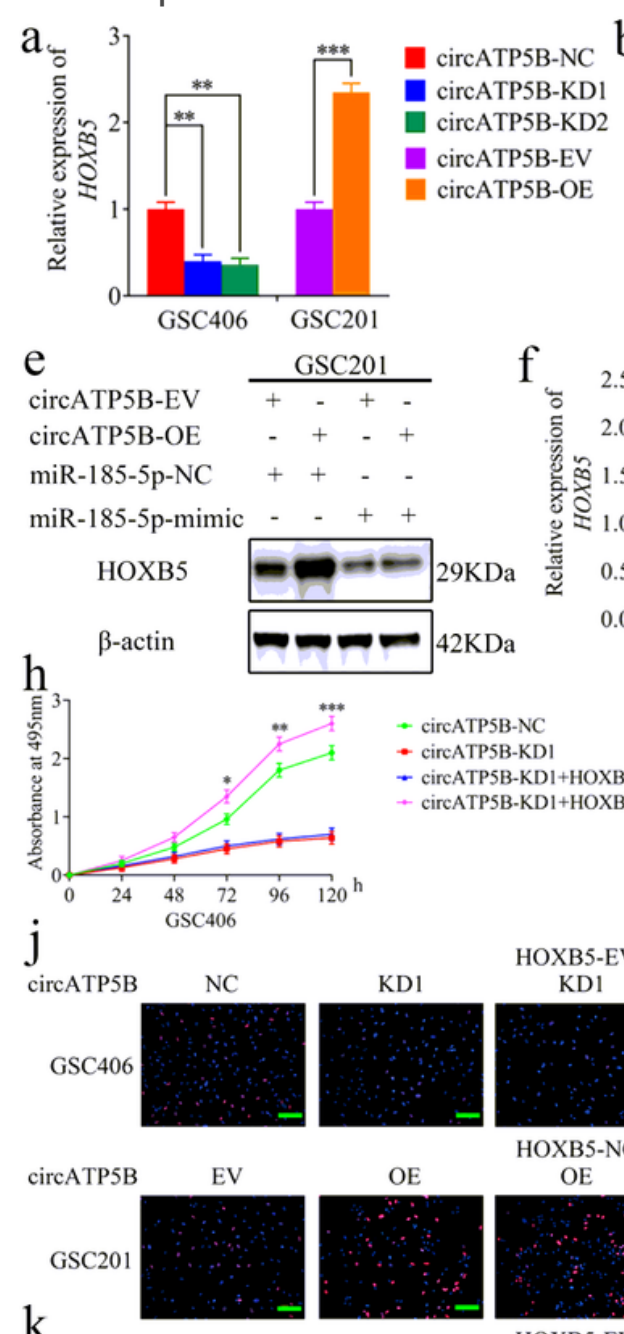

$\mathrm{K}$

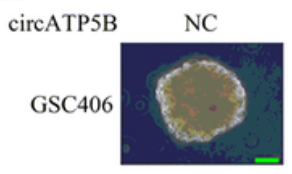

circATP5B

EV

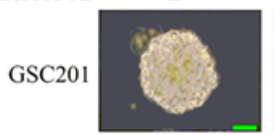

- circATP5B-KDI+HOXB5-EV

- circATP5B-KDI+HOXBS-OE
KD1

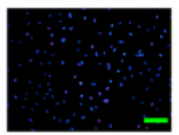

OE

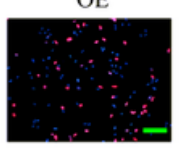
OE

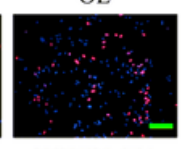

HOXB5-EV

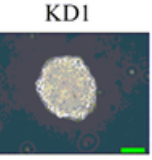

$\mathrm{OE}$

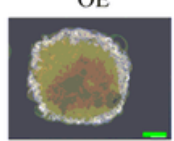

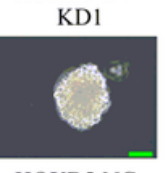

HOXB5-NC

$\mathrm{OE}$

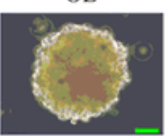

$\mathrm{b}$

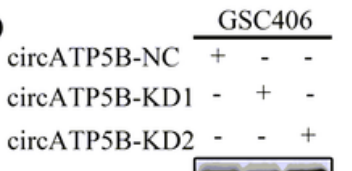

HOXB5

$\beta$-actin

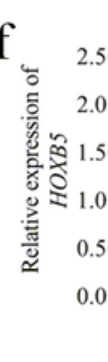

c GSC201
circATP5B-EV +-
circATP5B-OE - +

HOXB5

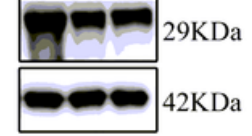

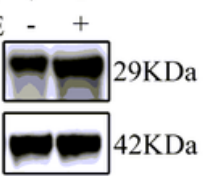

d circATP5B-NC circATP5B-KD1 miR-185-5p-NC miR-185-5p-inhibitor

HOXB5

$\beta$-actin

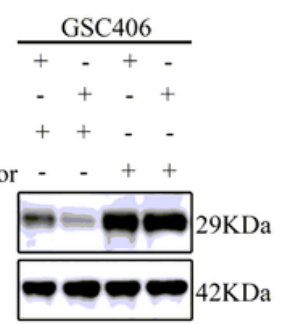

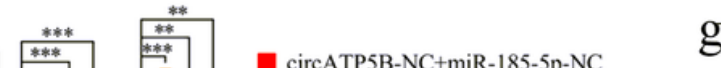

g circATP5B-KD1+miR-185-5p-NC circATP5B-NC+miR-185-5p-inhibitor circATP5B-KD1+miR-185-5p-inhibitor circATP5B-EV+miR-185-5p-NC circATP5B-OE+miR-185-5p-NC circATP5B-EV+miR-185-5p-mimic circATP5B-OE+miR-185-5p-mimic

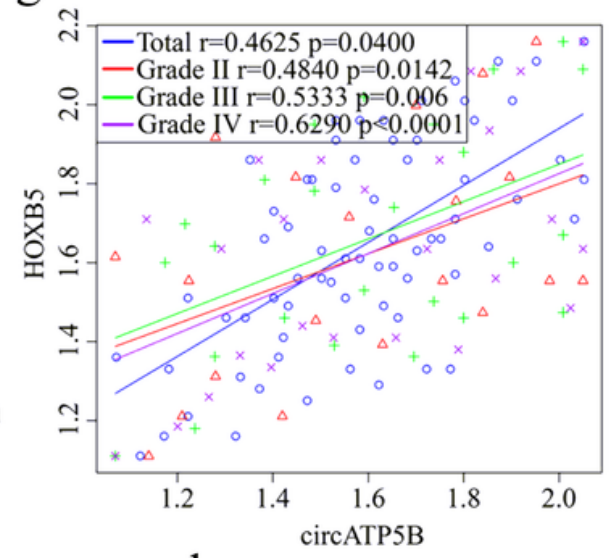
HOXB5-EV HOXB5-OE

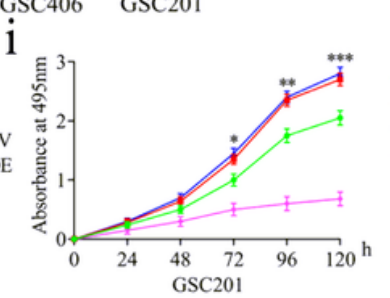

- circATPSB-EV

- circATP5B-OE

- circATP5B-OE+HOXB5-NC - circATPSB-OE+HOXBS-KDI

KD1

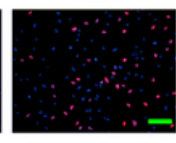

HOXB5-KD1

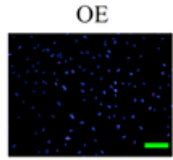

HOXB5-OE

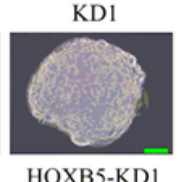

$\mathrm{OE}$

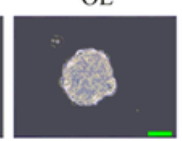

1

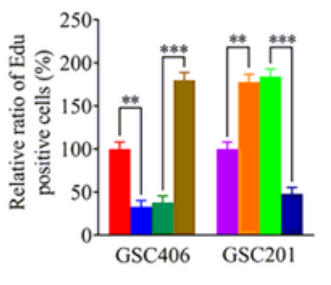

- circATP5B-NC

a circATP5B-KDI

circATP5B-KDI+HOXB5-EV

circATP5B-KD1+HOXB5-OE

circATP5B-EV

circATP5B-OE

circATP5B-OE+HOXB5-NC

circATP5B-OE+HOXB5-KDI
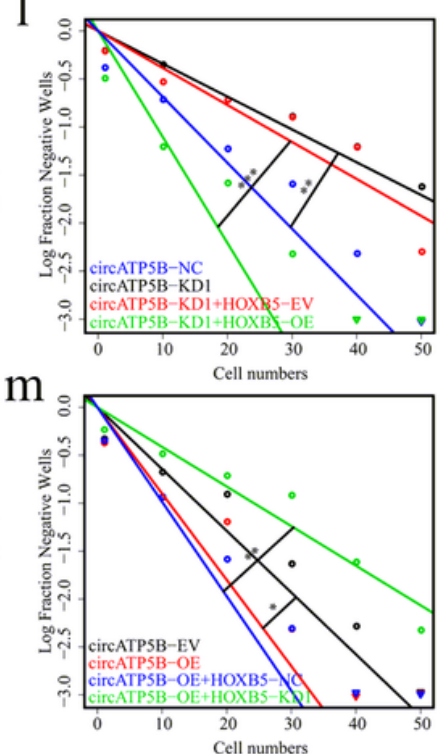

circATP5B-NC

circATP5B-KD1

circATP5B-KD1+HOXB5-EV

circATP5B-KD1+HOXB5-O

circATP5B-EV

circATP5B-OE

circATP5B-OE+HOXB5-NC

a circATP5B-OE+HOXB5-KD1 performed in GSC406 after the miR-185-5p mimic or negative control was transfected, followed by qRT-
PCR to detect the enrichment of circATP5B and miR-185-5p. $\mathrm{f}$ MTS assays showed that circATP5B
knockdown or overexpression affected the cell viability of GSCs and was reversed by the miR-185-5p
inhibitor or mimic treatment, respectively. $g$ The relative expression correlation between circATP5B and
miR-185-5p in 70 cases of glioma patients were detected by qRT-PCR. $\mathrm{h}$ The EDU assays showed that
circATP5B knockdown or overexpression affected the proliferation of GSCs and was reversed by miR-185- 


\section{Figure 5}

CircATP5B promoted the proliferation of GSCs by upregulating HOXB5 expression. a, b, c qRT-PCR (a) and western blotting $(b, c)$ showed the expression of HOXB5 in GSCs after circATP5B knockdown or overexpression. $d$ The decreased expression of HOXB5 in GSC406 induced by circATP5B knockdown was reversed by miR-185-5p inhibitor treatment, as detected by western blotting. e The increased expression of HOXB5 in GSC201 induced by circATP5B overexpression was reversed by miR-185-5p mimic treatment, as determined by western blotting. $f$ The effects of both circATP5B and miR-185-5p on the expression of HOXB5 in GSCs were detected by qRT-PCR. $g$ The relative expression correlation between circATP5B and HOXB5 in 70 cases of glioma patients were detected by qRT-PCR. $h$ and i MTS assays showed that circATP5B knockdown or overexpression affected the cell viability of GSCs and was reversed by HOXB5 overexpression or knockdown, respectively. j The EDU assays showed that circATP5B knockdown or overexpression affected the proliferation of GSCs and was reversed by HOXB5 overexpression or knockdown, respectively. Scale bar $=50 \mu \mathrm{m}$. $\mathrm{k}$ The neurospheres formation assays showed that circATP5B knockdown or overexpression affected the self-renewing capacity of GSCs and was reversed by HOXB5 overexpression or knockdown, respectively. Scale bar $=20 \mu \mathrm{m}$. I and $\mathrm{m}$ Limiting dilution assays showed that circATP5B knockdown or overexpression affected the tumor formation incidence of GSCs and was reversed by HOXB5 overexpression or knockdown, respectively. All data were expressed as the mean \pm SD (three independent experiments). ${ }^{*} p<0.05 ;{ }^{* \star} p<0.01 ;{ }^{* \star *} p<0.001$. 


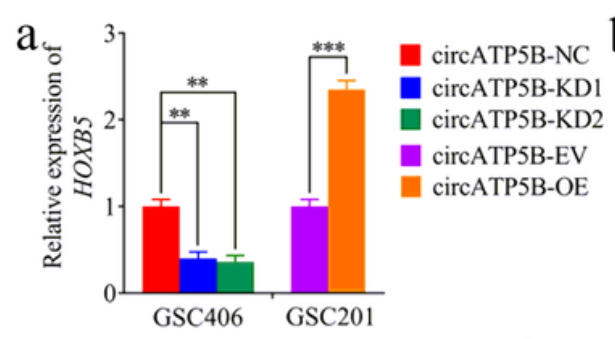

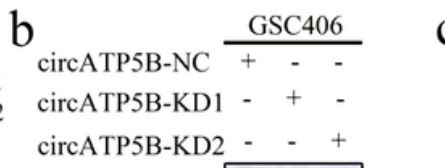

$\mathrm{C}$

HOXB5

$\beta$-actin
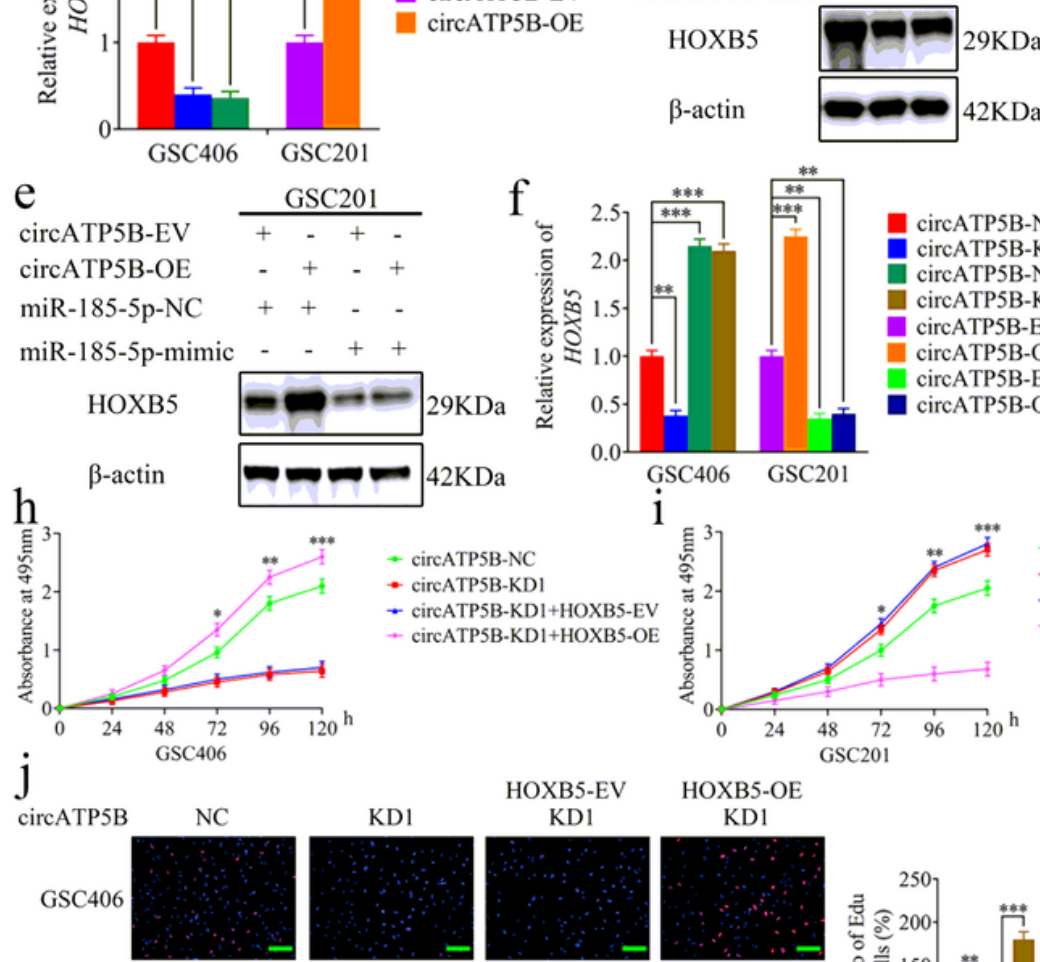

circATP5B-EV

circATP5B-OE

miR-185-5p-NC

miR-185-5p-mimic
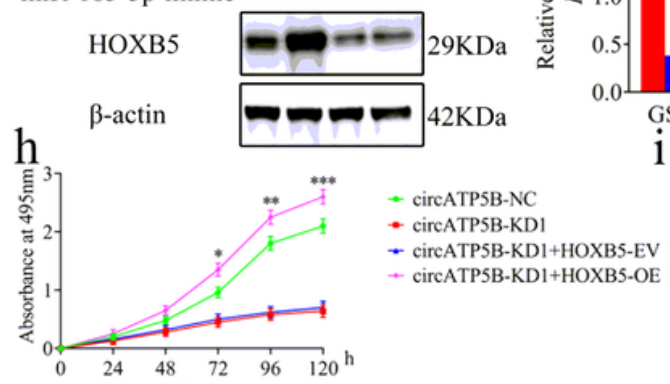

j

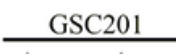

HOXB5-EY

KD1
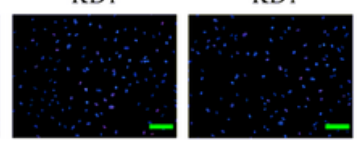

HOXB5-NC

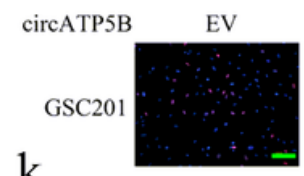

$\mathrm{k}$

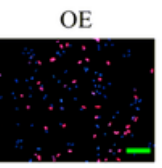

circATP5B

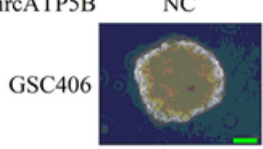

KDI

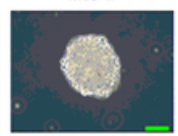

circATP5B

EV

GSC201
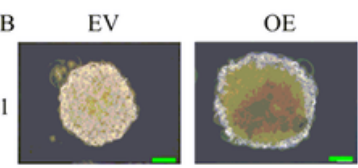

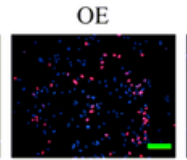

HOXB5-EV

KD1

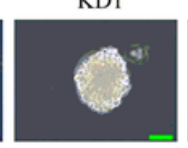

HOXB5-NC

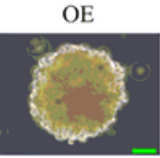

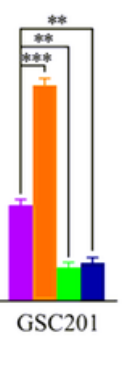

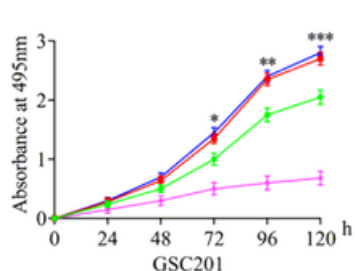

- circATP5B-EV

- circATP5B-OE+HOXB5-NC

- circATP5B-OE+HOXB5-KD1

circATP5B-NC+miR-185-5p-NC circATP5B-KDI+miR-185-5p-NC circATP5B-KDl+miR-185-5p-inhibitor circATP5B-EV+miR-185-5p-NC

circATP5B-OE+miR-185-5p-NC

circATP5B-EV+miR-185-5p-mimic

circATP5B-OE+miR-185-5p-mimic
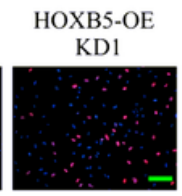

HOXB5-KD1

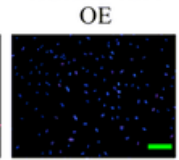

HOXB5-OE

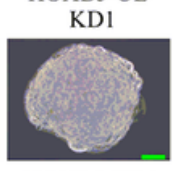

HOXB5-KD1 $\mathrm{OE}$

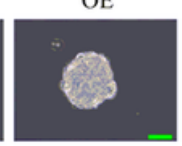

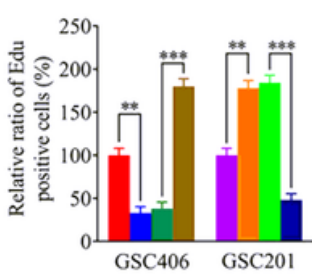

circATP5B-NC

a circATP5B-KDI

circATP5B-KDI+HOXB5-EV

circATP5B-KD1+HOXB5-OE

circATP5B-EV

circATP5B-OE

circATP5B-OE+HOXB5-NC

circATP5B-OE+HOXB5-KD1
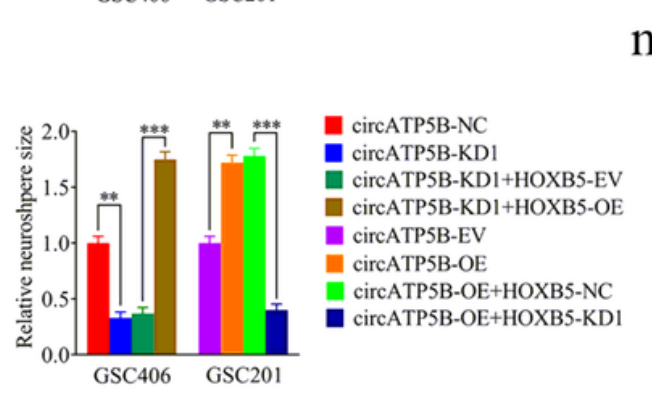

circATP5B-NC

circATP5B-KD1

circATP5B-KD1+HOXB5-EV

circATP5B-KDI+HOXB5-OE

circATP5B-EV

circATP5B-OE

circATP5B-OE+HOXB5-NC

- circATP5B-OE+HOXB5-KD1 $\mathrm{d}_{\text {circATP5B-NC }} \frac{\text { GSC406 }}{+-+}$

circATP5B-KD1 - + - +

miR-185-5p-NC $++-\cdot$

miR-185-5p-inhibitor

HOXB5

$\beta$-actin

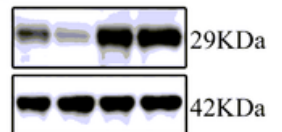

$\mathrm{g}$

$\mathrm{N}$-Total $\mathrm{r}=0.4625 \mathrm{p}=0.0400$

- Grade II r $=0.4840 \mathrm{p}=0.0142$

-Grade III $\mathrm{r}=0.5333 \mathrm{p}=0.006 \%$

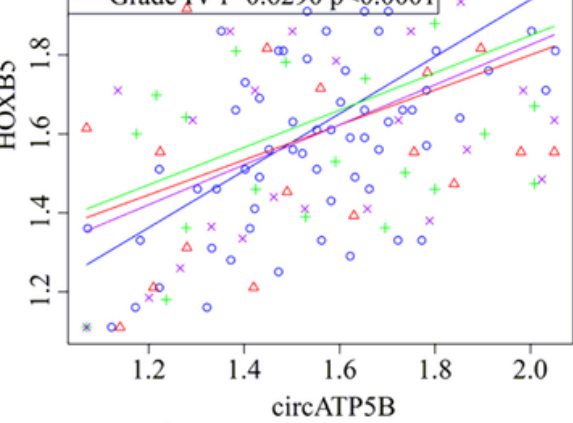

1

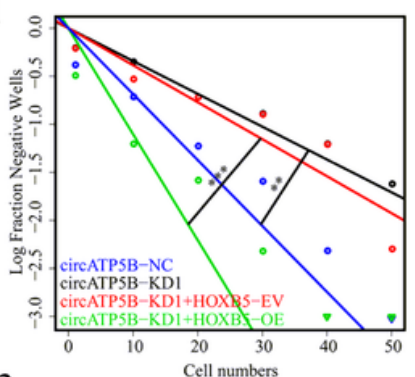

$\mathrm{m}$

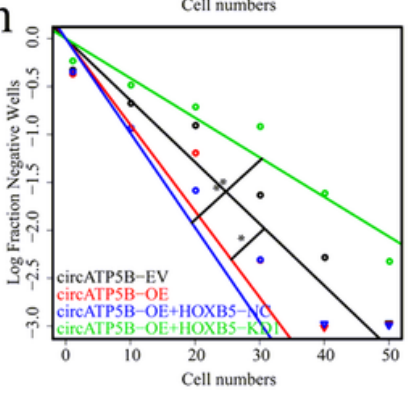

Figure 5

CircATP5B promoted the proliferation of GSCs by upregulating HOXB5 expression. a, b, c qRT-PCR (a) and western blotting $(b, c)$ showed the expression of HOXB5 in GSCs after circATP5B knockdown or overexpression. $d$ The decreased expression of HOXB5 in GSC406 induced by circATP5B knockdown was reversed by miR-185-5p inhibitor treatment, as detected by western blotting. e The increased expression of HOXB5 in GSC201 induced by circATP5B overexpression was reversed by miR-185-5p mimic treatment, as determined by western blotting. $f$ The effects of both circATP5B and miR-185-5p on the expression of HOXB5 in GSCs were detected by qRT-PCR. $\mathrm{g}$ The relative expression correlation between circATP5B and HOXB5 in 70 cases of glioma patients were detected by qRT-PCR. $h$ and i MTS assays showed that circATP5B knockdown or overexpression affected the cell viability of GSCs and was reversed by HOXB5 overexpression or knockdown, respectively. j The EDU assays showed that circATP5B knockdown or 
overexpression affected the proliferation of GSCs and was reversed by HOXB5 overexpression or knockdown, respectively. Scale bar $=50 \mu \mathrm{m}$. $\mathrm{k}$ The neurospheres formation assays showed that circATP5B knockdown or overexpression affected the self-renewing capacity of GSCs and was reversed by HOXB5 overexpression or knockdown, respectively. Scale bar $=20 \mu \mathrm{m}$. I and $\mathrm{m}$ Limiting dilution assays showed that circATP5B knockdown or overexpression affected the tumor formation incidence of GSCs and was reversed by HOXB5 overexpression or knockdown, respectively. All data were expressed as the mean \pm SD (three independent experiments). ${ }^{*} p<0.05 ;{ }^{* *} p<0.01 ;{ }^{* * *} p<0.001$.

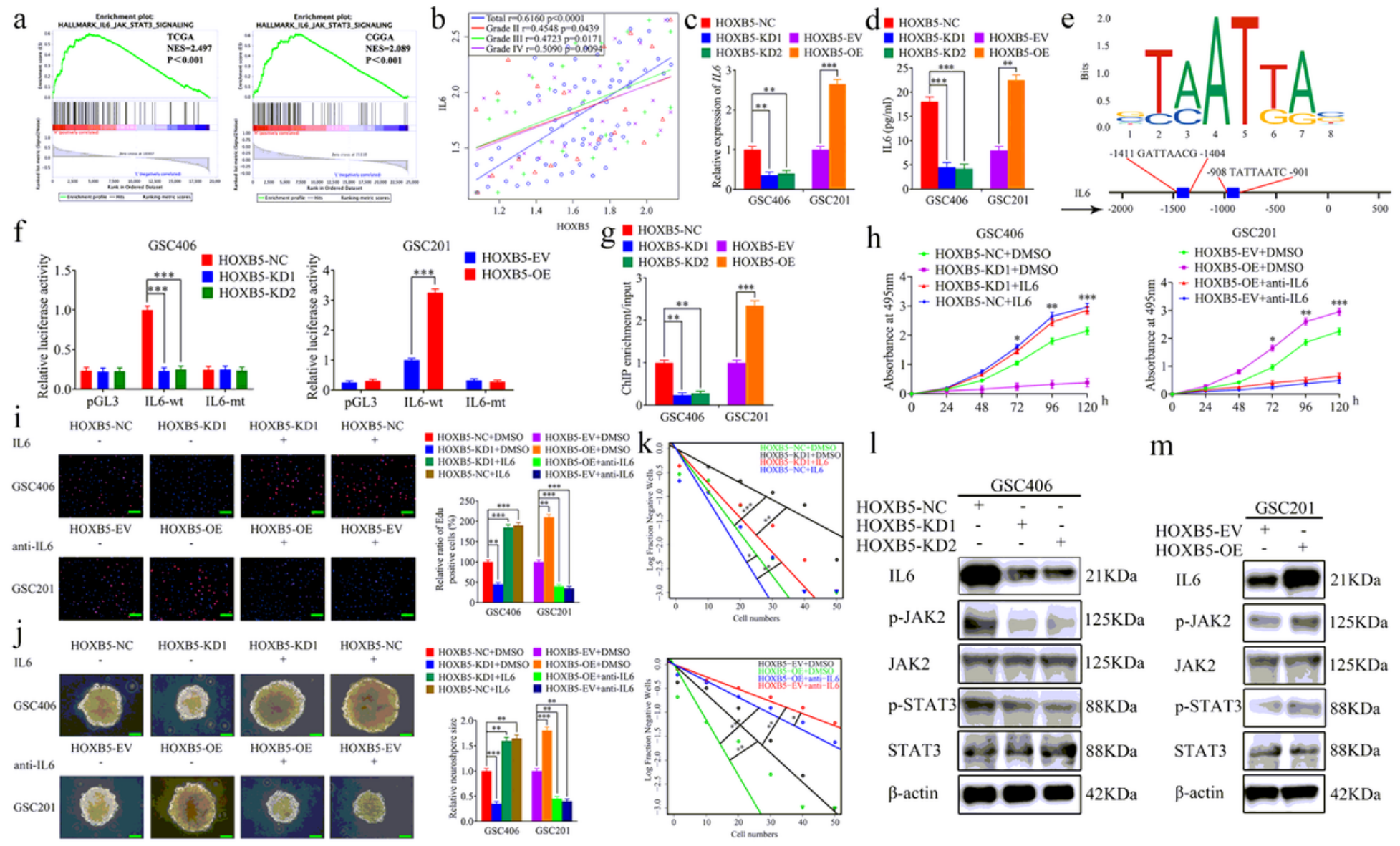

\section{Figure 6}

HOXB5 transcriptionally regulated IL6 expression and regulated the proliferation of GSCs via JAK2/STAT3 signaling. a TCGA and CGGA datasets showed that higher HOXB5 expression was associated with enrichment of IL6-mediated JAK2/STAT3 signaling. $b$ The relative expression correlation between HOXB5 and IL6 in 70 cases of glioma patients was detected by qRT-PCR. c, d, I, m qRT-PCR (b), ELISA (d), and western blotting $(\mathrm{l}, \mathrm{m})$ showed the IL6 expression was altered after HOXB5 knockdown or overexpression in GSCs. e Sequence motif representing the consensus HOXB5 binding motif (JASPAR database), and Schematic diagram of the putative HOXB5 binding site in the 3'-UTR of IL6. $f$ The luciferase reporter assays showed that HOXB5 knockdown or overexpression affected the luciferase activities of IL6 in GSCs. g The ChIP qRT-PCR showed that HOXB5 bound to the promoter of IL6. h MTS assays showed that HOXB5 knockdown or overexpression affected the cell viability of GSCs and was reversed by additional recombinant IL6 or anti-IL6, respectively. i The EDU assays showed that HOXB5 
knockdown or overexpression affected the proliferation of GSCs and was reversed by additional recombinant IL6 or anti-IL6, respectively. Scale bar $=50 \mu \mathrm{m}$. j The neurospheres formation assays showed that HOXB5 knockdown or overexpression affected the self-renewing capacity of GSCs and was reversed by additional recombinant IL6 or anti-IL6, respectively. Scale bar $=20 \mu \mathrm{m}$. $\mathrm{k}$ Limiting dilution assays showed that HOXB5 knockdown or overexpression affected the tumor formation incidence of GSCs and was reversed by additional recombinant IL6 or anti-IL6, respectively. I and $m$ Western blotting showed the expression of downstream targets of the IL6-mediated JAK2/STAT3 signaling pathway with HOXB5 knockdown or overexpression in GSCs. All data were expressed as the mean \pm SD (three independent experiments). ${ }^{\star} p<0.05 ;{ }^{* \star} p<0.01 ; * \star * p<0.001$.

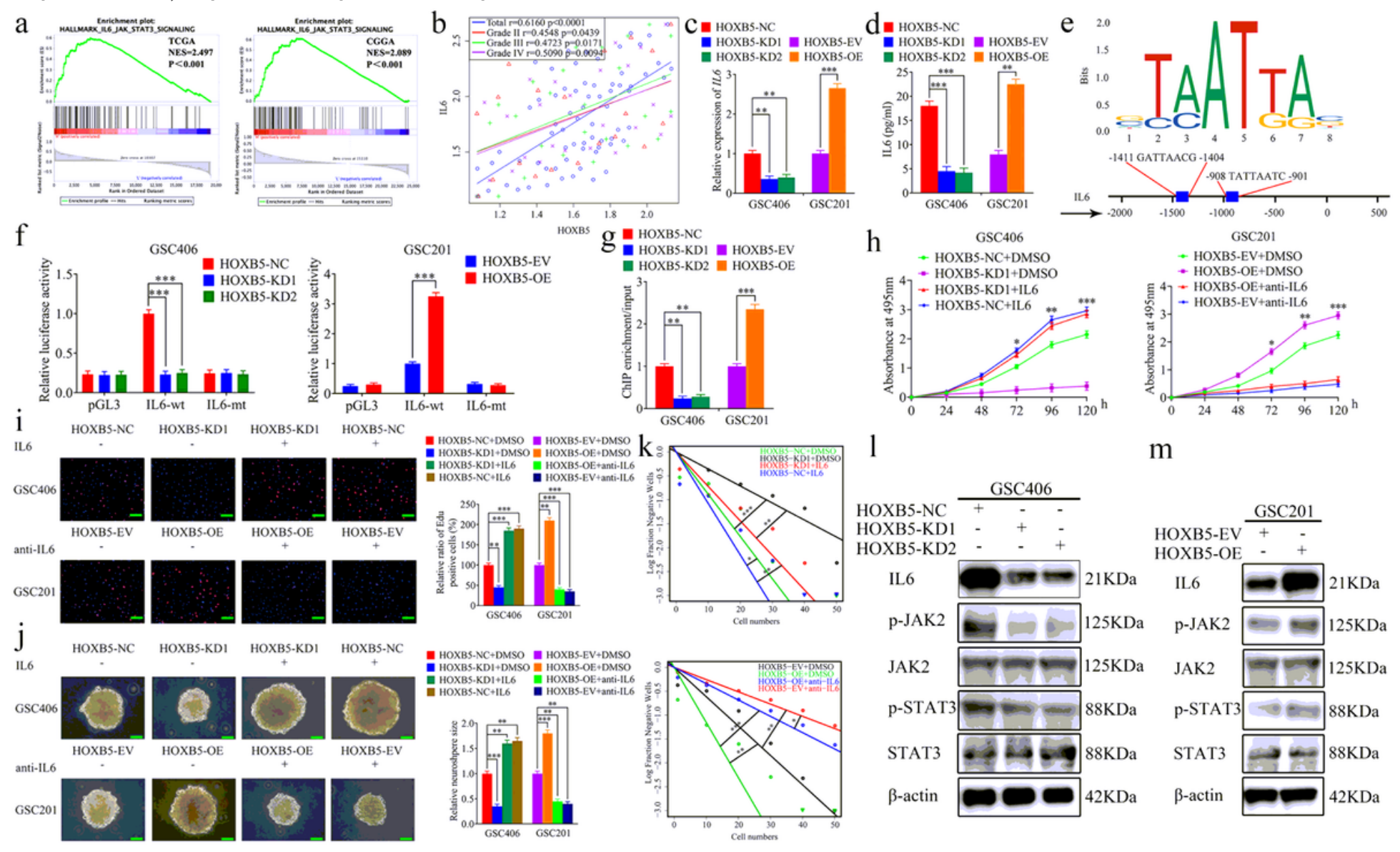

\section{Figure 6}

HOXB5 transcriptionally regulated IL6 expression and regulated the proliferation of GSCs via JAK2/STAT3 signaling. a TCGA and CGGA datasets showed that higher HOXB5 expression was associated with enrichment of IL6-mediated JAK2/STAT3 signaling. b The relative expression correlation between HOXB5 and IL6 in 70 cases of glioma patients was detected by qRT-PCR. c, d, I, m qRT-PCR (b), ELISA (d), and western blotting $(I, m)$ showed the IL6 expression was altered after HOXB5 knockdown or overexpression in GSCs. e Sequence motif representing the consensus HOXB5 binding motif (JASPAR database), and Schematic diagram of the putative HOXB5 binding site in the 3'-UTR of IL6. $f$ The luciferase reporter assays showed that HOXB5 knockdown or overexpression affected the luciferase activities of IL6 in GSCs. g The ChIP qRT-PCR showed that HOXB5 bound to the promoter of IL6. h MTS 
assays showed that HOXB5 knockdown or overexpression affected the cell viability of GSCs and was reversed by additional recombinant IL6 or anti-IL6, respectively. i The EDU assays showed that HOXB5 knockdown or overexpression affected the proliferation of GSCs and was reversed by additional recombinant IL6 or anti-IL6, respectively. Scale bar $=50 \mu \mathrm{m}$. $j$ The neurospheres formation assays showed that HOXB5 knockdown or overexpression affected the self-renewing capacity of GSCs and was reversed by additional recombinant IL6 or anti-IL6, respectively. Scale bar $=20 \mu \mathrm{m}$. $\mathrm{k}$ Limiting dilution assays showed that HOXB5 knockdown or overexpression affected the tumor formation incidence of GSCs and was reversed by additional recombinant IL6 or anti-IL6, respectively. I and $m$ Western blotting showed the expression of downstream targets of the IL6-mediated JAK2/STAT3 signaling pathway with HOXB5 knockdown or overexpression in GSCs. All data were expressed as the mean \pm SD (three independent experiments). ${ }^{\star} p<0.05 ;{ }^{* \star} p<0.01 ; * \star \star x p<0.001$. 

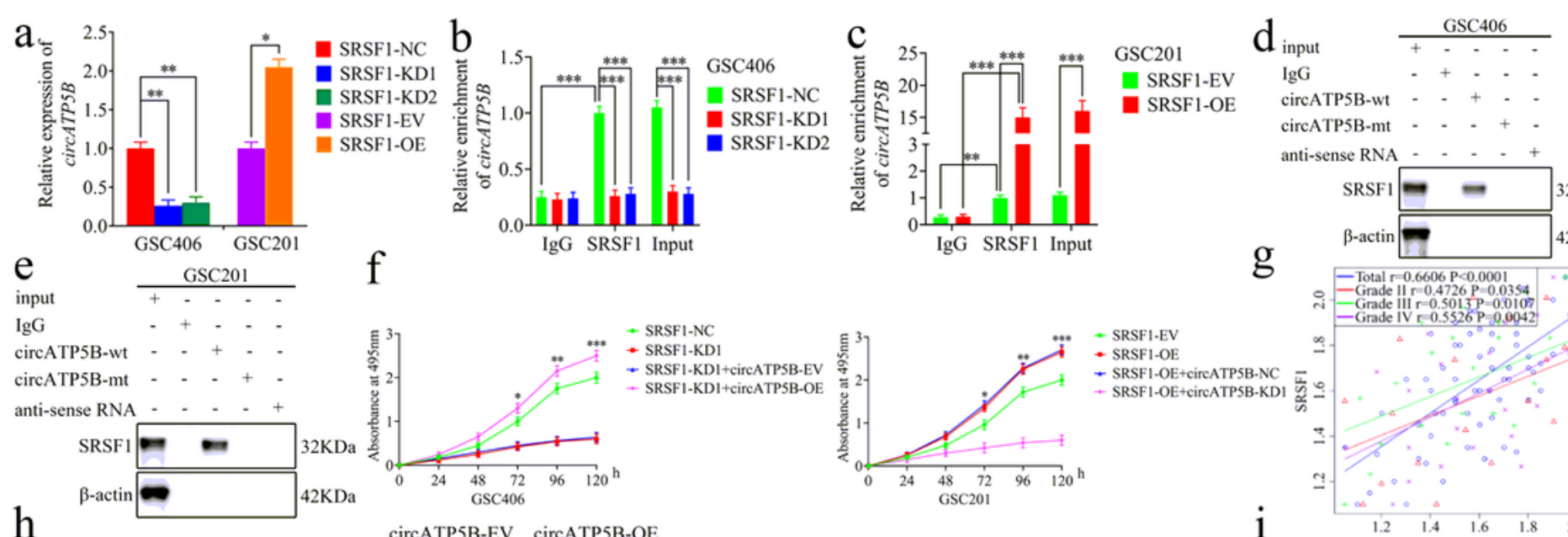

circATP5B-mt - - - +

anti-sense RNA

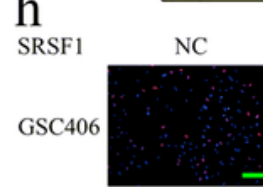
$42 \mathrm{KDa}$

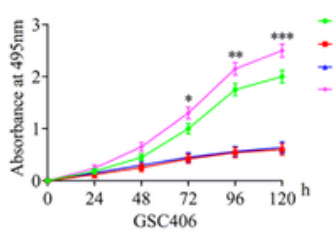

circATP5B-EV circATP5B-OE
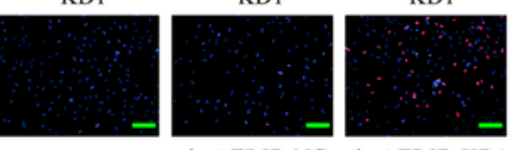

circATP5B-NC circATP5B-KD1

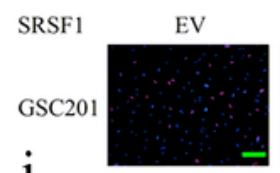

J
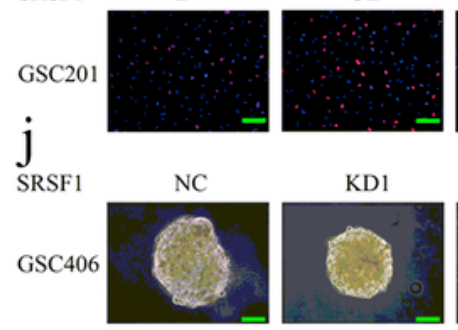

$\mathrm{OE}$

$\mathrm{OE}$

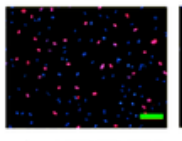

circATP5B-EV circATP5B-OE KD1 KD1

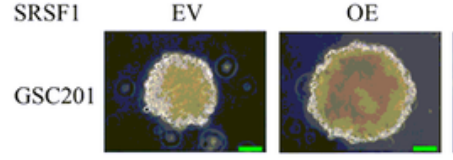

circATP5B-NC circATP5B-KD1

circATPSB-NC circATPSB-KDI
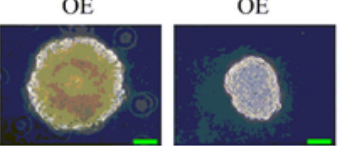

1

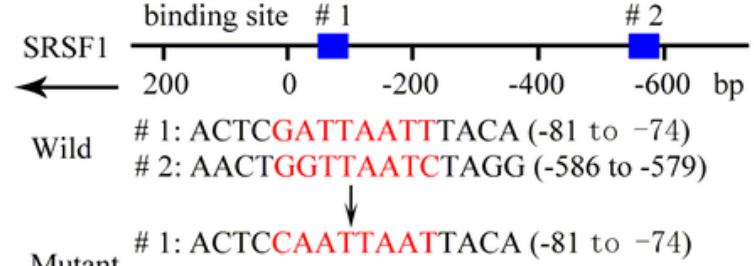

Mutant \# 2: AACTCGATTAACTAGG (-586 to -579$)$
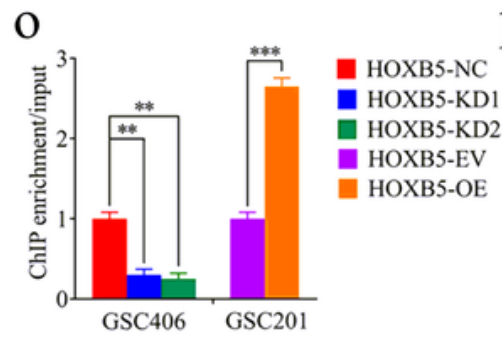

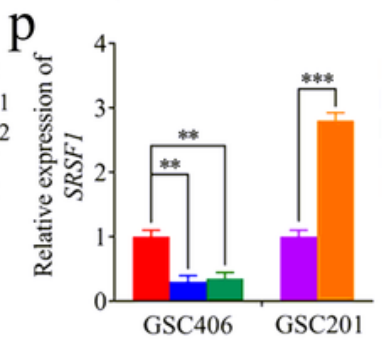

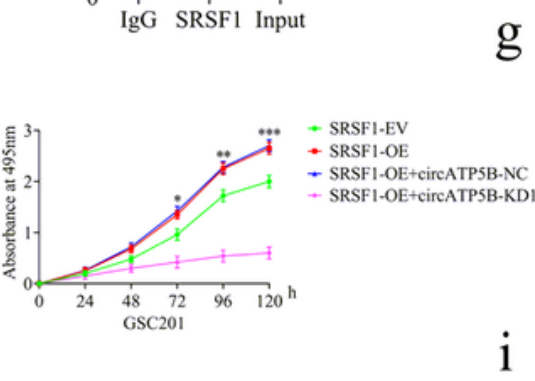

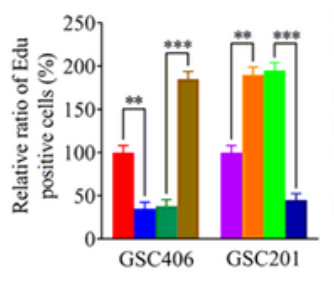

- SRSF1-NC

- SRSFI-KDI

- SRSF1-KD1+circATP5B-EV

SRSF1-KDI+circATP5B-OE

- SRSFI-EV

- SRSF1-OE

SRSF1-OE+circATP5B-NC

- SRSF1-OE+circATP5B-KDI

i

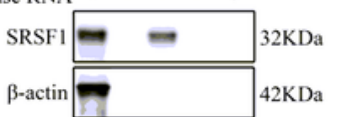

$g$

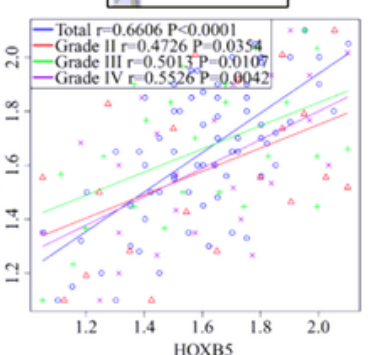

$\mathrm{k}$
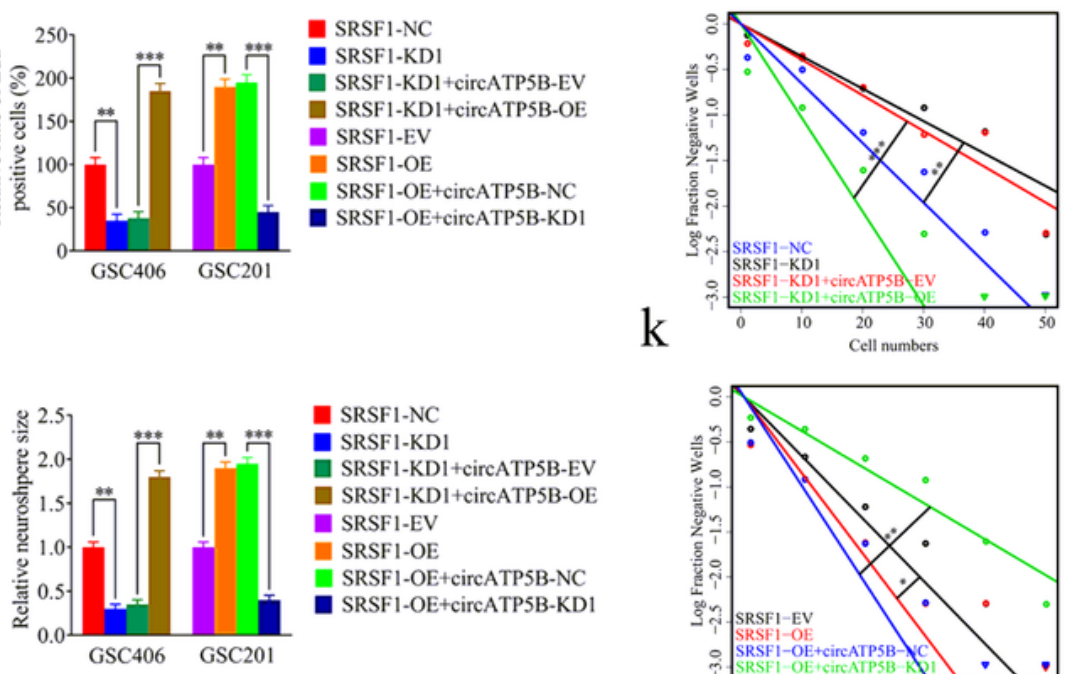

- SRSFI-NC

- SRSF1-KDI

- SRSF1-KD1+circATPSB-EV

SRSF1-KD1+circATP5B-OE

- SRSF1-EV

SRSFI-OE

SRSF1-OE+circATP5B-NC

- SRSF1-OE+circATP5B-KD

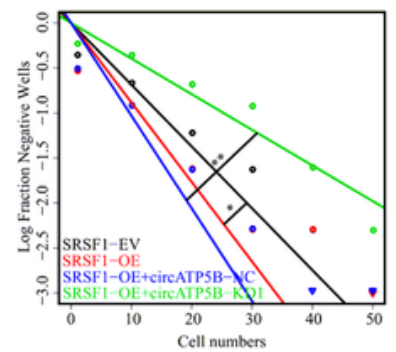

$\mathrm{m}$

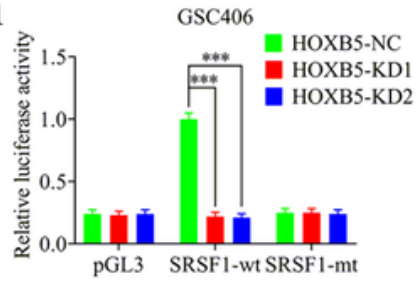

n

q

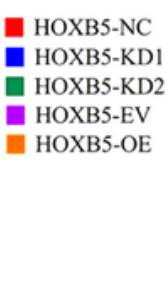

G $\mathrm{GSC} 406$

HOXB5-NC +-
HOXB5-KD1 -

HOXB5-KD2

SRSF1

$\beta$-actin

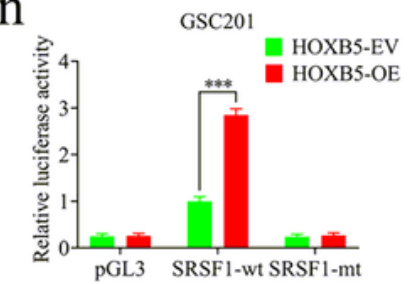

$\mathrm{r}$

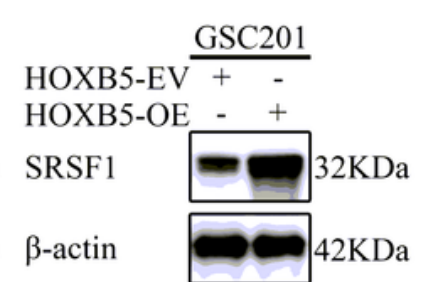

Figure 7

SRSF1 regulated the proliferation of GSCs by binding to circATP5B and upregulating circATP5B expression. a The relative expression of circATP5B after SRSF1 knockdown or overexpression was detected by qRT-PCR. b, c The RIP assay was performed after SRSF1 knockdown (b) or overexpression (c), followed by qRT-PCR to detect the enrichment of circATP5B in GSCs. d, e The RNA pull-down assays showed the SRSF1 protein immunoprecipitation with circATP5B as detected by western blotting. $\mathrm{f}$ MTS 
assays showed that SRSF1 knockdown or overexpression affected the cell viability of GSCs and was reversed by circATP5B overexpression or knockdown, respectively. $g$ The relative expression correlation between HOXB5 and SRSF1 in 70 cases of glioma patients were detected by qRT-PCR. $\mathrm{h}$ The EDU assays showed that SRSF1 knockdown or overexpression affected the proliferation of GSCs and was reversed by circATP5B overexpression or knockdown, respectively. Scale bar $=50 \mu \mathrm{m}$. $j$ The neurospheres formation assays showed that SRSF1 knockdown or overexpression affected the self-renewing capacity of GSCs and was reversed by circATP5B overexpression or knockdown, respectively. Scale bar $=20 \mu \mathrm{m}$. i and k Limiting dilution assays showed that SRSF1 knockdown or overexpression affected the tumor formation incidence of GSCs and was reversed by circATP5B overexpression or knockdown, respectively. I Schematic diagram of the putative HOXB5 binding site in the 3'-UTR of SRSF1. $m$ and $n$ The luciferase reporter assays showed that HOXB5 knockdown or overexpression affected the luciferase activities of SRSF1 in GSCs. o The ChIP qRT-PCR showed that HOXB5 bound to the promoter of SRSF1. p, q, r qRTPCR (p) and western blotting ( $q, r)$ showed the SRSF1 expression was affected after HOXB5 knockdown or overexpression in GSCs. All data were expressed as the mean \pm SD (three independent experiments). ${ }^{*} p<0.05 ;{ }^{* *} p<0.01 ; * \star * p<0.001$. 

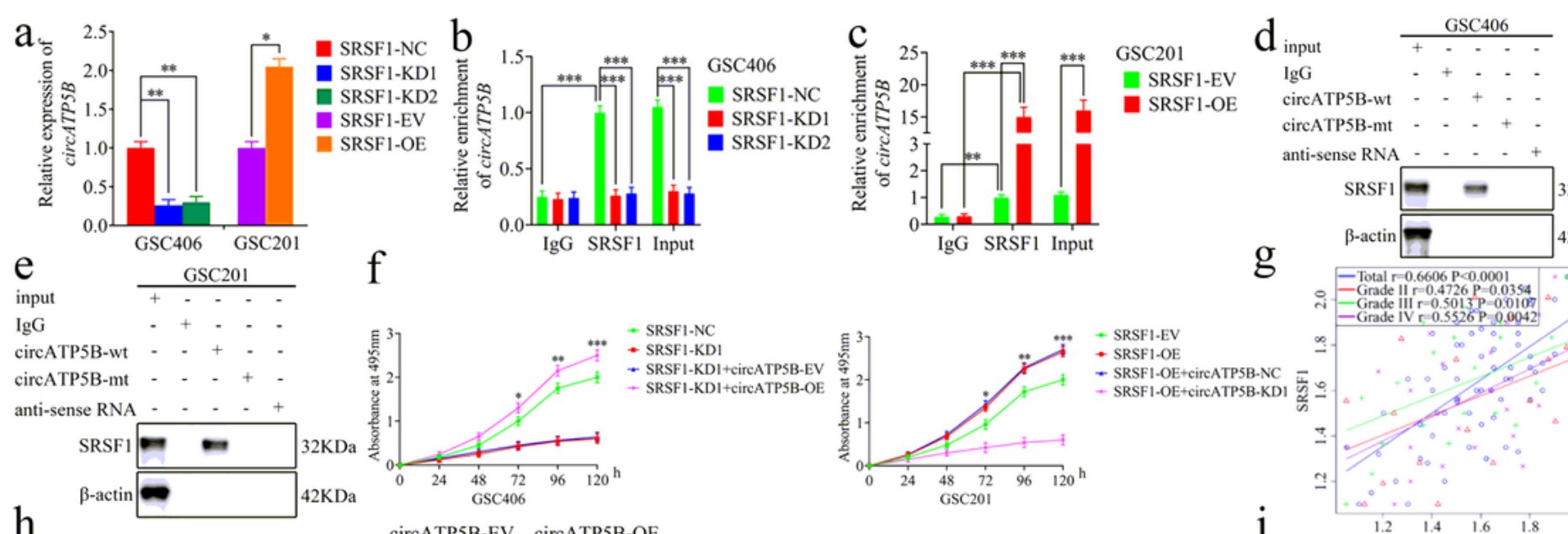

circATP5B-mt - - - + -

anti-sense RNA

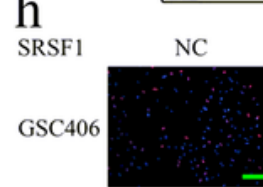
$42 \mathrm{KDa}$

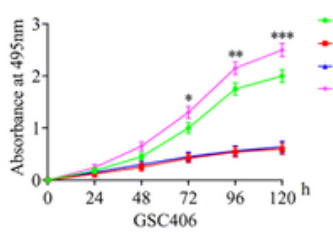

circATP5B-EV circATP5B-OE
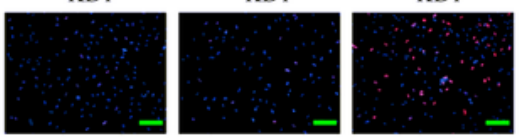

circATP5B-NC circATP5B-KD1

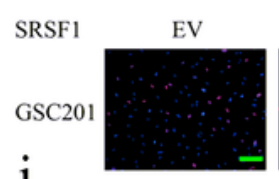

J

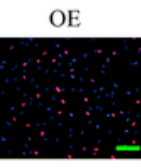
OE

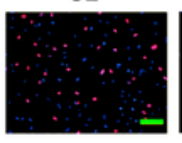

$\mathrm{OE}$

circATP5B-EV circATP5B-OE

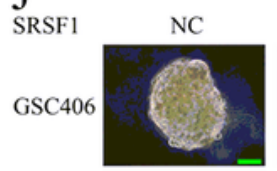

KD1
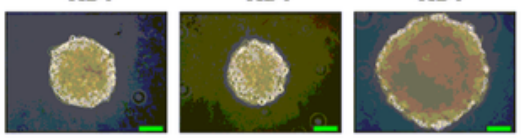

circATP5B-NC circATP5B-KD1
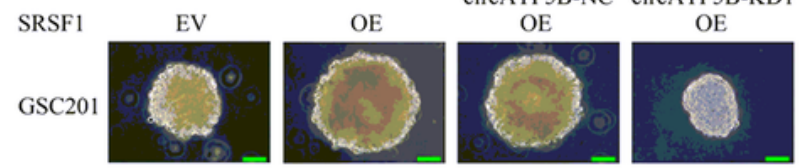

1
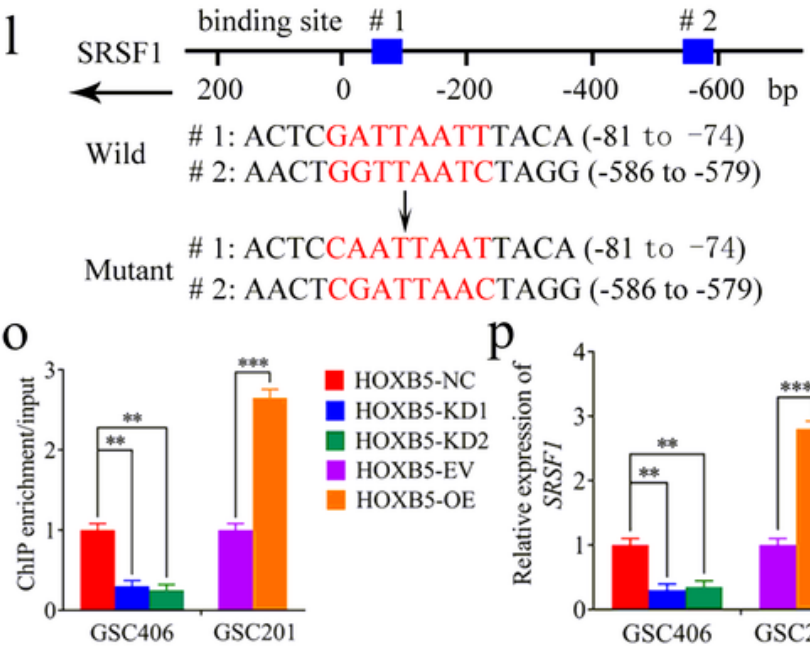

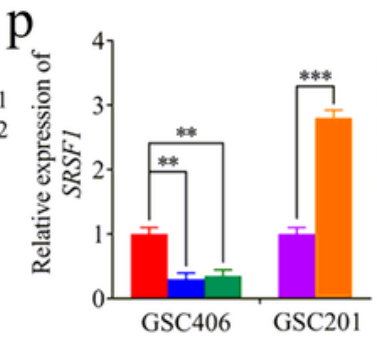

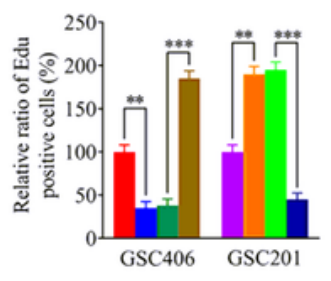

- SRSF1-NC

- SRSFI-KDI

- SRSF1-KD1+circATP5B-EV

SRSF1-KDI+circATP5B-OE

- SRSFI-EV

SRSF1-OE

SRSF1-OE+circATP5B-NC

- SRSF1-OE+circATP5B-KDI

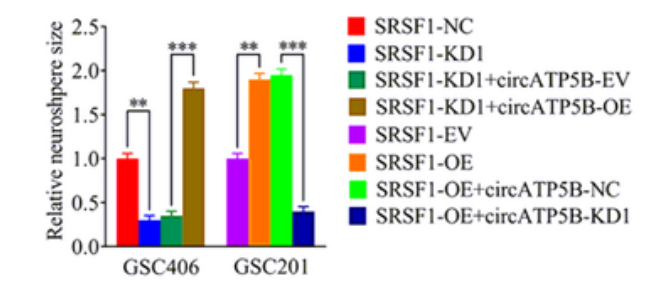

$\mathrm{k}$
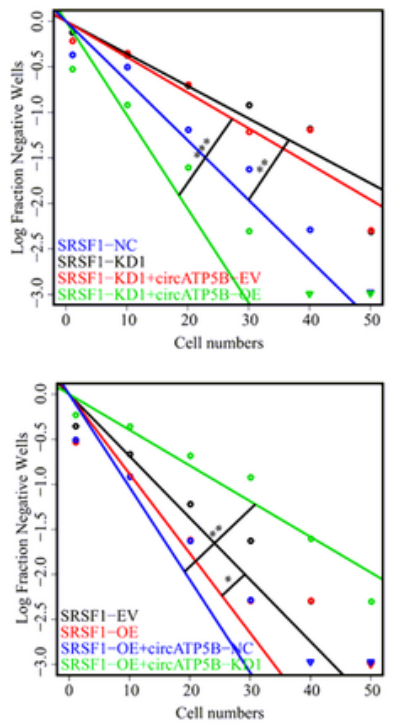

$\mathrm{m}$

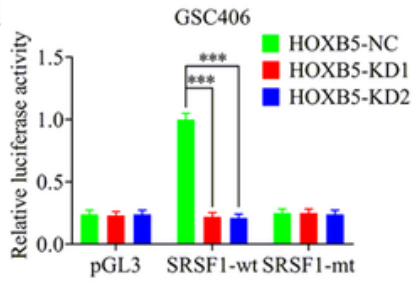

n

q

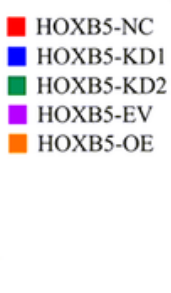

GSC406

HOXB5-NC $+-\cdot$

HOXB5-KD1

HOXB5-KD2

SRSF 1

$\beta$-actin

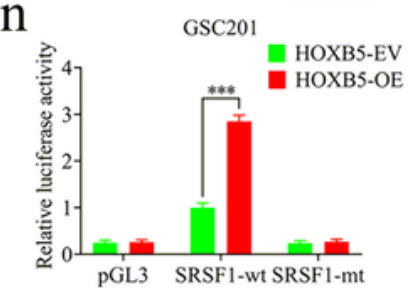

r

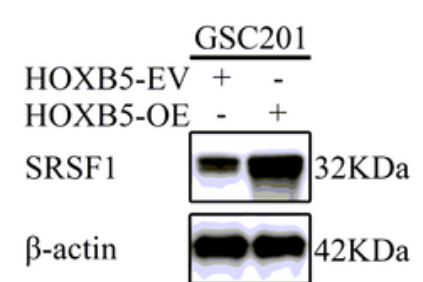

Figure 7

SRSF1 regulated the proliferation of GSCs by binding to circATP5B and upregulating circATP5B expression. a The relative expression of circATP5B after SRSF1 knockdown or overexpression was detected by qRT-PCR. b, c The RIP assay was performed after SRSF1 knockdown (b) or overexpression (c), followed by qRT-PCR to detect the enrichment of circATP5B in GSCs. d, e The RNA pull-down assays showed the SRSF1 protein immunoprecipitation with circATP5B as detected by western blotting. $\mathrm{f}$ MTS 
assays showed that SRSF1 knockdown or overexpression affected the cell viability of GSCs and was reversed by circATP5B overexpression or knockdown, respectively. $g$ The relative expression correlation between HOXB5 and SRSF1 in 70 cases of glioma patients were detected by qRT-PCR. $\mathrm{h}$ The EDU assays showed that SRSF1 knockdown or overexpression affected the proliferation of GSCs and was reversed by circATP5B overexpression or knockdown, respectively. Scale bar $=50 \mu \mathrm{m}$. $j$ The neurospheres formation assays showed that SRSF1 knockdown or overexpression affected the self-renewing capacity of GSCs and was reversed by circATP5B overexpression or knockdown, respectively. Scale bar $=20 \mu \mathrm{m}$. i and k Limiting dilution assays showed that SRSF1 knockdown or overexpression affected the tumor formation incidence of GSCs and was reversed by circATP5B overexpression or knockdown, respectively. I Schematic diagram of the putative HOXB5 binding site in the 3'-UTR of SRSF1. $m$ and $n$ The luciferase reporter assays showed that HOXB5 knockdown or overexpression affected the luciferase activities of SRSF1 in GSCs. o The ChIP qRT-PCR showed that HOXB5 bound to the promoter of SRSF1. p, q, r qRTPCR (p) and western blotting ( $q, r)$ showed the SRSF1 expression was affected after HOXB5 knockdown or overexpression in GSCs. All data were expressed as the mean \pm SD (three independent experiments). ${ }^{*} p<0.05 ;{ }^{* *} p<0.01 ; * \star * p<0.001$. 


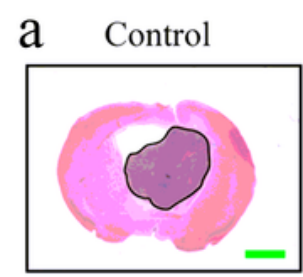

SRSF1-OE
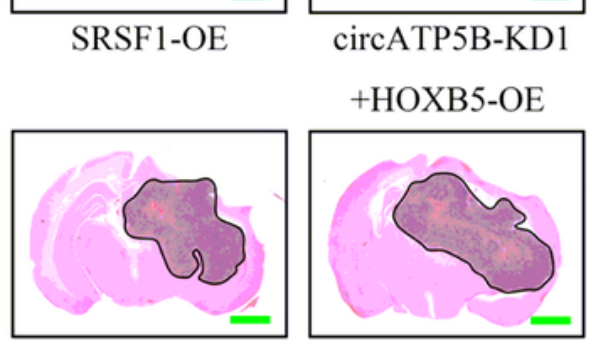
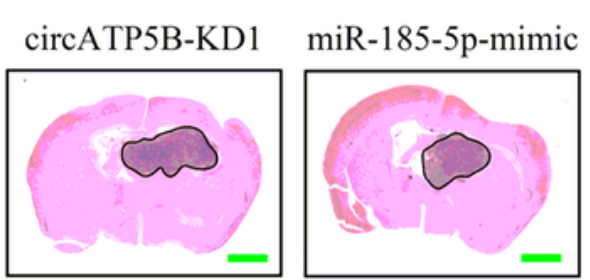

miR-185-5p-mimic

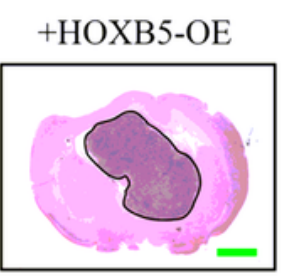

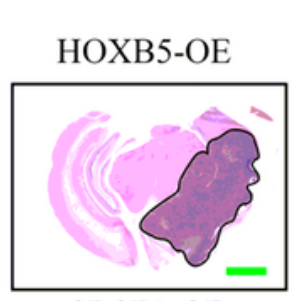

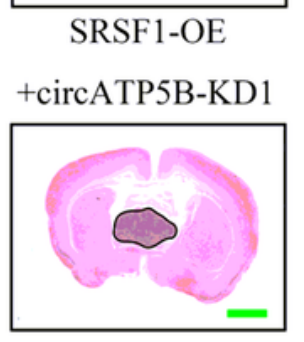

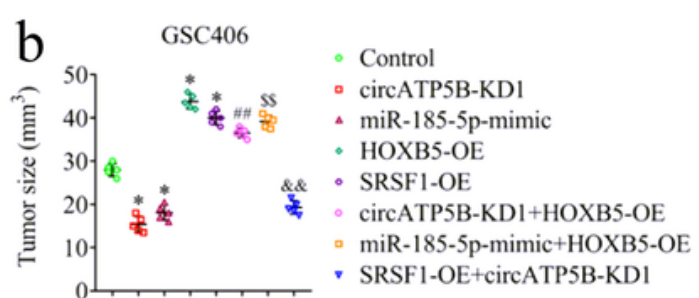

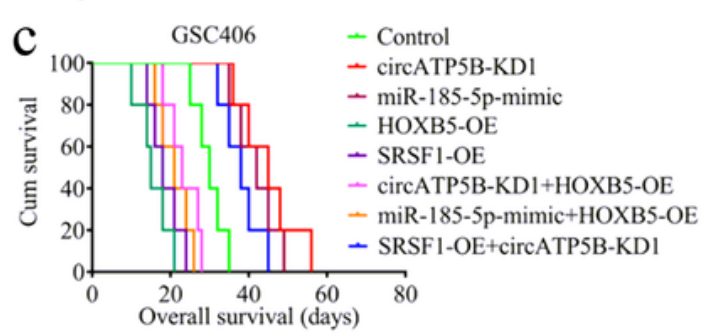

d

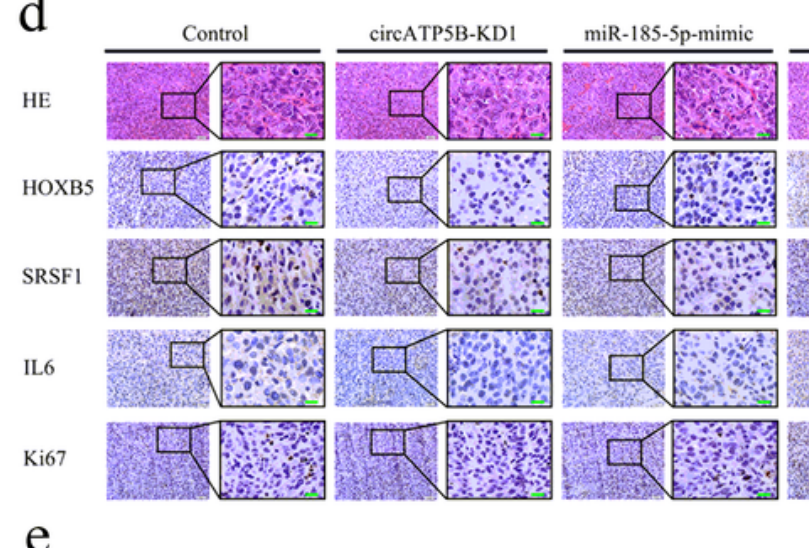

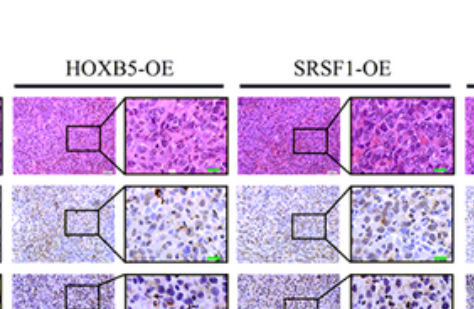

circATP5B-KDI

miR-185-5p-mimic

SRSF1-OE

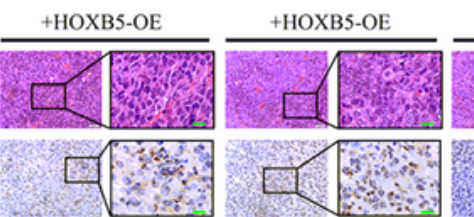
$\frac{+\operatorname{circATP5B}-K D 1}{25{ }^{2}}$ rot
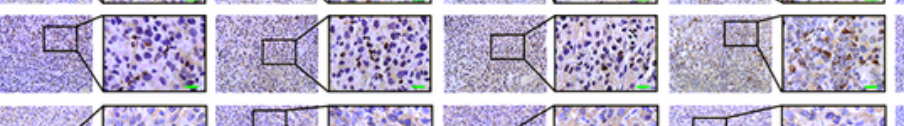

$4 \%$
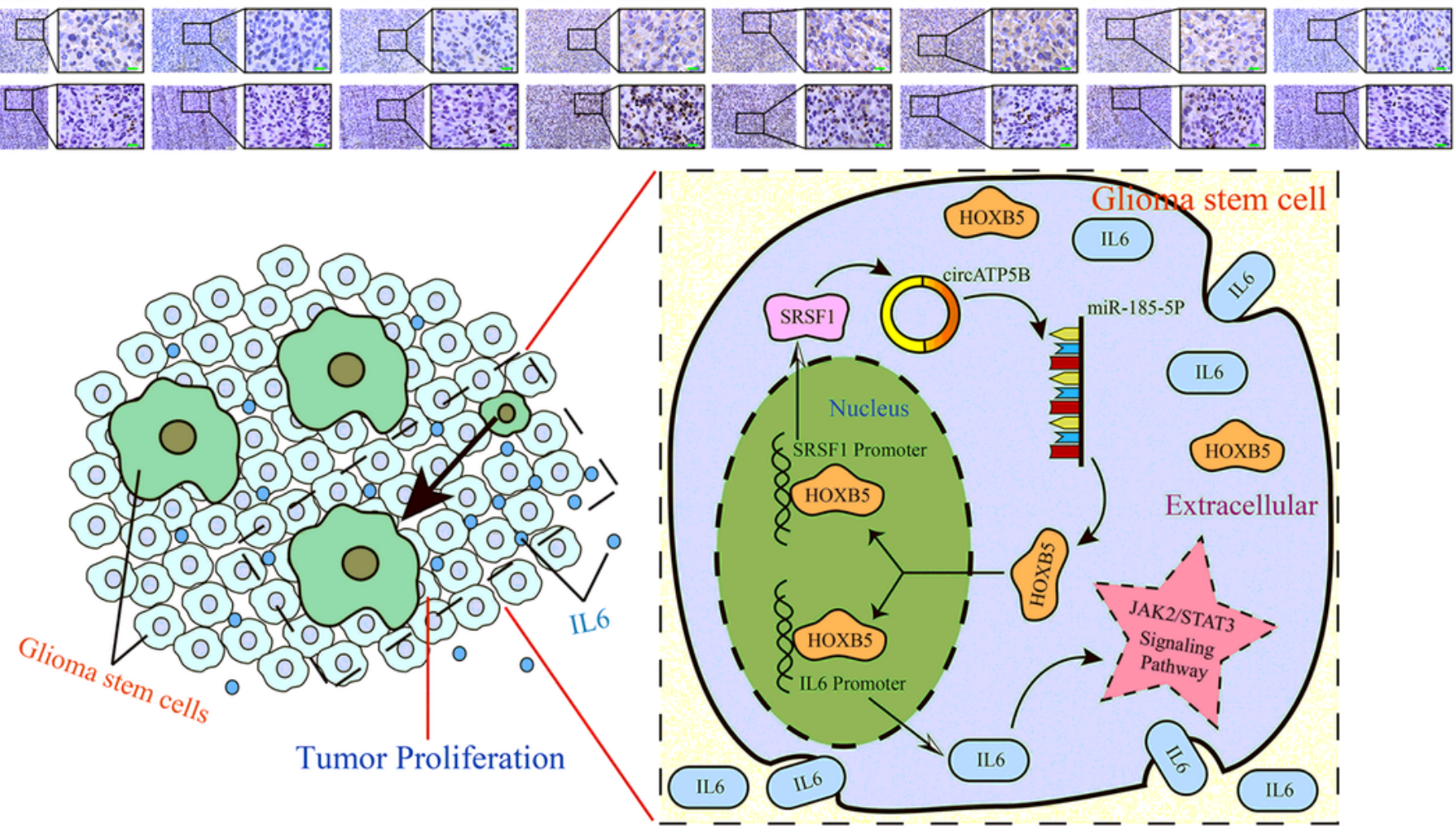

\section{Figure 8}

The SRSF1/circATP5B/miR-185-5p/HOXB5 feedback loop regulated glioma tumorigenesis in vivo. a Representative images showed the size of intracranial tumors in the coronal location of eight groups (negative control, circATP5B knockdown, miR-185-5p mimic, HOXB5 overexpression, SRSF1 overexpression, circATP5B knockdown combined with HOXB5 overexpression, miR-185-5p mimic combined with HOXB5 overexpression, SRSF1 overexpression combined with circATP5B knockdown in 
GSC406). Scale bar $=10 \mathrm{~mm}$. $\mathrm{b}$ The measured tumor volumes among eight GSC406 groups are indicated. c Kaplan-Meier survival curves showed that HOXB5 overexpression, SRSF1 overexpression, circATP5B knockdown combined with HOXB5 overexpression, miR-185-5p mimic combined with HOXB5 overexpression in GSC406 shortened the survival times of nude mice. At the same time, it prolonged the survival times after miR-185-5p mimic was transfected, circATP5B knockdown, and SRSF1 overexpression combined with circATP5B knockdown in GSC406. For each group, $n=5$. $d$ Representative immunohistochemical staining showing the changes in HOXB5, SRSF1, IL6, and Ki67 in the negative control, circATP5B knockdown, miR-185-5p mimic, HOXB5 overexpression, SRSF1 overexpression, circATP5B knockdown combined with HOXB5 overexpression, miR-185-5p mimic combined with HOXB5 overexpression, SRSF1 overexpression combined with circATP5B knockdown group in orthotopic xenograft models. Scale bar $=50 \mu \mathrm{m}$. e Schematic diagram showing that the SRSF1/circATP5B/miR-1855p/HOXB5 axis promoted glioma proliferation through IL6-mediated JAK2/STAT3 signaling pathway. *p< 0.05 vs. the negative control group; \#\#p< 0.05 vs. the circATP5B knockdown group, $\$ \$ p<0.05$ vs. the miR$185-5 p$ group, $\& \& p<0.05$ vs. the SRSF1 overexpression group. All data were expressed as the mean \pm SD (three independent experiments). 


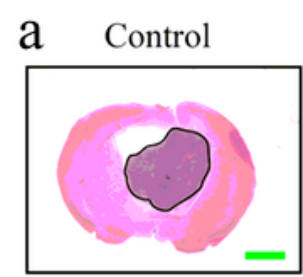

SRSF1-OE

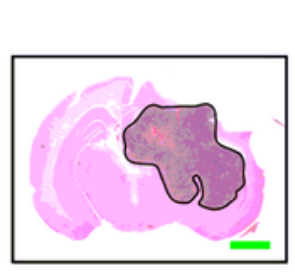

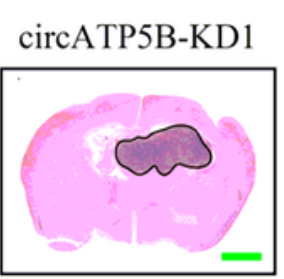

circATP5B-KD1
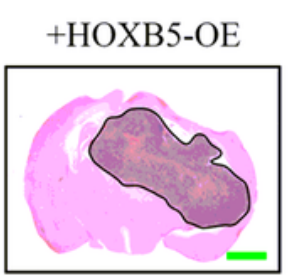

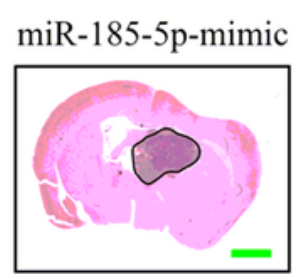

miR-185-5p-mimic

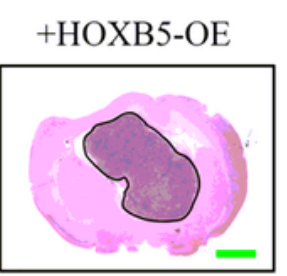

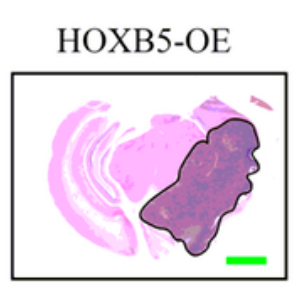

SRSF1-OE

+ circATP5B-KD1

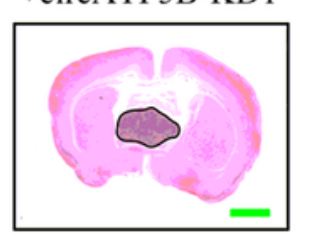

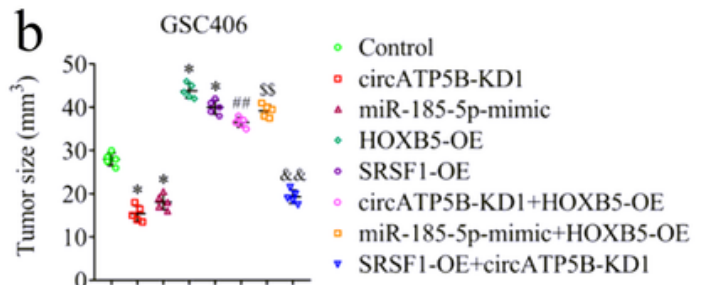

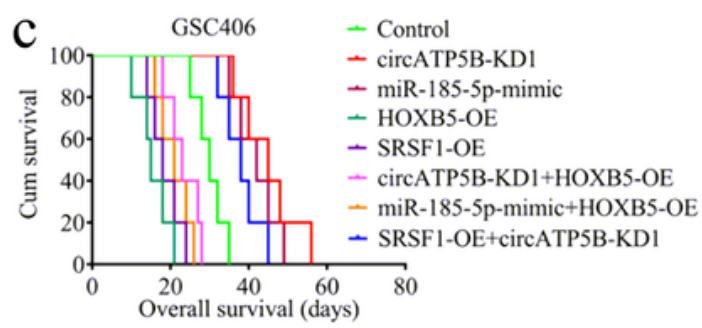

d

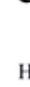

H

HOXB5

SRSFI

IL6

Ki67

e

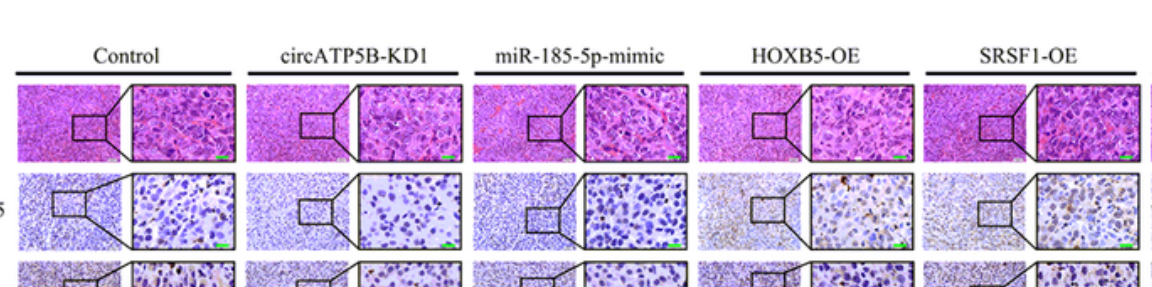

circATP5B-KD1 miR-185-5p-mimic

SRSF1-OE

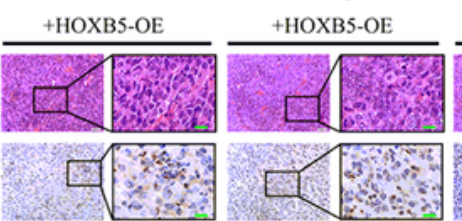

$\frac{+ \text { circATP5B-KD1 }}{\text { का }}$

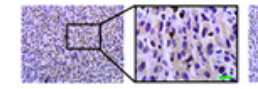

$5 \sqrt{3}$

W.

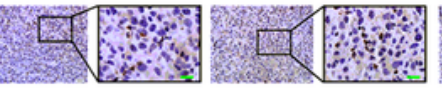

5.

प्रा:

$5 \times$

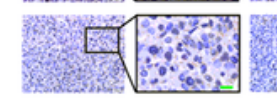

C.

이는

디일

팔.

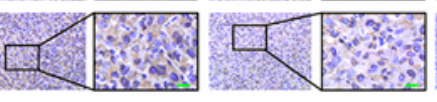

$\square$
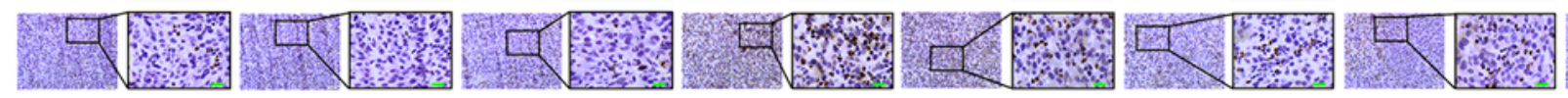

पㄹ.:

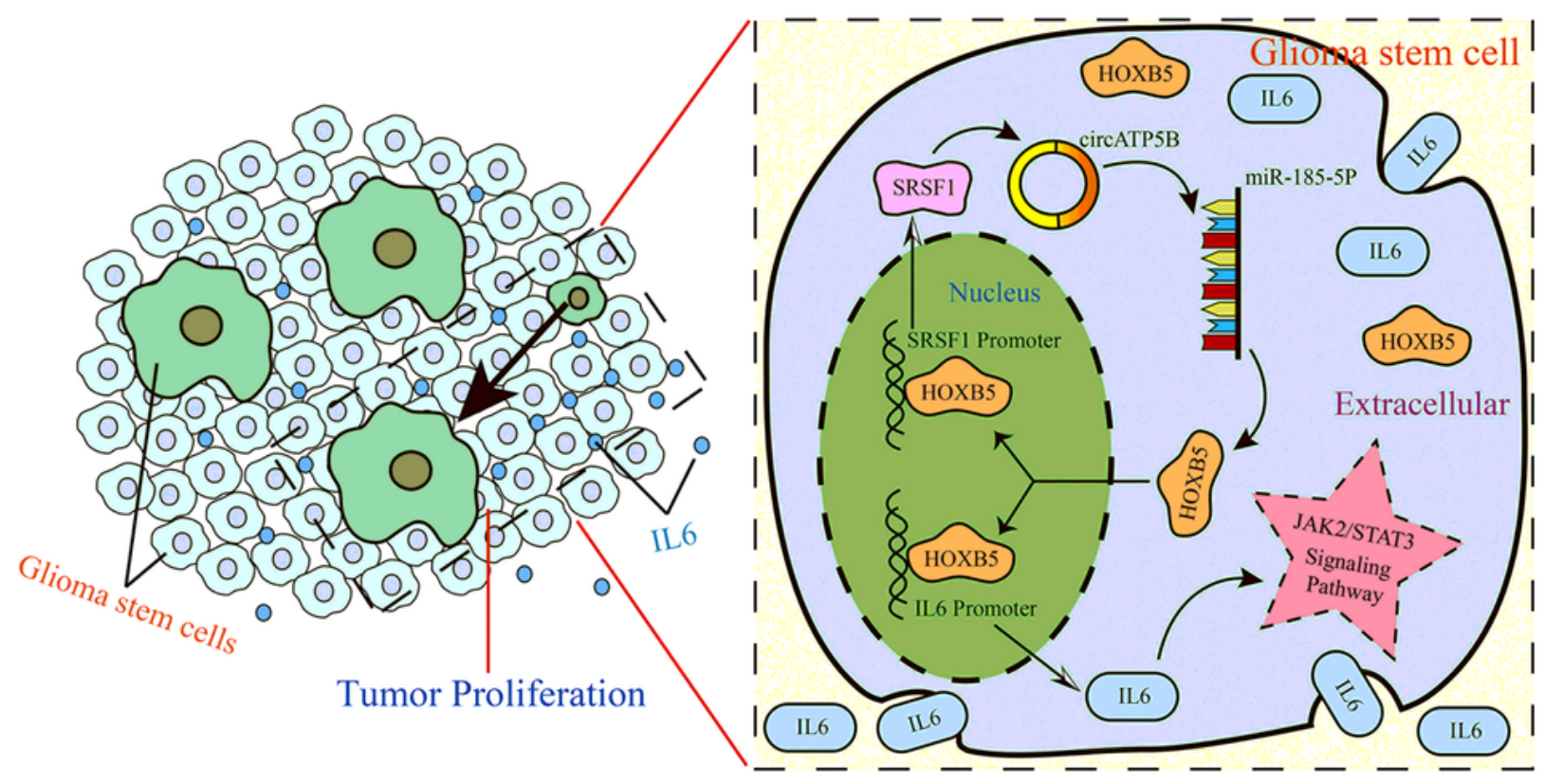

\section{Figure 8}

The SRSF1/circATP5B/miR-185-5p/HOXB5 feedback loop regulated glioma tumorigenesis in vivo. a Representative images showed the size of intracranial tumors in the coronal location of eight groups (negative control, circATP5B knockdown, miR-185-5p mimic, HOXB5 overexpression, SRSF1 overexpression, circATP5B knockdown combined with HOXB5 overexpression, miR-185-5p mimic combined with HOXB5 overexpression, SRSF1 overexpression combined with circATP5B knockdown in 
GSC406). Scale bar $=10 \mathrm{~mm}$. $\mathrm{b}$ The measured tumor volumes among eight GSC406 groups are indicated. c Kaplan-Meier survival curves showed that HOXB5 overexpression, SRSF1 overexpression, circATP5B knockdown combined with HOXB5 overexpression, miR-185-5p mimic combined with HOXB5 overexpression in GSC406 shortened the survival times of nude mice. At the same time, it prolonged the survival times after miR-185-5p mimic was transfected, circATP5B knockdown, and SRSF1 overexpression combined with circATP5B knockdown in GSC406. For each group, $n=5$. $d$ Representative immunohistochemical staining showing the changes in HOXB5, SRSF1, IL6, and Ki67 in the negative control, circATP5B knockdown, miR-185-5p mimic, HOXB5 overexpression, SRSF1 overexpression, circATP5B knockdown combined with HOXB5 overexpression, miR-185-5p mimic combined with HOXB5 overexpression, SRSF1 overexpression combined with circATP5B knockdown group in orthotopic xenograft models. Scale bar $=50 \mu \mathrm{m}$. e Schematic diagram showing that the SRSF1/circATP5B/miR-1855p/HOXB5 axis promoted glioma proliferation through IL6-mediated JAK2/STAT3 signaling pathway. *p< 0.05 vs. the negative control group; \#\#p<0.05 vs. the circATP5B knockdown group, $\$ \$ p<0.05$ vs. the miR$185-5 p$ group, $\& \& p<0.05$ vs. the SRSF1 overexpression group. All data were expressed as the mean \pm SD (three independent experiments).

\section{Supplementary Files}

This is a list of supplementary files associated with this preprint. Click to download.

- SupplementaryFigure1.tif

- SupplementaryFigure1.tif

- SupplementaryFigure2.tif

- SupplementaryFigure2.tif

- SupplementaryTable1.docx

- SupplementaryTable1.docx

- SupplementaryTable2.docx

- SupplementaryTable2.docx

- SupplementaryTable3.docx

- SupplementaryTable3.docx

- SupplementaryTable4.docx

- SupplementaryTable4.docx 